\author{
Universidade de São Paulo \\ Instituto de Física
}

\title{
O DÍLATON EM TEORIAS QUIVER DE HIERARQUIA COMPLETA
}

\author{
Victor Manuel Peralta Cano
}

Dissertação apresentada ao Instituto de Física para a obtenção do título de Mestre em Ciências

Orientador:

Prof. Dr. Gustavo Alberto Burdman (IF/USP)

Banca Examinadora:

Prof. Dr. Gustavo Alberto Burdman (IF/USP)

Prof. Dr. Marcelo Otavio Caminha Gomes (IF/USP)

Prof. Dr. Vicente Pleitez (IFT/UNESP) 



\section{Resumo}

O Modelo Padrão das partículas elementares descreve com sucesso as interações eletrofracas e fortes da natureza, quando comparado com todos os dados experimentais que temos até hoje. Porém, ele apresenta problemas relacionados a origem da quebra da simetria eletrofraca assim como da hierarquia das massas dos férmions. A solução de ambos esses problemas requer a geração de grandes hierarquias estáveis. Essas hierarquias podem ser obtidas em uma classe de teorias quadridimensionais chamadas de teorias quiver de hierarquia completa, que são relacionadas a teorias de dimensões extras em AdS no limite de grande número de sítios. Mostramos que, assim como em teorias de dimensões extras curvas, existe um grau de liberdade leve associado com a quebra da invariância de escala, que pode ser identificado com um dílaton. Partindo da teoria extra-dimensional em um fundo, mostramos como esse dílaton leve também pode ser obtido em teorias quiver de hierarquia completa. 


\section{Abstract}

The standard model of particle physics successfuly describes the electroweak and strong interactions when compared with all the experimental data we have until now. However, it has problems regarding the origin of electroweak symmetry breaking as well as the hierarchy of fermion masses. The solutions of both these problems require the generation of large stable hierarchies. These can be obtained in a class of four-dimensional quiver theories called full-hierarchy quiver theories, which are related to extra dimensional theories in AdS, in the large-number-ofsites limit. We show that, just as in curved extra dimensional theories, there is a light degree of freedom associated with the breaking of scale invariance, which can be identified with a dilaton. Starting from an extra dimensional theory in an $A d S_{5}$ background, we show how this light dilaton can be obtained in full-hierarchy quiver theories as well. 


\section{Agradecimentos}

Gostaria de agradecer aos meus pais, Sebastián Peralta Martinez e Cirila Cano Camargo, não só me ensinaram muito da vida se não também me apóiam nas minhas escolhas da vida.

Quero agradecer em especial ao meu professor e orientador Gustavo A. Burdman, que sempre me motivou no presente trabalho, quem atenciosamente atendeu minhas dúvidas e assim mesmo teve a paciência para meu entendimento do projeto.

Em particular agradecer aos meus colegas, amigos em especial Douglas Mancini, Leila Lobato, Carlos Haluch, Leonardo de Lima, Nayara Fonseca de Sá, Yuber Pérez, Denis Robertson. Muito em especial a Nadia Palomino Buleje

Finalmente, gostaria de agradecer à CAPES pelo apoio financeiro. 


\section{Sumário}

Resumo i

Abstract

Agradecimentos $\quad$ v

1 Introdução 1

1.1 Modelo Padrão . . . . . . . . . . . . . . . . . . . . . . . 1

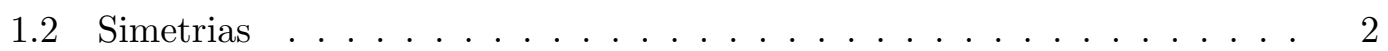

1.2.1 Simetrias Discretas . . . . . . . . . . . . . . . . 2

1.2 .2 Simetria Continua . . . . . . . . . . . . . . . . . 3

1.2 .3 Simetria de Gauge . . . . . . . . . . . . . . . . . . . 8

1.3 Cromodinâmica Quântica (QCD) . . . . . . . . . . . . . . 11

1.4 Modelo eletrofraco . . . . . . . . . . . . . . . . . . . . . 12

1.5 Motivação da Física Além do Modelo Padrão . . . . . . . . . . . . . . . 19

1.5.1 Problema da Hierarquia de Gauge . . . . . . . . . . . . . . . 20

1.5.2 Problema da Hierarquia da Massa dos férmions . . . . . . . . . . 21

1.5.3 Outros Problemas . . . . . . . . . . . . . . . . . . 22

2 Teorias Quiver a partir de Teorias com uma Dimensão Extra $\quad 25$

2.1 Teorias $\mathrm{AdS}_{5}$ com métrica de RS com campos na Bulk 5D . . . . . . . 27

2.1 .1 Bósons de gauge na bulk . . . . . . . . . . . . . . . 29

2.1 .2 Férmions na bulk . . . . . . . . . . . . . . . . . . 33

2.2 Desconstrução de uma Teoria de Gauge . . . . . . . . . . . . . . . . 37

2.3 Desconstrução de uma Teoria que inclui Férmions . . . . . . . . . . . 48

3 Estabilização da Dimensão Extra em $\mathrm{AdS}_{5} \quad 57$

3.1 Mecanismo de Goldberger-Wise . . . . . . . . . . . . . . . . . 59

4 O Dílaton em Teorias Quiver $\quad 65$

4.1 Radion . . . . . . . . . . . . . . . . . . 65

4.2 Estabilização entre as escalas UV e IR . . . . . . . . . . . . . . 71 
5 Conclusão

Apêndices

79

Referências Bibliográficas

90 


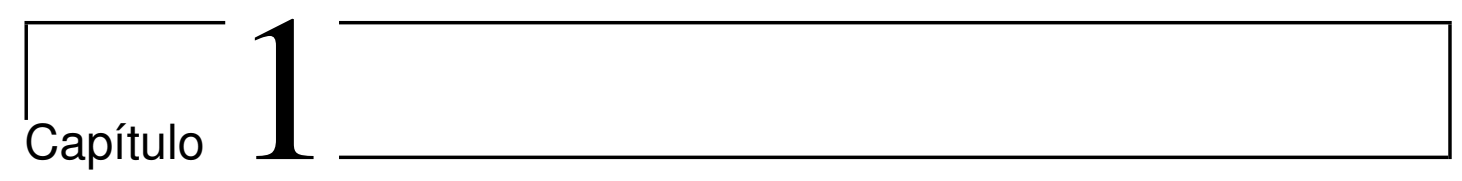

\section{Introdução}

\subsection{Modelo Padrão}

O Modelo Padrão da física de partículas elementares é uma teoria quântica de campos que descreve as interações eletromagnéticas, fracas e fortes. As pesquisas teóricas e experimentais de partículas elementares nos anos 60 deram indícios de uma possível unificação das interações fracas e eletromagnéticas, devido ao fato de ambas serem de caráter vetorial e possuírem acoplamentos universais; em outras palavras, elas tinham como característica serem descritas por uma teoria de gauge. Enfim, entre os anos 60 e 70, o Modelo Padrão foi desenvolvido inicialmente por Glashow, Weinberg e Salam, tornando mais claro o conhecimento das partículas elementares.

Os quatro ingredientes básicos necessários para o Modelo Padrão são as partículas elementares: léptons, quarks, bósons de gauge e o bóson de Higgs. Todas as interações eletrofracas e fortes são explicadas pelas teorias de gauge, isto é a lagrangiana é invariante sob as transformações de gauge de $S U(3)_{C}$ para as interações fortes e é invariante sob as transformações de gauge de $S U(2)_{L} \times U(1)_{Y}$ para as interações eletrofracas. Sendo assim, podemos estudar as interações fortes separado das interações eletrofracas, pois ambos setores de gauge não se misturam. O Modelo Padrão tem um domínio de aplicabilidade de até várias centenas de $\mathrm{GeV}$, cabe mencionar que este tem uma estrutura teórica que trabalha esplendidamente, além de ter dados experimentais bem sucedidos. Além disso, o modelo apresenta simetrias importantes na descrição de tais interações. Em geral, as simetrias tem um papel central na física, isto é, elas protegem alguma quantidade física e determinam a estrutura dinâmica dos campos.

Neste capítulo, além de fazer uma abordagem breve das simetrias, trataremos com ênfase as simetrias de gauge. Em particular aquelas do Modelo Padrão, o qual nos dará as ferramentas básicas para descrever brevemente a QCD. A seguir, usaremos o Mecanismo de Higgs com quebra espontânea da simetria de gauge $S U(2)_{L} \times U(1)_{Y} \rightarrow$ $U(1)_{E M}$ no Modelo eletrofraco ${ }^{1}$, de modo que os bósons vetoriais mediadores da interação fraca e os férmions obtêm massa.

\footnotetext{
${ }^{1} \mathrm{O}$ subgrupo de cor $S U(3)_{C}$ não é quebrado.
} 


\section{$1.2 \quad$ Simetrias}

$\mathrm{Na}$ física se diz que se tem uma simetria se ao mudar as variáveis dinâmicas a ação fica invariante.

\subsubsection{Simetrias Discretas}

Uma simetria é discreta no sentido que matemáticamente ela não pode ser expressada via uma transformação infinitesimal desde a identidade. Vamos falar de algumas simetrias que satisfazem esta condição.

\section{Paridade}

Dentro do grupo de Lorentz temos as transformações imprópias, em particular a transformação de Paridade que é expressada por

$$
\left(x^{0}, \vec{x}\right) \rightarrow x_{P}=\left(x^{0},-\vec{x}\right) .
$$

O efeito sobre um campo do férmion de Dirac é implementado pelo operador unitário $P$

$$
P \psi(x) P^{-1}=\gamma^{0} \psi\left(x_{P}\right) .
$$

\section{Inversão Temporal}

A operação de Inversão Temporal é expressada por

$$
\left(x^{0}, \vec{x}\right) \rightarrow x_{T}=\left(-x^{0}, \vec{x}\right) .
$$

O efeito neste caso sobre um campo fermiônico é implementado pelo operador $T$ antiunitário ${ }^{2}$ é dado por

$$
T \psi(x) T^{-1}=i \gamma^{1} \gamma^{3} \psi\left(x_{T}\right) .
$$

\footnotetext{
${ }^{2} \mathrm{~T}$ é um operador anti linear, isto é $T(c)=(c)^{*} T$, onde c é um número complexo.
} 


\section{Conjugação de Carga}

Vamos considerar uma terceira classe de operação discreta que não é espaço-temporal a qual troca o estado de uma partícula pelo estado de uma antipartícula,

$$
C \psi(x) C^{-1}=i \gamma^{2} \gamma^{0} \bar{\psi}^{T}(x)
$$

onde $\bar{\psi}_{\beta}^{T}=\psi_{\alpha}^{\dagger} \gamma_{\alpha \beta}^{0}(\alpha, \beta=1, \ldots, 4)$. O efeito daz transformações discretas sob os bilineares de Dirac está indicada na tabela de baixo para $\mu=0,1,2,3$.

\begin{tabular}{cccccc}
\hline & $\bar{\psi} \psi$ & $i \bar{\psi} \gamma^{5} \psi$ & $\bar{\psi} \gamma^{\mu} \psi$ & $\bar{\psi} \gamma^{\mu} \gamma^{5} \psi$ & $\bar{\psi} \frac{i}{2}\left[\gamma^{\mu}, \gamma^{\nu}\right] \psi$ \\
\hline$P$ & +1 & -1 & $(-1)^{\mu}$ & $-(-1)^{\mu}$ & $(-1)^{\mu}(-1)^{\nu}$ \\
$T$ & +1 & -1 & $(-1)^{\mu}$ & $(-1)^{\mu}$ & $-(-1)^{\mu}(-1)^{\nu}$ \\
$C$ & +1 & +1 & -1 & +1 & -1 \\
\hline
\end{tabular}

As interações forte e a eletromagnética são simétricas sob $C, P$ e $T$ separadamente [1], mas no setor eletrofraco não todas essas simetrias são satisfeitas.

\subsubsection{Simetria Continua}

Falar de simetria contínua implica em dizer que essas transformações possuem parâmetros contínuos. Se uma ação é invariante sob transformações contínuas, o teorema de Noether nós fornece quantidades que são conservadas associadas a cada transformação. Sabemos que da teoria quântica de campos, se por exemplo temos a translação no espaço-tempo vamos obter que o tensor de energia-momento é uma quantidade conservada. A seguir vamos abordar algumas transformações contínuas. 


\section{Simetria de Isospin}

O sistema núcleon-píon é um meio para estudar a invariância $S U(2)$ da lagrangiana que modela este sistema e calcularemos as correntes conservadas. O campo do núcleon vai ser um dubleto

$$
\psi=\left(\begin{array}{l}
p \\
n
\end{array}\right)
$$

e o campo de píon vai ser um tripleto $\pi=\left\{\pi^{i}\right\},(i=1,2,3)$ a lagrangiana é

$$
\mathcal{L}=\bar{\psi}((i) \not \partial-\boldsymbol{m}) \psi+\frac{1}{2}\left[\partial_{\mu} \boldsymbol{\pi} \cdot \partial^{\mu} \boldsymbol{\pi}-m_{\boldsymbol{\pi}}^{2} \boldsymbol{\pi} \cdot \boldsymbol{\pi}\right]+i g \bar{\psi} \boldsymbol{\tau} \cdot \boldsymbol{\pi} \gamma^{5} \psi-\frac{\lambda}{4}(\boldsymbol{\pi} \cdot \boldsymbol{\pi})^{2}
$$

onde a matriz de massa do núcleon é

$$
\boldsymbol{m}=\left(\begin{array}{cc}
m & 0 \\
0 & m
\end{array}\right),
$$

$\left\{\tau^{i}\right\}$ são as matrizes de Pauli. $\mathcal{L}$ é invariante sob rotação global de $\mathrm{SU}(2)$ dos campos, isto é

$$
\psi \longrightarrow \psi^{\prime}=U \psi, \quad U=\exp (-i \boldsymbol{\tau} \cdot \boldsymbol{\alpha})
$$

Do penúltimo termo de (1.7) temos uma relação de transformação

$$
\boldsymbol{\tau} \cdot \boldsymbol{\pi} \rightarrow \boldsymbol{\tau} \cdot \boldsymbol{\pi}^{\prime}=U \boldsymbol{\tau} \cdot \boldsymbol{\pi} U^{\dagger}
$$

$$
\tau^{j} \pi^{\prime j}=U \tau^{j} \pi^{j} U^{\dagger}
$$

com a finalidade de achar a forma em que $\tau^{i}$ se transforma sob $S U(2)$ vamos usar as 
propriedades das matrizes de Pauli, multiplicando por $\tau^{i}$

$$
\tau^{i} \tau^{j} \pi^{\prime j}=\tau^{i} U \tau^{j} \pi^{j} U^{\dagger}
$$

de onde

$$
\begin{gathered}
\operatorname{tr}\left(\tau^{i} \tau^{j} \pi^{\prime j}\right)=\operatorname{tr}\left(\tau^{i} U \tau^{j} \pi^{j} U^{\dagger}\right) \\
2 \pi^{i}=\operatorname{tr}\left(\tau^{i} U \tau^{j} U^{\dagger}\right) \pi^{j}, \\
\pi^{i}=\boldsymbol{R}^{i, j}(\boldsymbol{\alpha}) \pi^{j}, \quad \boldsymbol{R}^{i, j}(\boldsymbol{\alpha})=\frac{1}{2} \operatorname{tr}\left(\tau^{i} U \tau^{j} \pi^{j} U^{\dagger}\right),
\end{gathered}
$$

considerando $\boldsymbol{\alpha}$ infinitesimal mas dependendo do espaço-tempo

$$
\psi^{\prime}=(1-i \boldsymbol{\tau} \cdot \boldsymbol{\alpha}(x) / 2) \psi, \quad \pi^{i}=\pi^{i}-\epsilon^{i j k}\left(\partial_{\mu} \pi^{i}\right) \alpha^{k}(x),
$$

A seguir, achamos $\mathcal{L}\left(\psi^{\prime}, \boldsymbol{\pi}^{\prime}\right)$, para isto substituímos (1.12) em (1.7) e obtemos

$$
\mathcal{L}\left(\psi^{\prime}, \boldsymbol{\pi}^{\prime}\right)=\mathcal{L}(\psi, \boldsymbol{\pi})+\frac{1}{2} \bar{\psi} \gamma^{\mu} \boldsymbol{\tau} \cdot\left(\partial_{\mu} \boldsymbol{\alpha}\right) \psi-\epsilon^{i, j, k}\left(\partial_{\mu} \pi^{i}\right) \pi^{j} \partial^{\mu} \alpha^{k}
$$

A menos de termos de superfície, os termos remanescentes são

$$
\left.-\frac{1}{2} \partial^{\mu}\left(\bar{\psi} \gamma_{\mu} \tau^{k} \psi\right) \alpha^{k}+\partial^{\mu}\left[\epsilon^{i j k}\left(\partial_{\mu} \pi^{i}\right) \pi^{j}\right)\right] \alpha^{k}
$$

de onde identificamos o tripleto de correntes,

$$
V_{\mu}^{i}=\frac{1}{2} \bar{\psi} \gamma_{\mu} \tau^{k} \psi-\epsilon^{i j k}\left(\partial_{\mu} \pi^{i}\right) \pi^{j},
$$




\section{Simetria de Escala}

Uma transformação de escala dilata o espaço-tempo segundo

$$
\alpha: x \rightarrow x^{\prime}=e^{\alpha} x
$$

Elas agem linearmente sob os campos ${ }^{3}$ :

$$
\alpha: f(x) \rightarrow e^{\alpha d} f\left(e^{\alpha} x\right)
$$

onde d é alguma matriz [2] a transformação infinitesimal é

$$
\delta f=\left(d+x^{\mu} \partial_{\mu}\right) f
$$

Para o caso de bósons d será uma matriz que multiplica estes por 1 e no caso de férmions será de $\frac{3}{2}$. As transformações de escala são simetrias exatas no caso em que os campos não tem o termo de massa. Para ver isto, consideremos a lagrangiana do campo escalar real

$$
\mathcal{L}=\frac{1}{2} \partial_{\mu} \varphi \partial^{\mu} \varphi-\frac{1}{2} m^{2} \varphi^{2}
$$

Os termos desta lagrangiana vão se transformar segundo

$$
\begin{gathered}
\frac{1}{2} \partial_{\mu} \varphi \partial^{\mu} \varphi \rightarrow e^{4 \alpha} \partial_{\mu}^{\prime} \varphi\left(x^{\prime}\right) \partial^{\prime \mu} \varphi\left(x^{\prime}\right), \\
-\frac{1}{2} m^{2} \varphi^{2} \rightarrow-\frac{1}{2} m^{2} e^{2 \alpha} \varphi^{2}\left(x^{\prime}\right) .
\end{gathered}
$$

Só o termo de massa (1.21) faz com que a ação definida em (1.19) não seja invariante. Então só a simetria de escala é uma simetria exata do termo cinético nesta lagrangiana.

\footnotetext{
${ }^{3}$ aqui f representa um campo escalar o fermiônico
} 
A lagrangiana pode ser escrita em duas partes:

$$
\mathcal{L}=\mathcal{L}_{S}+\mathcal{L}_{B},
$$

onde $\mathcal{L}_{S}$ deixa a ação invariante de escala, mas $\mathcal{L}_{B}$ quebra a simetria de escala. A seguir vamos calcular as variações de $\mathcal{L}_{S}$ e $\mathcal{L}_{B}$,

$$
\begin{gathered}
\delta \mathcal{L}_{S}=\partial_{\mu}\left(x^{\mu} \mathcal{L}_{S}\right), \\
\delta \mathcal{L}_{B}=m^{2} \varphi^{2},
\end{gathered}
$$

de onde definimos a corrente de escala $s^{\mu}$, tão que:

$$
\partial_{\mu} s^{\mu}=m^{2} \varphi^{2} .
$$

É possível definir o tensor energia momento[2], $\theta^{\mu \nu}$ tão que

$$
s^{\mu}=x_{\nu} \theta^{\mu \nu} .
$$

Então a simetria de escala não é uma simetria exata ${ }^{4}$ em relação à lagrangiana definida em (1.19) e a corrente não é conservada, pois

$$
\theta_{\mu}^{\mu}=m^{2} \varphi^{2}
$$

O traço do tensor energia-momento representa uma quebra explícita da simetria de escala.

\footnotetext{
${ }^{4}$ Se a simetria de escala é fosse uma simetria exata então $\theta_{\mu}^{\mu}=0$
} 


\subsubsection{Simetria de Gauge}

É importante nos falar brevemente das simetrias de gauge, mais conhecidas como simetrias locais. A motivação é que na física do Modelo Padrão tais simetrias geram a dinâmica das interações onde se mistura o caso abeliano com o caso não abeliano.

\section{Simetria de Gauge caso Abeliano}

Seja $\psi(x)$ um campo fermiônico, considerar as transformações

$$
\begin{gathered}
\psi \rightarrow \psi^{\prime}=U(\alpha) \psi, \\
D_{\mu} \psi \rightarrow D_{\mu}^{\prime} \psi^{\prime}=U(\alpha) D_{\mu} \psi,
\end{gathered}
$$

onde $U(\alpha)$ forma um grupo abeliano com parâmetro de transformação $\alpha=\alpha(x)$. Podemos escrever a derivada convariante introduzindo um campo de gauge $A_{\mu}(x)$ segundo

$$
D_{\mu} \psi=\left(\partial_{\mu}+i f A_{\mu}\right) \psi,
$$

onde $f$ é a constante de acoplamento. Por exemplo na Eletrodinâmica Quântica (QED), neste caso $f$ é a carga eletrônica. Agora só precisamos saber como o campo $A_{\mu}(x)$ vai se transformar sob a transformação de gauge. Para isso usamos (1.27),

$$
\begin{gathered}
D_{\mu}^{\prime} \psi^{\prime}=U(\alpha)\left(\partial_{\mu}+i f A_{\mu}\right) U^{-1}(\alpha) \psi^{\prime}, \\
D_{\mu}^{\prime} \psi^{\prime}=U(\alpha)\left(U^{-1} \partial_{\mu}+\partial_{\mu} U^{-1}(\alpha)+i f A_{\mu} U^{-1}(\alpha)\right) \psi^{\prime},
\end{gathered}
$$

tendo em conta a forma da derivada covariante (1.28), temos que

$$
\left(\partial_{\mu}+i f A_{\mu}^{\prime}\right) \psi^{\prime}=U(\alpha)\left(U^{-1} \partial_{\mu}+\partial_{\mu} U^{-1}(\alpha)+i f A_{\mu} U^{-1}(\alpha)\right) \psi^{\prime},
$$


de onde concluímos que

$$
A_{\mu}^{\prime}=A_{\mu}+\frac{i}{f}\left(\partial_{\mu} U(\alpha)\right) U^{-1}(\alpha) .
$$

O campo $A_{\mu}$ tem que se propagar. Para isso definimos o comutador das derivadas covariantes,

$$
\left[D_{\mu}, D_{\nu}\right] \psi=i f F_{\mu \nu} \psi,
$$

onde

$$
F_{\mu \nu}=\partial_{\mu} A_{\nu}-\partial_{\nu} A_{\mu},
$$

Notar de (1.27) e (1.29) que $F_{\mu \nu}$ é invariante sob as transformações de gauge, e então a lagrangiana de um férmion invariante sob transformações de gauge $U(1)$ tem a forma:

$$
\mathcal{L}=-\frac{1}{4} F_{\mu \nu} F^{\mu \nu}+i \bar{\psi} \not D \psi-m \bar{\psi} \psi
$$

\section{Simetria de Gauge caso não Abeliano}

São transformações que envolvem graus de liberdade internos. Foram Yang e Mills (1954) que estenderam o princípio de gauge a simetrias não abelianas, e por isso são conhecidas como teorias de Yang Mills. Elas tem uma complicação adicional em relação ao caso abeliano. Considerando os campos

$$
\Psi=\left(\begin{array}{c}
\Psi_{1} \\
\Psi_{2} \\
\cdot \\
\cdot \\
\cdot \\
\Psi_{\mathrm{r}}
\end{array}\right),
$$


matrizes $\mathrm{r} \times \mathrm{r}$ operam sob $\Psi$. Consideremos também um grupo de Lie com n geradores $t_{a}$, tais que obedecem a álgebra

$$
\left[t^{a}, t^{b}\right]=i c^{a b c} t^{c} \quad(a, b, c=1, \ldots, n)
$$

$\operatorname{com} c^{a b c}$ sendo as constantes de estrutura da álgebra. Obtemos a seguinte lei de transformação

$$
\Psi^{\prime}=U(\vec{\alpha}) \Psi
$$

com

$$
U(\vec{\alpha})=\exp \left(-i \alpha^{a}(x) t^{a}\right)
$$

e onde a derivada covariante é expressada em termos dos campos de gauge $A_{\mu}^{a}$, segundo

$$
D_{\mu} \Psi=\left(I \partial_{\mu}+i g A_{\mu}\right) \psi
$$

Aqui g é a constante de acoplamento, $I$ é a matriz identidade $\mathrm{r} \times \mathrm{r}$ e $A_{\mu}=t^{a} A_{\mu}^{a}$, a transformação da derivada covariante é

$$
\left(D_{\mu} \Psi\right)^{\prime}=U(\vec{\alpha}) D_{\mu} \Psi
$$

usando (1.38), (1.37) e (1.36), obtemos na forma de matriz

$$
A_{\mu}^{\prime}=U(\vec{\alpha}) A_{\mu} U^{-1}(\vec{\alpha})+\frac{i}{g}\left(\partial_{\mu} U(\vec{\alpha})\right) \cdot U^{-1}(\vec{\alpha})
$$

Para achar o campo $F_{\mu \nu}$ primeiro achamos o comutador das derivadas covariantes,

$$
\left[D_{\mu}, D_{\nu}\right] \Psi=i g F_{\mu \nu} \Psi
$$


de onde

$$
F_{\mu \nu}=\partial_{\mu} A_{\nu}-\partial_{\nu} A_{\mu}+i g\left[A_{\mu}, A_{\nu}\right]
$$

$F_{\mu \nu}$ não vai ser invariante sob a transformação de gauge não abeliano, isto é usando (1.41), (1.39) obtemos

$$
F_{\mu \nu}^{\prime}=U(\vec{\alpha}) F_{\mu \nu} U^{-1}(\vec{\alpha})
$$

Então a lagrangiana invariante de gauge que tem os termos cinéticos nos campos $A_{\mu}$ é

$$
\mathcal{L}=-\frac{1}{4} \operatorname{Tr}\left(F_{\mu \nu} F^{\mu \nu}\right)+i \bar{\Psi} \not D \Psi-m \bar{\Psi} \Psi
$$

\subsection{Cromodinâmica Quântica (QCD)}

O setor das interações fortes do Modelo Padrão mais conhecido como QCD, é uma teoria de gauge não abeliana onde os geradores pertencem ao grupo de simetria local $S U(3)_{C}$, e o grau de liberdade interna é a chamada de cor. Uma das propriedades da QCD é a liberdade assintótica que faz possível usar métodos perturbativos a distâncias muito pequenas [1]. A lagrangiana da QCD é

$$
\mathcal{L}=-\frac{1}{4} F_{\mu \nu}^{i} F^{\mu \nu i}+\sum_{r} \bar{q}_{r \alpha} i \not D_{\mu \beta}^{\alpha} q_{r}^{\beta},
$$

onde $r$ é o índice de soma sob os sabores dos quarks; o índice $i=1,2, \ldots, 8$ dos 8 geradores de $S U(3)_{C} ; \quad \alpha, \beta=1,2,3$ são os índices de cor, e $F_{\mu \nu}^{i}$ é o tensor intensidade do campo de gauge que é dado por

$$
F_{\mu \nu}^{i}=\partial_{\mu} G_{\nu}^{i}-\partial_{\nu} G_{\mu}^{i}-g_{s} f_{i j k} G_{\mu}^{j} G_{\nu}^{k}
$$

onde os campos $G_{\mu}^{i}$ são os campos dos glúons e as constantes de estrutura $f_{i j k} \quad(i, j, k=$ 
$1,2, \ldots, 8)$ são definidas por

$$
\left[\lambda^{i}, \lambda^{j}\right]=2 i f_{i j k} \lambda^{k}
$$

As matrizes $\lambda^{k}$ são as matrizes de Gell-Mann para $S U(3)$. A derivada covariante é dada por

$$
D_{\mu \beta}^{\alpha}=\partial_{\mu} \delta_{\beta}^{\alpha}+\frac{1}{2} i g_{s} G_{\mu}^{i} \lambda_{\beta}^{\alpha i},
$$

sendo $g_{s}$ o parâmetro de acoplamento de gauge $S U(3)$.

\subsection{Modelo eletrofraco}

O modelo de Glashow-Weinberg-Salam(GWS) onde os férmions são quirais é uma teoria de gauge com simetria $S U(2)_{L} \times U(1)_{Y}$, onde $L$ e $Y$ representam respectivamente a quiralidade de mão esquerda com simetria de isospin fraco, e hipercarga fraca.

Aqui os léptons de mão esquerda são

$$
\mathrm{L}_{e}=\left(\begin{array}{c}
\nu_{e} \\
e^{-}
\end{array}\right)_{L} \quad \mathrm{~L}_{\mu}=\left(\begin{array}{c}
\nu_{\mu} \\
\mu^{-}
\end{array}\right)_{L} \quad \mathrm{~L}_{\tau}=\left(\begin{array}{c}
\nu_{\tau} \\
\tau^{-}
\end{array}\right)_{L},
$$

com isospin fraco $I_{\ell}=1 / 2$ e hipercarga $Y_{L_{\ell}}=-1$, e pelos léptons de mão-direita

$$
\mathrm{R}_{e, \mu, \tau}=e_{R}, \mu_{R}, \tau_{R}
$$

com hipercargas $Y_{R_{\ell}}=-2$ são fixadas pela relação de Gell-Mann e Nishijima $Q=$ $I_{3}+(1 / 2) Y$. Onde assumimos que não temos neutrinos de mão direita.

O setor de quarks consiste dos quarks de mão-esquerda

$$
\mathrm{L}_{q}^{1}=\left(\begin{array}{c}
u \\
d
\end{array}\right)_{L} \quad \mathrm{~L}_{q}^{2}=\left(\begin{array}{c}
c \\
s
\end{array}\right)_{L} \quad \mathrm{~L}_{q}^{3}=\left(\begin{array}{c}
t \\
b
\end{array}\right)_{L},
$$

com isospin fraco $I_{q}=1 / 2$ e hipercarga $Y_{L_{q}}=1 / 3$, e dos quarks de mão-direita 


$$
\begin{aligned}
& \mathrm{R}_{u}^{(1,2,3)}=u_{R}, c_{R}, t_{R}, \\
& \mathrm{R}_{d}^{(1,2,3)}=d_{R}, s_{R}, b_{R},
\end{aligned}
$$

com hipercargas $Y_{R_{u}}=4 / 3$ e $Y_{R_{d}}=-2 / 3$.

Escrevemos a lagrangiana que é invariante de gauge $S U(2)_{L} \times U(1)_{Y}$, que tem três partes, de gauge $(G)$, férmions $(F)$, e do Higgs $(H)$,

$$
\mathcal{L}=\mathcal{L}_{G}+\mathcal{L}_{F}+\mathcal{L}_{H}
$$

com

$$
\mathcal{L}_{G}=-\frac{1}{4} W_{\mu \nu}^{i} W^{\mu \nu i}-\frac{1}{4} B_{\mu \nu} B^{\mu \nu},
$$

onde o tensor $W_{\mu \nu}^{i}$ corresponde a $S U(2)$ com acoplamento $g$ é

$$
W_{\mu \nu}^{i}=\partial_{\nu} W_{\mu}^{i}-\partial_{\mu} W_{\nu}^{i}+g \varepsilon_{i j k} W_{\mu}^{j} W_{\nu}^{k}
$$

e o tensor $B_{\mu \nu}$ correspondente a U(1), com acoplamento $g^{\prime}$ é

$$
B_{\mu \nu}=\partial_{\nu} B_{\mu}-\partial_{\mu} B_{\nu}
$$

As lagrangianas dos férmions é escrita em duas partes

$$
\mathcal{L}=\mathcal{L}_{\text {léptons }}+\mathcal{L}_{\text {quarks }},
$$

$$
\mathcal{L}_{\text {léptons }}=\overline{\mathrm{R}}_{\ell} i \gamma^{\mu}\left(\partial_{\mu}+i \frac{g^{\prime}}{2} B_{\mu} Y\right) \mathrm{R}_{\ell}+\overline{\mathrm{L}}_{\ell} i \gamma^{\mu}\left(\partial_{\mu}+i \frac{g^{\prime}}{2} B_{\mu} Y+i \frac{g}{2} \vec{\sigma} \cdot \vec{W}_{\mu}\right) \mathrm{L}_{\ell},
$$




$$
\begin{aligned}
\mathcal{L}_{\text {quarks }}=\overline{\mathrm{R}}_{u}^{n} i \gamma^{\mu}\left(\partial_{\mu}+i \frac{g^{\prime}}{2} B_{\mu} Y\right) \mathrm{R}_{u}^{n} & +\overline{\mathrm{R}}_{d}^{n} i \gamma^{\mu}\left(\partial_{\mu}+i \frac{g^{\prime}}{2} B_{\mu} Y\right) \mathrm{R}_{d}^{n} \\
& +\overline{\mathrm{L}}_{q}^{n} i \gamma^{\mu}\left(\partial_{\mu}+i \frac{g^{\prime}}{2} B_{\mu} Y+i \frac{g}{2} \vec{\sigma} \cdot \vec{W}_{\mu}\right) \mathrm{L}_{q}^{n},
\end{aligned}
$$

sendo que o índice $\ell$ representa os léptons $(\ell=e, \mu, \tau)$, o índice $n$ representa as gerações $(n=1,2,3)$ e $\vec{\sigma}$ representa as matrizes de Pauli: $\sigma_{1}=\left(\begin{array}{cc}0 & 1 \\ 1 & 0\end{array}\right), \sigma_{2}=\left(\begin{array}{cc}0 & -i \\ i & 0\end{array}\right)$ e $\sigma_{3}=\left(\begin{array}{cc}1 & 0 \\ 0 & -1\end{array}\right)$

A lagrangiana (1.54) tem quatro bósons de gauge sem massa $W_{\mu}^{1}, W_{\mu}^{2}, W_{\mu}^{3}$ e $B_{\mu}$. Eles são não massivos porque seus termos de massa não são invariantes sobre transformações de gauge. Além disso, a simetria de gauge $S U(2)_{L} \times U(1)_{Y}$ proíbe termos de massa para os férmions já que as componentes de mão-esquerda e de mão-direita dos campos fermiônicos se transformam de forma diferentes.

Mas a natureza mostra que os férmions tem massa, é por isso que os bósons de gauge e os férmions adquirem massa via o mecanismo de Higgs. Isso é feito pela introdução de um dubleto de campos escalares complexos

$$
\Phi=\left(\begin{array}{c}
\phi^{+} \\
\phi^{0}
\end{array}\right)
$$

e hipercarga $Y_{\Phi}=1$. Assim se $H$ é o campo do Higgs, ele tem que acoplar-se com os campos de gauge e os férmions, e $\mathcal{L}_{H}$ pode ser escrita como

$$
\mathcal{L}_{H}=\mathcal{L}_{H G}+\mathcal{L}_{H F},
$$

onde

$$
\mathcal{L}_{H G}=\left(D^{\mu} \Phi\right)^{\dagger}\left(D_{\mu} \Phi\right)-V\left(\Phi^{\dagger} \Phi\right),
$$


e

$$
D_{\mu}=\partial_{\mu}+i \frac{g^{\prime}}{2} B_{\mu} Y+i \frac{g}{2} \vec{\sigma} \cdot \vec{W}_{\mu}
$$

O potencial de auto-interação do dubleto escalar é dado por

$$
V\left(\Phi^{\dagger} \Phi\right)=\mu^{2}\left(\Phi^{\dagger} \Phi\right)+\lambda\left(\Phi^{\dagger} \Phi\right)^{2}
$$

Também temos de (1.61) que as interações de Yukawa entre o Higgs com os léptons carregados e os quarks pode ser escrita como

$$
\begin{gathered}
\mathcal{L}_{H F}=\mathcal{L}_{\mathrm{Y}(\text { léptons })}+\mathcal{L}_{\mathrm{Y}(\text { quarks })}, \\
\mathcal{L}_{\mathrm{Y}(\text { léptons })}=-G_{\ell}\left[\left(\overline{\mathrm{L}}_{\ell} \Phi\right) \mathrm{R}_{\ell}+\overline{\mathrm{R}}_{\ell}\left(\Phi^{\dagger} L_{\ell}\right)\right] \\
\mathcal{L}_{\mathrm{Y} \text { (quarks) }}=-\sum_{i, j=1}^{3}\left[G_{i j}^{u} \overline{\mathrm{R}}_{u}^{i}\left(\tilde{\Phi}^{\dagger} \mathrm{L}_{q}^{j}\right)+G_{i j}^{d} \overline{\mathrm{R}}_{d}^{i}\left(\Phi^{\dagger} \mathrm{L}_{q}^{j}\right)+\text { h.c. }\right],
\end{gathered}
$$

onde $G_{\ell}, G_{i j}^{u}$ e $G_{i j}^{d}$ são acoplamentos de Yukawa e $\tilde{\Phi} \equiv i \sigma_{2} \Phi^{*}$. Notamos que todos esses termos também são invariantes de gauge.

A seguir vamos trabalhar com o estado fundamental que minimiza o potencial (1.64).

Para $\mu^{2}<0$ (caso não trivial) a simetria eletrofraca é espontaneamente quebrada. Se tem como solução não trivial para $\Phi$,

$$
\langle\Phi\rangle_{0}=\left(\begin{array}{c}
0 \\
v / \sqrt{2}
\end{array}\right),
$$

onde $v=\sqrt{-\mu^{2} / \lambda}$. Usando o Modelo $\sigma$ não Linear[3], parametrizamos o campo $\Phi$,

$$
\Phi=e^{i \frac{\xi^{j}(x) \sigma_{j}}{2 v}}\left(\begin{array}{c}
0 \\
\frac{v+h(x)}{\sqrt{2}}
\end{array}\right),
$$

onde $\sigma_{j}$ são as matrizes de Pauli. Mas vamos trabalhar no gauge unitário tal que 


$$
\Phi \rightarrow e^{-i \frac{\xi^{j}(x) \sigma_{j}}{2 v}} \Phi=\left(\begin{array}{c}
0 \\
\frac{v+h(x)}{\sqrt{2}}
\end{array}\right),
$$

então a $\mathcal{L}_{H G}(1.62)$ pode ser escrita em termos dos campos físicos como

$$
\begin{array}{r}
\mathcal{L}_{H G}=\frac{1}{2} \partial_{\mu} h \partial^{\mu} h+\frac{g^{2}}{4}(v+h)^{2}[ \\
{\left[W_{\mu}^{+} W^{-\mu}+\frac{1}{2 \cos ^{2} \theta_{W}} Z_{\mu} Z^{\mu}\right]} \\
-\mu^{2} \frac{(v+h)^{2}}{2}-\lambda \frac{(v+h)^{4}}{4} .
\end{array}
$$

Temos definido os campos mediadores das interações fracas carregadas $W_{\mu}^{ \pm}$,

$$
W_{\mu}^{ \pm}=\left(W_{\mu}^{1} \mp i W_{\mu}^{2}\right) / \sqrt{2} .
$$

esses campos físicos $W_{\mu}^{ \pm}$adquirem massas iguais a $M_{W}=g v / 2=e v / 2 \sin \theta_{W}$ e o campo que media as interações fracas neutras $Z_{\mu}$,

$$
Z_{\mu}=W_{\mu}^{3} \cos \theta_{W}-B_{\mu} \sin \theta_{W}
$$

adquire uma massa de $M_{Z}=M_{W} / \cos \theta_{W} ; \theta_{W}$ é o ângulo de Weinberg definido pela relação $\tan \theta_{W} \equiv g^{\prime} / g$. O campo do fóton, escrito como a combinação $A_{\mu}=B_{\mu} \cos \theta_{W}+$ $W_{\mu}^{3} \sin \theta_{W}$, não adquire massa.

Portanto, o valor esperado de vácuo (1.68) quebra a simetria de gauge $S U(2)_{L} \times U(1)_{Y}$ $\longrightarrow U(1)_{\mathrm{em}}$.

A teoria eletrofraca faz a previsão que a baixas energias, obtemos a teoria de fermi (V-A). Isto é ao comparar ambas lagrangianas dessas teorias nesse limite é possível obter que $v=\left(\sqrt{2} G_{F}\right)^{-1 / 2} \simeq 246 \mathrm{GeV}$, onde $G_{F}$ é constante de acoplamento de Fermi da interação fraca. Portanto, o Modelo Padrão dá previsões para as massas dos bósons $W^{ \pm}$e $Z$

$$
M_{W}^{2}=\frac{e^{2} v^{2}}{4 \sin ^{2} \theta_{W}} \simeq(80 \mathrm{GeV})^{2}
$$




$$
M_{Z}^{2}=\frac{M_{W}^{2}}{\cos ^{2} \theta_{W}} \simeq(90 \mathrm{GeV})^{2}
$$

tendo em conta o valor experimental de $\sin ^{2} \theta_{W} \simeq 0.22$.

A seguir vamos considerar a lagrangiana $\mathcal{L}_{H F}(1.65)$ e logo de substituir $\Phi$ (1.70) em (1.66) temos que

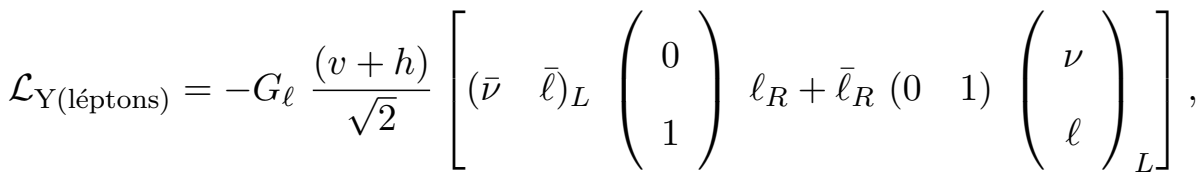

$$
\begin{aligned}
& \mathcal{L}_{\mathrm{Y}(\text { léptons })}=-\frac{G_{\ell} v}{\sqrt{2}} \bar{\ell} \ell-\frac{G_{\ell}}{\sqrt{2}} \bar{\ell} \ell h,
\end{aligned}
$$

onde $\ell=e, \mu, \tau$. Identificamos de (1.74) que a massa dos léptons carregados é dada por $M_{\ell}=G_{\ell} v / \sqrt{2}$. Com esse procedimento é possível obter os termos de massa para os léptons de maneira invariante de gauge. Entretanto, o valor da massa $M_{\ell}$ não é previsto pela teoria já que os acoplamentos de Yukawa $\left(G_{\ell}\right)$ foram introduzidos arbitrariamente para reproduzir o espectro de massas dos léptons observados.

Agora vamos a considerar a lagrangiana (1.59), isto é logo de substituir $\Phi$ (1.70) em (1.67)

$$
\bar{u}_{R}^{i} M_{i j}^{U} u_{L}^{j}+\text { h.c. }
$$

onde $\bar{u}_{R}^{i}=\left\{\bar{u}_{R}, \bar{c}_{R}, \bar{t}_{R}\right\}$ e $u_{L}^{j}$ é a componente up do dubleto de quarks $\mathrm{L}_{q}^{j}(1.50)$.

Para os quarks down temos também

$$
\bar{d}_{R}^{i} M_{i j}^{D} d_{L}^{j}+\text { h.c., }
$$

onde $\bar{d}_{R}^{i}=\left\{\bar{d}_{R}, \bar{s}_{R}, \bar{b}_{R}\right\}$ e $d_{L}^{j}$ é a componente down do dubleto de quarks $\mathrm{L}_{q}^{j}(1.50)$. As matrizes $M^{U}$ e $M^{D}$ são matrizes não diagonais dadas por $M_{i j}^{U(D)}=G_{i j}^{U(D)} v / \sqrt{2}$. Como para o caso dos léptons, a teoria não prevê os valores das massas $M_{i j}^{U(D)}$ já que 
os acoplamentos de Yukawa $G_{i j}^{U(D)}$ foram introduzidos de maneira arbitrária para dar corretamente o espectro de massas dos quarks observados.

Vamos definir as matrizes $U(D)_{L, R}$ unitárias tais que:

$$
\begin{array}{ll}
u_{L}^{i}=U_{L}^{i j} u_{L}^{\prime j}, & d_{L}^{i}=D_{L}^{i j} d_{L}^{\prime j}, \\
u_{R}^{i}=U_{R}^{i j} u_{R}^{\prime j}, & d_{R}^{i}=D_{R}^{i j} d_{R}^{\prime j},
\end{array}
$$

Notar que os auto-estados $(q)$ são combinações lineares dos auto-estados de massa ${ }^{5}$ $\left(q^{\prime}\right)$, então temos que fazer uma mudança de base a fim de diagonalizar $M^{U}$ e $M^{D}$,

$$
\begin{gathered}
M_{\text {diag }}^{U} \equiv U_{R}^{\dagger} M^{U} U_{L}=\left(\begin{array}{ccc}
m_{u} & 0 & 0 \\
0 & m_{c} & 0 \\
0 & 0 & m_{t}
\end{array}\right), \\
M_{\text {diag }}^{D} \equiv D_{R}^{\dagger} M^{D} D_{L}=\left(\begin{array}{ccc}
m_{d} & 0 & 0 \\
0 & m_{s} & 0 \\
0 & 0 & m_{b}
\end{array}\right) .
\end{gathered}
$$

Para o caso das correntes carregadas temos que devem ser proporcionais a

$$
\left(\begin{array}{ccc}
\bar{u}^{\prime} & \bar{c}^{\prime} & \bar{t}^{\prime}
\end{array}\right)_{L} U_{L}^{\dagger} \gamma_{\mu} D_{L}\left(\begin{array}{c}
d^{\prime} \\
s^{\prime} \\
b^{\prime}
\end{array}\right)_{L}
$$

ou seja, o acoplamento da corrente carregada não será mais diagonal, já que $U_{L}^{\dagger} D_{L} \neq \mathbf{1}_{3}$. Essa matriz unitária que expressa a mistura entre os quarks é conhecida como matriz de Cabibbo-Kobayashi-Maskawa

$$
\mathrm{V}_{\mathrm{CKM}} \equiv U_{L}^{\dagger} D_{L}=\left(\begin{array}{ccc}
V_{u d} & V_{u s} & V_{u b} \\
V_{c d} & V_{c s} & V_{c b} \\
V_{t d} & V_{t s} & V_{t b}
\end{array}\right)
$$

No caso das correntes neutras, devem ser proporcionais a

\footnotetext{
${ }^{5}$ Para confrontar o experimento com a teoria se usa os auto-estados de massa.
} 


$$
\left(\begin{array}{ccc}
\bar{u}^{\prime} & \bar{c}^{\prime} & \bar{t}^{\prime}
\end{array}\right)_{L} U_{L}^{\dagger} \gamma_{\mu} U_{L}\left(\begin{array}{c}
u^{\prime} \\
c^{\prime} \\
t^{\prime}
\end{array}\right)_{L}
$$

Então não há mistura de quarks no setor de correntes neutras, devido a que $U_{L}$ é unitária ou seja, os acoplamentos são diagonais. Portanto, no MP não existem interações que mudam sabor a nível árvore.

\subsection{Motivação da Física Além do Modelo Padrão}

Nas seções anteriores vimos que o Modelo Padrão das partículas elementares proporciona uma descrição teórica das interações fortes, fracas e eletromagnéticas. Ela é extremamente bem sucedida quando é comparada com os dados experimentais com que nos contamos hoje, isto é a detecção das correntes neutras na década dos 70 , as previsões feitas para as massas dos bósons $W_{\mu}^{ \pm}$e $Z_{\mu}$, experimentos feitos no LePI, LePII e o Tevatron [4], [5] com grande precisão $(\lesssim 1 \%)$ como é para o caso do parâmetro $\varrho_{0}$

$$
\varrho_{0}=\frac{m_{W}^{2}}{m_{Z}^{2} \cos \theta_{W}},
$$

sendo $\varrho_{0}=1$ a nível de árvore, o MP prevê $\varrho_{0}$ até 2 loop. O modelo precisa de uma partícula escalar remanescente do processo da quebra espontânea da simetria do setor eletrofraco, o bóson de Higgs que se acopla com as outras partículas do modelo, e que ainda não foi descoberta ${ }^{6}$.

Além disso o modelo tem deficiências em como explicar o problema da hierarquia de gauge e o problema da hierarquia da massa dos férmions, relacionados com a instabili-

\footnotetext{
${ }^{6}$ As procuras da massa do bóson de Higgs fazem com que $M_{h}>114,4 G e V$ [6] excluindo entre $158 \mathrm{GeV}$ e $175 \mathrm{GeV}[7]$ com $95 \%$ de nível de confiança.
} 
dade quântica do vácuo do Higgs que fixa a escala eletrofraca ao redor de $m_{E W} \sim 1 T e V$. Notar também que o Modelo Padrão não inclui a interação com a gravidade.

\subsubsection{Problema da Hierarquia de Gauge}

Desde que o Modelo Padrão não é uma teoria definitiva por não ter incluído a gravidade, vamos considerar a escala conhecida como escala de Planck

$$
M_{\text {Planck }}=\left(\frac{\hbar c}{G_{\text {Newton }}}\right)^{1 / 2} \simeq 1,2 \times 10^{19} \mathrm{GeV}
$$

onde os efeitos gravitacionais quânticos serão importantes. A pergunta é: até que escala de energia o modelo tem validade? Sabemos que o Modelo Padrão precisa do bóson de Higgs, e que esta partícula tem que aparecer na escala Tev por unitaridade [1]. Assim a escala da quebra da simetria eletrofraca é dada pelo VEV do $\operatorname{Higgs}(v \simeq 246 G e V)$.

O problema é que a massa do Higgs não é naturalmente estável sob correções radiativas. Para entender isto vamos considerar as correções a um loop para a massa do Higgs proveniente dos loops que contêm os bósons de gauge $\left(W_{-}^{+}\right.$e $\left.Z\right)$, auto-acoplamento do Higgs e férmions (a contribuição dominante é a do quark top) cujos diagramas correspondentes estão na Figura(1.1). As correções [8] resultam em

$$
\delta M_{h}^{2} \simeq \Lambda^{2} \frac{3\left(2 M_{W}^{2}+M_{Z}^{2}+M_{h}^{2}-4 M_{t}^{2}\right)}{32 \pi^{2} v^{2}}
$$

mais termos que dependem do logaritmo da escala $\Lambda$. Então a correção à massa do Higgs é quadraticamente sensível ao corte da teoria, e se o Modelo Padrão tivesse validade até a escala de Planck, a massa do Higgs alcançaria a escala de Planck por correções radiativas. Vamos precisar um ajuste fino para lograr que a massa física do Higgs esteja na escala eletrofraca, e esse ajuste não é natural e precisa de nova física além do Modelo Padrão para restabelecer naturalidade [9] na teoria. 
a)

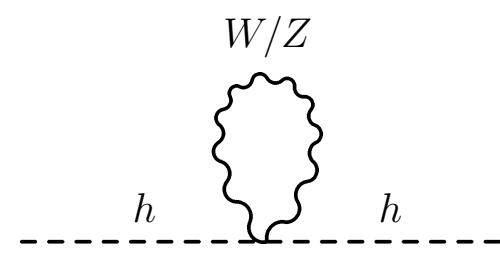

c)

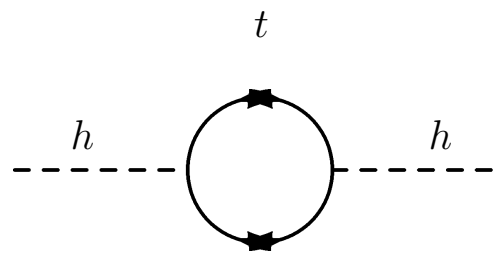

$t$ b)

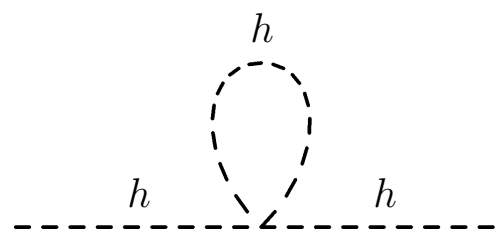

Figura 1.1: Correções radiativas à massa do Higgs: a) loops dos bósons de gauge; b) auto-acoplamento do Higgs; c) loop de férmions( quark top)

\subsubsection{Problema da Hierarquia da Massa dos férmions}

Quando revisamos a seção relacionada ao setor eletrofraco do Modelo Padrão, especificamente quando via o mecanismo de Higgs $\Phi$ adquiriu um valor esperado de vácuo, nós conseguimos termos de massa. Isto é nas interações de Yukawa (1.66) e (1.67), os termos de massa podem ser identificados como

$$
M_{f}=\frac{G_{f} v}{\sqrt{2}},
$$

onde $G_{f}$ são os acoplamentos de Yukawa dos férmions. Estes são extremamente variados. Por exemplo temos os acoplamentos para o elétron e o top respectivamente $G_{e} \sim 10^{-6}$ e $G_{t} \sim 1$, sendo este o ponto do problema já que o MP não explica porque 
os férmions podem ter massas tão diferentes sendo que as massas de essas partículas foram geradas através do mecanismo de Higgs. Portanto para explicar o fato de esses acoplamentos serem tão diferentes precisa de física além do Modelo Padrão.

\subsubsection{Outros Problemas}

Vamos mencionar de um jeito não rigoroso certos problemas que não são resolvidos pelo Modelo Padrão.

\section{Problema da Constante Cosmológica}

Este problema se refere ao fato que o valor da constante cosmológica [10] teórico é muito maior do que a observada experimentalmente. Isto é a densidade de energia do vácuo teórica relacionada com a constante cosmológica através de $\Lambda=8 \pi G \rho_{\text {vácuo }}$ é 120 ordens de grandeza maior do que observado experimentalmente.

\section{Problema da Matéria Escura}

O modelo Padrão não tem candidato a matéria escura, mas sabemos que este tipo de matéria é pesado, de carga neutras e estável e que apenas interage gravitacionalmente com a matéria bariônica.

\section{Problema da assimetria Matéria-Anti-matéria}

A assimetria observada no universo entre a matéria e a antimatéria não pode ser explicada pelo Modelo Padrão. Para quantificar esta assimetria se usa a razão $\eta$

$$
\eta \equiv \frac{n_{b}-n_{\bar{b}}}{n_{\gamma}}=(6,22 \pm 0,19) \times 10^{-10}
$$

onde $n_{b}, n_{\bar{b}}$, e $n_{\gamma}$ são as densidades numéricas dos bárions, dos anti-bárions e dos fótons, respectivamente. 


\section{Problema de Violação de CP nas Interações Fortes}

A simetria $C P$ transforma um estado de uma partícula de mão esquerda, em um estado de sua anti-partícula de mão direita; então podemos pensar que a quebra dessa simetria nos ajudara a elucidar o problema da assimetria matéria-antimatéria. Na lagrangiana da QCD existe um termo que viola $C P$ dado por

$$
i \theta \frac{g^{2}}{32 \pi^{2}} G_{\mu \nu}^{a} \tilde{G}^{a \mu \nu},
$$

onde $\theta$ é o coeficiente determinado experimentalmente, sendo que $g$ é o acoplamento associado com $S U(3)$. Uma tentativa de solução foi proposta por Peccei e Quinn [11] por meio da introdução de uma nova partícula hipotética denominada áxion.

O problema da hierarquia ( e talvez o da hierarquia das massas dos férmions ) parece ser uma boa guia para construir teorias Além do MP.

Vamos considerar teorias que resolvem o Problema da hierarquia gerando grandes hierarquias de escalas de uma forma natural. 


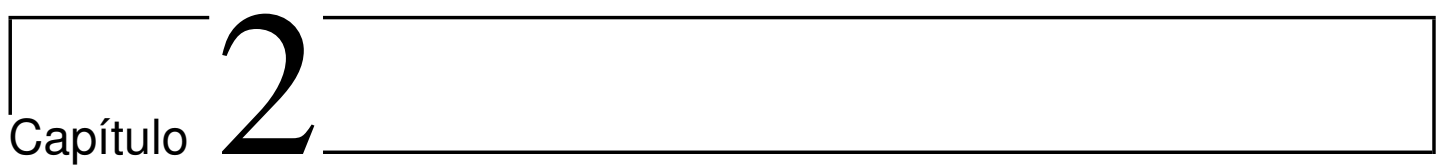

\section{Teorias Quiver a partir de Teorias com}

\section{uma Dimensão Extra}

A idéia de considerar dimensões espaciais extras, além das quatro dimensões usuais, na física teórica é motivada pelo uso sistemático das teorias de Kaluza [12] e Klein [13] no começo dos anos 1920. Eles trabalharam independentemente, com o objetivo de unificar a gravitação com o eletromagnetismo usando uma dimensão extra compacta. Mais recentemente, as dimensões extras são ingredientes fundamentais na teoria de cordas que tenta unificar todas as interações fundamentais conhecidas da matéria com a gravitação. Por outro lado, em física de partículas interessa recentemente usar dimensões extras curvas (warped extra dimensions) no cenário de Randall-Sundrum(RS) [14] no contexto de Física Além do Modelo Padrão, porque é possível gerar naturalmente a grande diferença entre as escalas de energias de Planck e fraca, assim como também explicar o problema da hierarquia das massas dos férmions, como apresentaremos no presente capítulo. Estudos de violação de sabor em teorias $\mathrm{AdS}_{5}$, são teorias com uma dimensão extra compacta com métrica $\mathrm{AdS}$ que vamos denotar como $\mathrm{AdS}_{5}$, tem mostrado que essas teorias violam sabor a nível árvore, entretanto a desconstrução dimensional resolve os problemas da hierarquia de gauge e da hierarquia da massas dos 
férmions com mínima violação de sabor [19].

Brevemente, na seguinte seção nós revisaremos as teorias $\mathrm{AdS}_{5}$ como um preâmbulo para construir teorias $4 D$ com grandes hierarquias de escala através do mecanismo de Desconstrução. Essas teorias 4D, chamadas de Teorias Quiver de Hierarquia Completa $(T Q H C)$ são capazes de resolver os problemas da hierarquia de gauge e das massas dos férmions de forma similar que as teorias $\mathrm{AdS}_{5}$, porém essas teorias são fundamentalmente diferentes, isto é, veremos que as teorias $\mathrm{AdS}_{5}$ podem ser obtidas a partir de teorias puramente $4 D$. 


\subsection{Teorias $\mathrm{AdS}_{5}$ com métrica de RS com cam- pos na Bulk 5D}

De acordo com o modelo proposto por Lisa Randall e Raman Sundrum [14], a dimensão extra $y$ é compactificada no orbifold $S_{1} / Z_{2}$. Isto significa que a dimensão extra tem a periodicidade do círculo $\left(S_{1}\right)$, equivalentemente que o mundo na dimensão extra vai se repetir cada longitude $2 \pi r_{c}$,

$$
y+2 \pi r_{c} \rightarrow y,
$$

onde $r_{c}$ é o radio da dimensão extra, fazendo $L=\pi r_{c}$ portanto $y$ está limitado ao intervalo $-L \leq y \leq L$, adicional-mente dentro do círculo a simetria discreta $Z_{2}$ implementa a transformação de paridade sob a dimensão extra

$$
y \rightarrow-y,
$$

o que faz possível a identificação $(x, y)$ com $(x,-y)$, assim só vamos considerar o intervalo $0 \leq y \leq L$. A compactificação é representada na Figura (2.1). Os pontos $y=0, L$ que são invariantes sob $Z_{2}$ serão considerados como localização das duas 3branas $^{1}$. A brana de Planck ou UV (ultravioleta) e a brana TeV ou IR(Infravermelha) são localizadas em $y=0$ e $y=L$ respectivamente.

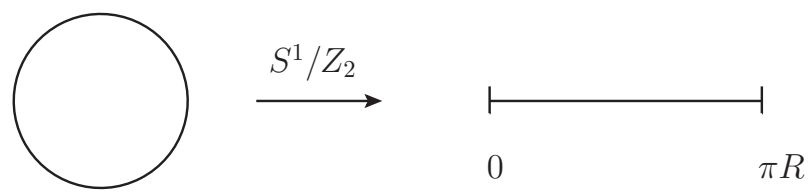

Figura 2.1: Compactificação em orbifold.

O modelo tem a métrica anti-de Sitter $\left(A d S_{5}\right)$ em cinco dimensões da forma

$$
d s^{2}=g_{M N} d x^{M} d x^{N}=e^{-2 k|y|} \eta_{\mu \nu} d x^{\mu} d x^{\nu}-d y^{2},
$$

\footnotetext{
${ }^{1}$ As 3-branas podem ter teorias de campos em (3+1)-dimensões [14].
} 
onde $k$ é a curvatura $\mathrm{AdS}_{5}$, as letras latinas e gregas são índices 5-dimensionais e 4dimensionais respectivamente, $(M, N=\mu, \nu, 5)$; e $\eta_{\mu \nu}=\operatorname{diag}(1,-1,-1,-1)$ é a métrica quadridimensional de Minkowski. Também é valida a seguinte relação

$$
g_{\mu \nu} \equiv e^{-2 k|y|} \eta_{\mu \nu}
$$

A métrica (2.3) tem a propriedade particular que quando considerarmos as distâncias infinitesimais, ou seja os intervalos 5-dimensionais nas branas de planck $\left(\left.d s^{2}\right|_{y=L}\right)$ e $\mathrm{TeV}\left(\left.d s^{2}\right|_{y=0}\right)$ dado um mesmo intervalo 4-dimensional $d x_{\mu} d x^{\mu}$, isto produz a seguinte relação

$$
\left.d s^{2}\right|_{y=0}=\left.e^{-2 k L} d s^{2}\right|_{y=L} .
$$

Portanto, temos conseguido uma variação exponencial [15] das escalas através da dimensão extra, esta propriedade de escala terá consequências importantes quando um campo encontra-se sob uma brana. Para ver isto vamos considerar o campo de Higgs localizado na brana $\mathrm{TeV}$, então este campo tem sua dinâmica em $y=L$, portanto temos

$$
S_{H}=\int d^{4} x \int_{0}^{\pi R} d y \sqrt{g} \delta(y-L)\left[g^{\mu \nu} \partial_{\mu} H^{\dagger} \partial_{\nu} H-\lambda\left(|H|^{2}-v_{0}^{2}\right)^{2}\right]
$$

onde $g=\left|\operatorname{det}\left(g_{M, N}\right)\right|$, de (2.3) obtemos

$$
g=e^{-8 k|y|},
$$

usando (2.7) em (2.6), e depois de fazer a integral em $y$, a ação fica como

$$
S_{H}=\int d^{4} x\left[e^{-2 k L} \eta^{\mu \nu} \partial_{\mu} H^{\dagger} \partial_{\nu} H-e^{-4 k \pi R} \lambda\left(|H|^{2}-v_{0}^{2}\right)^{2}\right],
$$


agora absorvemos o fator $e^{-k L}$ no campo do Higgs, fazendo $e^{-k \pi R} H \rightarrow H$, para que campo $H$ fique canonicamente normalizado, então obtemos uma ação quadridimensional

$$
S_{H}=\int d^{4} x\left[\eta^{\mu \nu} \partial_{\mu} H^{\dagger} \partial_{\nu} H-\lambda\left(|H|^{2}-e^{-2 k L} v_{0}^{2}\right)^{2}\right]
$$

onde o valor esperado de vácuo efetivo é $v \equiv e^{-k \pi R} v_{0}$. Então temos uma solução ao problema da hierarquia do Modelo Padrão, porque se o campo do Higgs tem o VEV $v_{0}$ da ordem da escala de Planck, o VEV efetivo poderá ser da ordem da escala TeV. Se $k L \simeq 12$, a escala $\mathrm{TeV}$ é gerada dinâmicamente a partir da escala de Planck, isto é a massa física do campo de Higgs tem que aparecer nesta escala da energia. Então resolver o problema da hierarquia de gauge no Modelo Padrão implica estabilizar o comprimento da dimensão extra ${ }^{2}$, o que será abordado no próximo capítulo.

Agora, com o objetivo de resolver o problema da hierarquia das massas dos férmions, estudaremos este campo no bulk (espaço na dimensão y) e também vai nos interessar estudar os campos de gauge, especificamente estudaremos o modos de Kaluza-Klein em teorias $\mathrm{AdS}_{5}$. Isto será a ferramenta para entendermos a desconstrução de uma teoria 5-dimensional.

\subsubsection{Bósons de gauge na bulk}

Consideremos um campo de gauge 5-dimensional $A_{M}$ com a liberdade de se propagar na bulk. Sem perda de generalidade trataremos o caso abeliano $U(1)$ livre. Então a ação é dada por

$$
S_{A}=\int d^{5} x \sqrt{g}\left[-\frac{1}{4 g_{5}^{2}} F_{M N} F^{M N}\right]
$$

\footnotetext{
${ }^{2}$ Isto é conseguido depois de estudarmos o mecanismo de Goldberger-Wise
} 
onde $g_{5}$ é o acoplamento de gauge 5-dimensional $F_{M N}=\partial_{M} A_{N}-\partial_{N} A_{M}$. A ação (2.10) que pode ser simplificada a

$$
\begin{aligned}
S_{5}^{A} & =\int d^{5} x \sqrt{g}\left[-\frac{1}{4 g_{5}^{2}} g^{M O} g^{N P} F_{M N} F_{O P}\right], \\
& =\int d^{4} x \int_{0}^{L} d y \sqrt{g}\left[-\frac{1}{4 g_{5}^{2}} e^{4 k y} F_{\mu \nu} F^{\mu \nu}+\frac{1}{2 g_{5}^{2}} e^{2 k y}\left(F_{5 \mu}\right)^{2}\right], \\
& =\int d^{4} x \int_{0}^{L} d y\left[-\frac{1}{4 g_{5}^{2}} F_{\mu \nu} F^{\mu \nu}+\frac{1}{2 g_{5}^{2}} e^{-2 k y}\left(\partial_{5} A_{\mu}-\partial_{\mu} A_{5}\right)^{2}\right] .
\end{aligned}
$$

É importante notar que $F_{5 \mu}^{2}$ contém a mistura entre $A \mu$ e $A_{5}$, que pode ser eliminado trabalhando no gauge em que $A_{5}=0$ e impomos que $\partial_{\mu} A^{\mu}=0$. (gauge de Lorentz).

Agora achamos a equação de movimento, ou seja

$$
\delta S_{5}^{A}=-\frac{1}{4 g_{5}^{2}} \int d^{4} x \int_{0}^{L} d y\left[4 \partial^{\sigma} A^{\rho} \partial_{\sigma} \delta A_{\rho}-4 \partial^{\rho} A^{\sigma} \partial_{\sigma} \delta A_{\rho}+4 e^{-2 k y} \partial_{5} A_{\mu} \partial_{5} \delta A^{\mu}\right]
$$

Integramos por partes, cancelando os termos de superfície quadridimensionais obtemos

$$
\begin{aligned}
\delta S_{5}^{A}= & -\frac{1}{4 g_{5}^{2}} \int d^{4} x \int_{0}^{L} d y\left[4 \partial^{\sigma} A^{\rho} \partial_{\sigma} \delta A_{\rho}-4 \partial^{\rho} A^{\sigma} \partial_{\sigma} \delta A_{\rho}+4 e^{-2 k y} \partial_{5} A_{\mu} \partial_{5} \delta A^{\mu}\right] \\
= & -\frac{1}{g_{5}^{2}} \int d^{4} x \int_{0}^{L} d y\left[-\partial_{\sigma} \partial^{\sigma} A^{\mu} \delta A_{\mu}+\partial_{\sigma} \partial^{\mu} A^{\sigma} \delta A_{\mu}\right. \\
& \left.+\partial_{5}\left(e^{-2 k y} \partial_{5} A^{\mu} \delta A_{\mu}\right)-\partial_{5}\left(e^{-2 k y} \partial_{5} A^{\mu}\right) \delta A_{\mu}\right]
\end{aligned}
$$

de onde a equação de movimento é 


$$
\eta^{\mu \rho} \eta^{\nu \sigma} \partial_{\mu} F_{\rho \sigma}+\eta^{\nu \sigma} \partial_{5}\left(e^{-2 k y} \partial_{5} A_{\sigma}\right)=0
$$

com a condição que

$$
\left.\left(\delta A^{\mu} \partial_{5} A_{\mu}\right)\right|_{0, \pi R}=0
$$

onde podemos escolher as condições de fronteira de Neumann $\left.\partial_{5} A_{\mu}\right|_{0, \pi R}=0$ ou de Dirichlet $\left.A_{\mu}\right|_{0, \pi R}=0$.

Agora vamos introduzir a expansão em modos de Kaluza-Klein $A_{\mu}^{(n)}\left(x^{\nu}\right)$,

$$
A_{\mu}\left(x^{\nu}, y\right)=\sum_{n=0}^{\infty} A_{\mu}^{(n)}\left(x^{\nu}\right) f_{A}^{(n)}(y)
$$

onde $A_{\mu}^{(n)}\left(x^{\nu}\right)$ satisfaz a equação de Proca

$$
\eta^{\mu \rho} \partial_{\mu} F_{\rho \sigma}^{(n)}=m_{n}^{2} A_{\sigma}^{(n)} .
$$

Ao substituirmos (2.17) e (2.18) em (2.15) obtemos

$$
-\partial_{5}\left(e^{-2 k y} \partial_{5} f_{A}^{(n)}\right)=m_{n}^{2} f_{A}^{(n)},
$$

junto com a relação de ortonormalização

$$
\frac{1}{g_{5}^{2}} \int_{0}^{\pi R} d y f_{A}^{(n)} f_{A}^{(m)}=\delta_{n m}
$$

A seguir vamos mostrar a solução para o modo sem massa $m_{0}$ (modo zero), de $(2.19)$

$$
\partial_{5}\left(e^{-2 k y} \partial_{5} f_{A}^{(0)}\right)=0,
$$

de onde 


$$
f_{A}^{(0)}(y)=c_{0}^{(0)}+c_{1}^{(0)} e^{2 k y},
$$

onde $c_{1}^{(0)}, c_{2}^{(0)}$ são constantes a determinar. Só tem solução quando a condição de Neumann é imposta junto com (2.20) e obtemos

$$
f_{A}^{(0)}(y)=\frac{1}{\sqrt{\pi R}}
$$

então o modo zero do bóson de gauge é deslocalizado. O fato da função de onda do modo zero do bóson de gauge ser constante reflete o fato que a invariância de gauge do modo zero é mantida.

E para os estados massivos temos

$$
f^{(n)}(y)=\frac{e^{k y}}{N_{n}}\left[J_{1}\left(\frac{m_{n} e^{k y}}{k}\right)+\beta\left(m_{n}\right) Y_{1}\left(\frac{m_{n} e^{k y}}{k}\right)\right],
$$

onde $J_{1}$ e $Y_{1}$ são funções de Bessel de primeira e segunda ordem respectivamente. Sendo que $\beta\left(m_{n}\right)$ e $m_{n}$ podem ser encontradas a partir das condições de contorno impostas acima e $N_{n}$ é o coeficiente de normalização obtido de (2.20), para simplificar o cálculo de $N_{n}$ vamos utilizarmos o limite em que $m_{n} \ll k$ e $k R \gg 1$.

Nesse limite, obtemos que

$$
N_{n} \approx \frac{e^{\pi k R} \pi R}{g_{5}^{2} \sqrt{2 \pi k R}} J_{1}\left(\frac{m_{n} e^{\pi k R}}{k}\right) \approx \frac{e^{\pi k R / 2}}{g_{5}^{2}} \sqrt{\frac{R}{m_{n}}} .
$$

Utilizando o fato de que $A\left(x^{\mu}, y\right)$ é par sob a transformação $Z_{2}$, então de acordo com a expansão (2.17) $\beta\left(m_{n}\right)$ satisfaz

$$
\beta\left(m_{n}\right)=-\frac{J_{0}\left(\frac{m_{n}}{k}\right)}{Y_{0}\left(\frac{m_{n}}{k}\right)},
$$


e no limite em que $m_{n} \ll k$ e $k R \gg 1[16]$

$$
m_{n} \simeq\left(n-\frac{1}{4}\right) \pi k e^{-\pi k R}, \quad n>0
$$

No contexto da teoria efetiva concluímos que os bósons de gauge quadridimensionais têm massas crescentes a cada modo, que começam em $m_{1} \simeq 2,4 k e^{-\pi k R} \simeq \mathcal{O}(1) \mathrm{TeV}[17$, $18]$.

\subsubsection{Férmions na bulk}

A dinâmica dos campos fermiônicos 5-dimensionais com a liberdade de se propagar na bulk ${ }^{3}$ está inclusa na seguinte ação

$$
S_{5}^{f}=\int d^{4} x \int_{0}^{\pi R} d y \sqrt{g}\left[i \bar{\Psi} \Gamma^{M} \nabla_{M} \Psi+m_{\Psi} \bar{\Psi} \Psi\right]
$$

onde $\Gamma^{M}$ são as matrizes definidas segundo $\Gamma^{M}=E_{A}^{M} \gamma^{A}$, com $\gamma_{A}$ que satisfaz a álgebra de Clifford definida no espaço plano. $E_{A}^{M}$ é o inverso do vielbein $\left(e_{M}^{A}\right)$. O vielbein faz mudar os índices do espaço tempo curvo aos índices do espaço tangente (plano), e é definido pela relação:

$$
g_{M N}(x)=e_{M}^{a}(x) e_{N}^{b}(x) \eta_{a b}
$$

Para a métrica de Randall-Sundrum de (2.29) obtemos que

$$
E_{M}^{A}=\operatorname{diag}\left(e^{k y}, e^{k y}, e^{k y}, e^{k y}, 1\right) .
$$

\footnotetext{
${ }^{3}$ Semelhante aos bósons 5 dimensionais da seção anterior
} 
$\nabla_{M}$ é a derivada covariante dada por

$$
\nabla_{M}=\partial_{M}+\omega_{M},
$$

sendo que a conexão de spin é dada por

$$
\omega_{M}=\frac{i}{2} \mathcal{J}_{A B} \omega_{M}^{A B}
$$

onde $\mathcal{J}_{A B}=-\frac{i}{4}\left[\gamma_{A}, \gamma_{B}\right] . \quad \mathrm{E} \omega_{M}^{A}{ }_{B}=E_{R}^{A} \Gamma_{M S}^{R} E_{B}^{S}-E_{B}^{R} \partial_{M} E_{S}^{A}$, com $\Gamma_{M S}^{R}$ sendo os símbolos de Christoffel ${ }^{4}$. A conexão de spin é

$$
\omega_{M}=\left(\frac{k}{2} e^{-k y} \gamma_{\mu} \gamma_{5}, 0\right)
$$

Então a ação para os férmions pode ser simplificada a

$$
\begin{aligned}
S_{5}^{f}=\int d^{4} x \int d y & \left\{e^{-3 k y}\left(\bar{\Psi}_{L} i \gamma^{\mu} \partial_{\mu} \Psi_{L}+\bar{\Psi}_{R} i \gamma^{\mu} \partial_{\mu} \Psi_{R}\right)\right. \\
& +e^{-4 k y} m_{\psi}\left(\bar{\Psi}_{L} \Psi_{R}+\bar{\Psi}_{R} \Psi_{L}\right) \\
& -\frac{1}{2}\left[\bar{\Psi}_{L}\left(e^{-4 k y} \partial_{5}+\partial_{5} e^{-4 k y}\right) \Psi_{R}\right. \\
& \left.\left.-\bar{\Psi}_{R}\left(e^{-4 k y} \partial_{5}+\partial_{5} e^{-4 k y}\right) \Psi_{L}\right]\right\} .
\end{aligned}
$$

Podemos escrever a massa $5 D$ do férmion em unidades da curvatura vezes um coeficiente de ordem $\mathcal{O}(1) \mathrm{c}, m_{\Psi}=c k$. Dado que o campo do férmion tem que se transformar sob $Z_{2}$ faz com que vamos ter que escolher $\Psi(-y)= \pm \gamma_{5} \Psi(y)$. Depois da decomposição quiral o modo zero $\Psi_{R}$ é par para $\Psi(-y)=+\gamma_{5} \Psi(y)$ e $\Psi_{L}$ é par para $\Psi(-y)=+\gamma_{5} \Psi(y)$, então escrevemos os férmions quirais numa expansão de modos de Kaluza-Klein

\footnotetext{
${ }^{4}$ Os símbolos de Christoffel para a métrica (2.3) são mostrados no Apêndice A
} 


$$
\Psi_{L, R} \equiv \frac{e^{\frac{3}{2} k y}}{\sqrt{\pi R}} \sum_{n=0}^{\infty} h_{L, R}^{(n)}(y) \psi_{L, R}^{(n)}\left(x^{\mu}\right),
$$

junto com a condição de ortonormalização

$$
\frac{1}{\pi R} \int_{0}^{\pi R} h_{L, R}^{*(n)}(y) h_{L, R}^{(m)}(y) d y=\delta^{n m}
$$

Semelhante ao caso dos bósons, obtemos neste caso equações de movimento para os $h_{L, R}^{(n)}$ dada por

$$
\partial_{5}^{2} h_{L, R}^{(n)}-2 k \partial_{5} h_{L, R}^{(n)}+m_{n}^{2} e^{2 k y} h_{L, R}^{(n)}+\left(\frac{3}{4}-c(c \pm 1)\right) k^{2} h_{L, R}^{(n)}=0
$$

com " + " para os modos de mão-esquerda e " - " para os modos de mão-direita. As soluções de (2.37) são dadas por [20]

$$
h_{L, R}^{(n)}(y)=\frac{e^{k y}}{N_{n}}\left[J_{\left|c \pm \frac{1}{2}\right|}\left(\frac{m_{n} e^{k y}}{k}\right)+b_{\left|c \pm \frac{1}{2}\right|} Y_{\left|c \pm \frac{1}{2}\right|}\left(\frac{m_{n} e^{k y}}{k}\right)\right] .
$$

onde $b_{\left|c \pm \frac{1}{2}\right|}$ pode ser determinado das condições de contorno e $N_{n}$ é o coeficiente de normalização que pode ser obtido de (2.36).

Em particular as soluções canonicamente normalizadas para o modo zero dos férmions são caracterizadas por uma exponencial dependem do parâmetro $c$, ou seja

$$
\begin{aligned}
& h_{L}^{(0)}(y)=\sqrt{\frac{k\left(1-2 c_{L}\right)}{\left[e^{k \pi R\left(1-2 c_{L}\right)}-1\right]}} e^{k y\left(\frac{1}{2}-c_{L}\right)}, \\
& h_{R}^{(0)}(y)=\sqrt{\frac{k\left(1+2 c_{R}\right)}{\left[e^{k \pi R\left(1+2 c_{R}\right)}-1\right]}} e^{k y\left(\frac{1}{2}+c_{R}\right)} .
\end{aligned}
$$

As Figuras (2.2) e (2.3) mostra a localização dos férmions depende do valor do parâmetro $c_{L, R}$.

Portanto concluímos mencionando que a função de onda modo zero esquerdo esta 

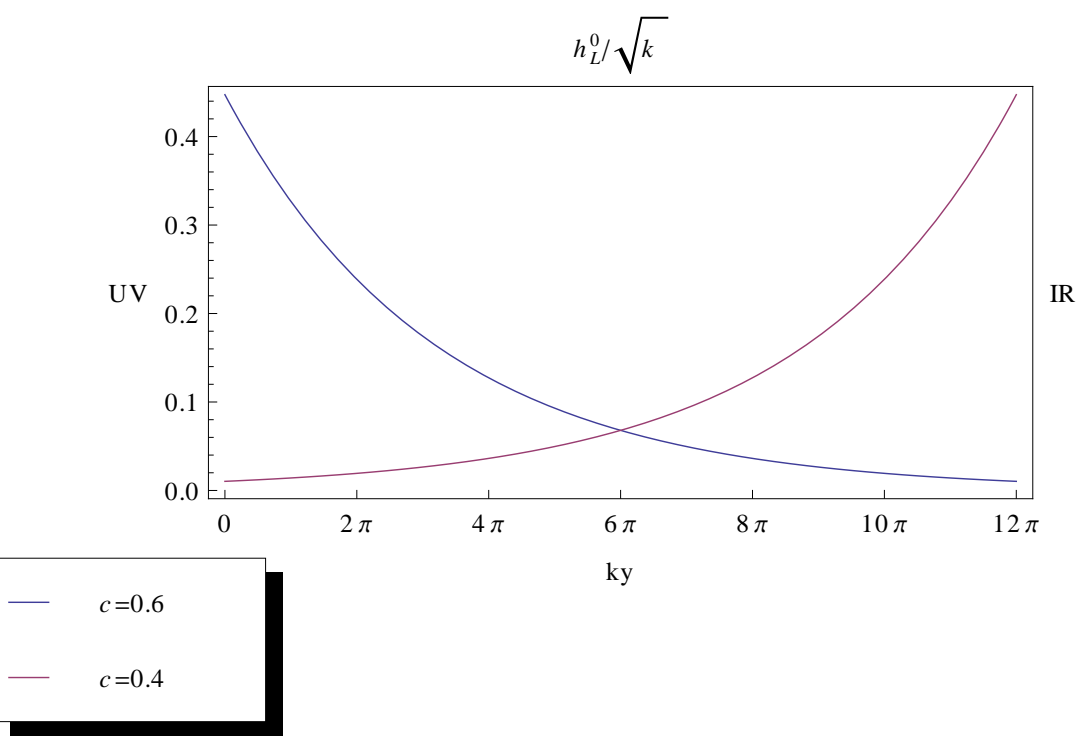

ky

Figura 2.2: Função $h_{L}^{(0)}(y) / \sqrt{k}$ para valores de $c=0.4,0.6$ em função de $k y$.
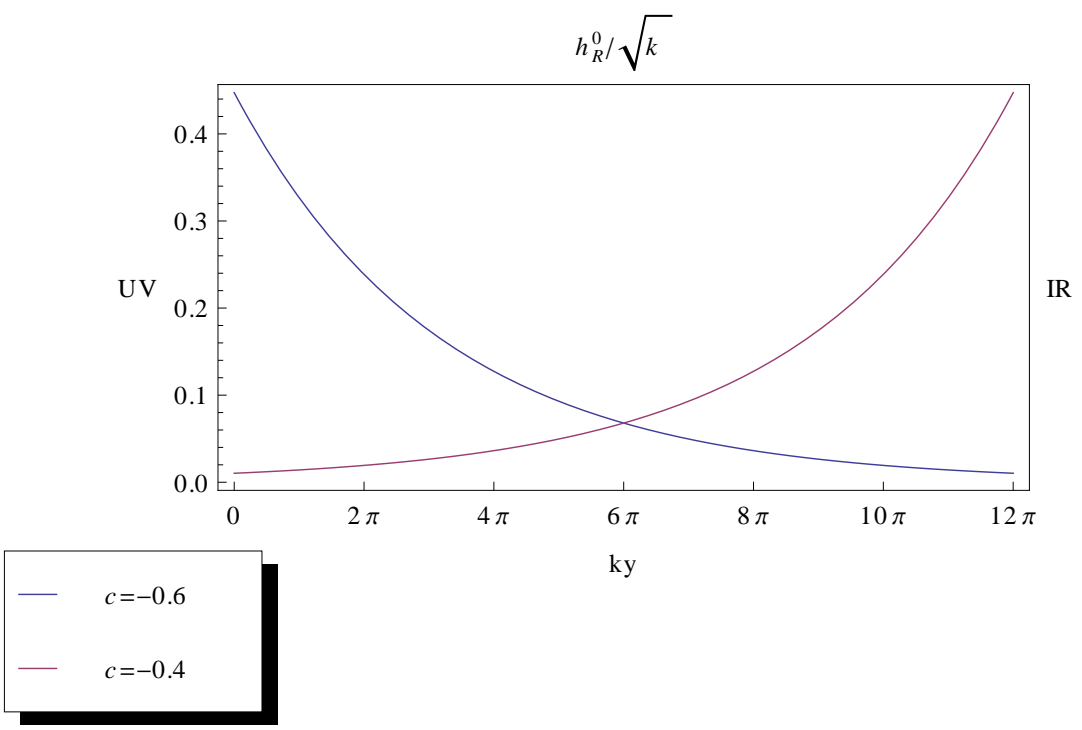

ky

Figura 2.3: Função $h_{R}^{(0)}(y) / \sqrt{k}$ para valores de $c=-0.6,-0.4$ em função de $k y$.

localizada na brana UV quando $c_{L}>1 / 2$. Também temos que a função modo zero direito está localizado na brana UV quando $c_{R}<-1 / 2$ o modo zero de mão-esquerda está localizado próximo à brana UV para e perto da brana IR para $c_{L}<1 / 2$. Já o modo zero de mão-direita estará localizado próximo à brana UV se e perto da brana IR para $c_{R}>-1 / 2$. Esse comportamento pode ser visto nos gráficos abaixo, onde 
plotamos as funções de onda $h_{L, R}^{(0)}(y) / \sqrt{k}$ para diferentes valores de $c$ em função de $k y$, sendo que $0 \leq k y \leq 12 \pi^{5}$.

Os férmions perto do IR terão maior acoplamento com o Higgs, portanto maior acoplamento de Yukawa $4 D$ e maior massa. Acontece o contrario com os férmions perto da brana UV eles têm menor acoplamento de Yukawa $4 D$, portanto menor massa.

Vemos que o fato de permitir que os férmions se propagarem na bulk faz com que os modos zero estejam localizados nas branas (UV, IR) o que realiza dinamicamente a hierarquia das massas dos férmions.

\subsection{Desconstrução de uma Teoria de Gauge}

Sabemos que as teorias $\mathrm{AdS}_{5}$ resolvem os problemas da hierarquia e das massas dos férmions. Porém elas são teorias não renormalizáveis, por isso interessa obter uma maior universo de teorias que resolvem as enormes hierarquias. Vamos começar considerando uma ação de gauge 5-dimensional contínua para o caso abeliano $(U(1)) A_{M}$. A extensão para o caso não abeliano $(S U(n))$ tem uma abordagem muito similar. Trabalhando em teorias $\mathrm{AdS}_{5}$, onde a dimensão extra é compactificada no orbifold $S_{1} / Z_{2}$ com $-L \leq$ $y \leq L$ e a métrica é

$$
d s^{2}=e^{-2 k|y|} \eta_{\mu \nu} d x^{\mu} d x^{\nu}-d y^{2},
$$

onde $k$ é a curvatura $\mathrm{AdS}_{5}$, a açaõ em consideração para bósons de gauge foi obtida em (2.11), e junto com o gauge em que $A_{5}=0$ temos

$$
S_{5}^{A}=\int d^{4} x \int_{0}^{L} d y\left[-\frac{1}{4 g_{5}^{2}} F_{\mu \nu} F^{\mu \nu}+\frac{1}{2 g_{5}^{2}} e^{-2 k y}\left(\partial_{5} A_{\mu}\right)^{2}\right]
$$

\footnotetext{
${ }^{5}$ Notar que $k R \approx 12$ resolve o problema da hierarquia entre a escala d Planck e TeV
} 
onde $g_{5}$ é o acoplamento de gauge em 5 dimensões. A seguir vamos discretizar a dimensão compacta, com espaçamento $\ell$ nesta variável. Então a ação contínua (2.41) ficará agora

$$
S_{5}^{A}=\ell \int d^{4} x \sum_{j=0}^{N}\left[-\frac{1}{4 g_{5 j}^{2}}\left(F_{\mu \nu}^{j} F^{j \mu \nu}\right)+\frac{1}{2 g_{5 j}^{2}} e^{-2 k \ell j}\left(\frac{A_{\mu}^{j}-A_{\mu}^{j-1}}{\ell}\right)^{2}\right]
$$

Essa ação corresponde à de uma teoria quadridimensional correspondente a um grupo de gauge produto da seguinte forma:

$$
G=G_{0} \times G_{1} \times \ldots \times G_{N-1} \times G_{N} .
$$

Aqui $G_{j}=S U(n)_{j}$ é um grupo de simetria da teoria de Yang-Mills ${ }^{6}$ com normalização $\operatorname{tr}\left[t_{j}^{a} t_{j}^{b}\right]=\frac{1}{2} \delta^{a b}$, onde $j=0,1, \ldots, N$. Para cada grupo $G_{j}$ corresponde um campo de gauge $A_{\mu, a}^{\prime j}$, onde $a=1,2, \ldots, n^{2}-1$. Também vamos precisar dos campos escalares de ligação $\left(\Phi_{j}\right)$ que se transformam como $(n, \bar{n})$ sob a representação bifundamental dos grupos $G_{j-1} \times G_{j}$, vamos considerar o modelo quiver bosônico 4D [20] ver Figura(2.4).

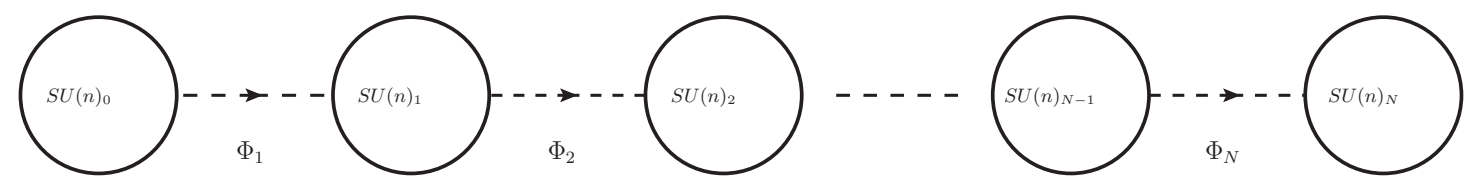

Figura 2.4: Diagrama quiver para uma cadeia linear, onde os círculos representam os grupos de gauge não abeliano $S U(n)_{j}$ ou abeliano $U(n)_{j}$ e $\left(\Phi_{j}\right)$ são os chamados campos de ligação.

Os círculos representam os grupos de gauge e as linhas os campos de ligação $\left(\Phi_{j}\right)$. Uma flecha saindo de um círculo indica que o campo de ligação transforma-se sob a representação fundamental daquele grupo e uma flecha entrando em um círculo significa que o campo de ligação transforma-se sob a representação anti-fundamental daquele

\footnotetext{
${ }^{6}$ No caso mais simples seria de uma teoria gauge abeliana onde $G_{j}=U(n)_{j}$.
} 
grupo. A ação 4D com as considerações de acima é dada por

$$
S_{4}^{A}=\int d^{4} x \sum_{j=0}^{N}\left\{-\frac{1}{2} \operatorname{Tr}\left(F_{\mu \nu}^{\prime j} F^{\prime j \mu \nu}\right)+\operatorname{Tr}\left[\left(D_{\mu} \Phi_{j}\right)^{\dagger}\left(D^{\mu} \Phi_{j}\right)\right]-V(\Phi)\right\}
$$

onde $F_{\mu \nu}^{\prime j}=F_{\mu \nu}^{\prime j a} t^{a}$ é o tensor intensidade do campo é dado por

$$
F_{\mu \nu}^{\prime j a}=\partial_{\mu} A_{\nu}^{\prime j a}-\partial_{\nu} A_{\mu}^{\prime j a}+g_{j} f^{j a b c} A_{\mu}^{\prime j b} A_{\nu}^{\prime j c},
$$

onde os $f_{j}^{a b c}$ são as constantes de estrutura, dadas por $\left[T_{j}^{a}, T_{j}^{b}\right]=i f_{j}^{a b c} T_{j}^{c}$, sendo os $T_{j}^{a}$ os geradores do grupo $S U(n)_{j}$. A derivada covariante $D_{\mu} \Phi_{j}$ é escrita como

$$
D_{\mu} \Phi_{j}=\partial_{\mu} \Phi_{j}+i g_{j-1} A_{\mu, j-1 a}^{\prime} T_{j-1}^{a} \Phi_{j}-i g_{j} \Phi_{j} A_{\mu, j a}^{\prime} T_{j}^{a} .
$$

A baixas energias podemos usar o modelo $\sigma$ linear. Isto é, o potencial é minimizado através dos valores esperados de vácuo $(\mathrm{VEV})$ dos campos de ligação, sendo que $\left\langle\Phi_{j}\right\rangle=$ $v_{j} \mathbf{1}_{m} / \sqrt{2}$ a fim de parametrizarmos os campos de ligação assim

$$
\Phi_{j}=\frac{\left[v_{j}+\sigma_{j}(x)\right]}{\sqrt{2}} e^{\left(i \xi_{j, a}(x) T_{j}^{a}\right) / v_{j}}
$$

onde os campos $\sigma_{j}$ são as flutuações de vácuo na direção radial, os campos $\xi_{j, a}$ são os bósons de Nambu-Goldstone da quebra de $S U(n)_{j-1} \times S U(n)_{j}$, além disso vamos considerar que os campos $\sigma_{j}$ adquirem massas suficientemente grandes, então estes não se propagam neste limite. Portanto caímos no modelo $\sigma$ não linear onde a parametrização vai como

$$
\Phi_{j}=\frac{v_{j}}{\sqrt{2}} e^{\left(i \xi_{j, a}(x) T_{j}^{a}\right) / v_{j}}
$$

o que resulta em $N \times\left(n^{2}-1\right)$ bósons de Nambu-Goldstone. Na ação (2.44) estamos 
considerando que todos os grupos dos $(N+1)$ sítios são iguais. Assim temos que $T_{j}^{a}=T_{j-1}^{a} \equiv T^{a} \quad \forall j$, com $a=1, \ldots, n^{2}-1$. Adicionalmente vamos considerar que os acoplamentos do grupo de gauge do sítio $j$ e $j-1$ os quais são avaliados no VEV correspondente serão iguais a $g_{j}$, então o produto das derivadas covariantes dos campos $\Phi_{j}$ em (2.44) covariantes junto com a substituição de (2.48) ficará

$$
\begin{aligned}
\left(D_{\mu} \Phi_{j}\right)^{\dagger}\left(D^{\mu} \Phi_{j}\right) & =\frac{1}{2}\left(\partial_{\mu} \xi_{j, a} T_{j}^{a}\right)\left(\partial^{\mu} \xi_{j, a} T_{j}^{a}\right)+\frac{v_{j}^{2} g_{j}^{2}}{2}\left(A_{\mu, j a}^{\prime} T_{j}^{a}-A_{\mu, j-1 a}^{\prime} T_{j-1}^{a}\right)^{2} \\
& +v_{j} g_{j} \partial^{\mu} \xi_{j} T_{j}^{a}\left(A_{\mu, j-1 a}^{\prime} T_{j-1}^{a}-A_{\mu, j a}^{\prime} T_{j}^{a}\right) \\
& =\frac{1}{2}\left[\partial_{\mu} \xi_{j, a} T_{j}^{a}+v_{j} g_{j}\left(A_{\mu, j-1 a}^{\prime} T_{j-1}^{a}-A_{\mu, j a}^{\prime} T_{j}^{a}\right)\right]^{2}
\end{aligned}
$$

Procuramos agora um gauge onde seja possível eliminar os bósons de NambuGoldstone da teoria. Veja que através da transformação de gauge

$$
\begin{gathered}
A_{\mu, j a}^{\prime} T^{a} \rightarrow A_{\mu, j a}^{\prime} T^{a}-\partial_{\mu} \alpha_{j, a}(x) T^{a}, \\
A_{\mu, j-1 a}^{\prime} T^{a} \rightarrow A_{\mu, j-1 a}^{\prime} T^{a},
\end{gathered}
$$

e temos a liberdade de escolher $\alpha_{j, a}(x) T^{a} \equiv-\xi_{j, a}(x) T^{a} / v_{j}$ (gauge unitário), de onde teremos que

$$
A_{\mu, j-1 a}^{\prime} T^{a}-A_{\mu, j a}^{\prime} T^{a} \rightarrow A_{\mu, j-1 a}^{\prime} T^{a}-A_{\mu, j a}^{\prime} T^{a}-\frac{1}{v_{j} g_{j}} \xi_{j, a}(x) T^{a} .
$$

A seguir substituímos (2.52) em (2.49),

$$
\left(D_{\mu} \Phi_{j}\right)^{\dagger}\left(D^{\mu} \Phi_{j}\right) \rightarrow \frac{1}{2} v_{j}^{2} g_{j}^{2}\left(A_{\mu, j-1 a}^{\prime} T^{a}-A_{\mu, j a}^{\prime} T^{a}\right)^{2} .
$$

Então os bósons de Nambu-Goldstone são "absorvidos"pelos bósons de gauge $A_{\mu, j}^{\prime}$ que se tornam massivos. A seguir substituímos (2.53) na ação (2.44) 


$$
S_{4}^{A}=\int d^{4} x \sum_{j=0}^{N}\left[-\frac{1}{4} \operatorname{Tr}\left(F_{\mu \nu, j}^{\prime} F_{j}^{\prime \mu \nu}\right)+\frac{1}{2} \operatorname{Tr}\left[v_{j} g_{j}\left(A_{\mu, j-1}^{\prime}-A_{\mu, j}^{\prime}\right)\right]^{2}\right],
$$

com o objetivo de obter a equivalência com a teoria de gauge $5 D$ da equação $(2.42)$, discretizada. Para isso devemos escolher o VEV de cada campo $\Phi_{j}$ em cada sítio como

$$
v_{j}=v q^{j},
$$

com $0<q<1$, onde os VEVs dos campos de ligação decrescem do sítio 0 até o sítio $N$ e a reestrição de trabalhar no caso em que os grupos $G_{j}=U(1)_{j}, \operatorname{logo}(2.54)$ terá a seguinte forma ${ }^{7}$

$$
S_{4}^{A}=\int d^{4} x \sum_{j=0}^{N}\left[-\frac{1}{4}\left(F_{\mu \nu, j} F_{j}^{\mu \nu}\right)+\frac{1}{2}\left[v_{j} g_{j}\left(A_{\mu, j-1}-A_{\mu, j}\right)\right]^{2}\right] .
$$

Conclusão: a teoria $4 D$ com campos de ligação $\Phi_{j}$ é equivalente a uma teoria $5 D$ discretizada.

A seguir vamos obter o espectro de massas dos bósons de gauge da teoria (2.56), identificamos só os termos de massa que vem dados por

$$
\mathcal{L}_{\text {massa }}=\frac{1}{2} \sum_{j=0}^{N}\left[v_{j} g_{j}\left(A_{\mu, j-1}-A_{\mu, j}\right)\right]^{2}
$$

Vamos considerar agora que cada acoplamento $g_{j}=g$, e que na base $\left(A_{\mu, 0}, A_{\mu, 1}, \ldots, A_{\mu, N}\right)$, a matriz de massa para os bósons de gauge [20], usando (2.55) é dada por

\footnotetext{
7 os campos $A_{\mu, j a}^{\prime}$ e $A_{\mu, j}$ são os campos de gauge que correspondem a $S U(N)$ e $U(N)$ respectivamente
} 


$$
M^{2}=g^{2} v^{2}\left(\begin{array}{ccccccc}
q^{2} & -q^{2} & 0 & 0 & \ldots & 0 & 0 \\
-q^{2} & q^{2}+q^{4} & -q^{4} & 0 & \ldots & 0 & 0 \\
0 & -q^{4} & q^{4}+q^{6} & -q^{6} & \ldots & 0 & 0 \\
\vdots & \vdots & \vdots & \vdots & \ldots & \vdots & \vdots \\
0 & 0 & 0 & 0 & \ldots & q^{2(N-1)}+q^{2 N} & -q^{2 N} \\
0 & 0 & 0 & 0 & \ldots & -q^{2 N} & q^{2 N}
\end{array}\right)
$$

Para diagonalizar esta matriz vamos definir uma rotação ortonormal entre as bases $A_{\mu, n}$ e $A_{\mu,(n)}$ através da mudança de bases

$$
A_{\mu, j}=\sum_{n=0}^{N} f_{j, n} A_{\mu,(n)},
$$

de tão maneira que se cumpra que na base dos auto-estados de massa $A_{\mu,(n)}$, onde $\mathcal{L}_{\text {massa }}$ pode ser escrito como

$$
\mathcal{L}_{\text {massa }}=\sum_{n=0} \frac{1}{2} m_{n}^{2} A_{\mu,(n)}^{2}
$$

Então para que a matriz (2.58) seja diagonal os coeficientes $f_{j, n}$ devem satisfazer a seguinte equação de diferenças [21]

$$
\left[q+q^{-1}-q^{-1}\left(x_{n} q^{-j}\right)^{2}\right] f_{j, n}-q f_{j+1, n}-q^{-1} f_{j-1, n}=0
$$

onde

$$
x_{n}^{2} \equiv m_{n}^{2} / g^{2} v^{2}
$$

A mesma equação para os coeficientes $f_{j, n}$ pode ser obtida das equações de movimento para os campos $A_{\mu, j}$, usando a lagrangiana dada por

$$
\mathcal{L}_{A}=\sum_{j=0}^{N}\left\{-\frac{1}{4} F_{\mu \nu, j} F_{j}^{\mu \nu}+\frac{g^{2}}{2} \operatorname{Tr}\left[v_{j}\left(A_{\mu, j-1}-A_{\mu, j}\right)\right]^{2}\right\}
$$

e usando as equações de Euler-Lagrange 


$$
\frac{\partial \mathcal{L}}{\partial A_{\nu, j}}-\partial_{\mu}\left(\frac{\partial \mathcal{L}}{\partial\left(\partial_{\mu} A_{\nu, j}\right)}\right)=0
$$

obtemos a seguinte equação

$$
\left(\partial^{2} A_{j}^{\nu}-\partial^{\nu} \partial_{\mu} A_{j}^{\mu}\right)+g^{2} v_{j}^{2}\left(A_{j}^{\nu}-A_{j-1}^{\nu}\right)+g^{2} v_{j+1}^{2}\left(A_{j}^{\nu}-A_{j+1}^{\nu}\right)=0 .
$$

Agora vamos usar o gauge de Lorentz, $\partial_{\mu} A_{j}^{\mu}=0$ e substituindo (2.59) em (2.65) temos

$$
f_{j, n} \partial^{2} A_{\nu,(n)}+g^{2} v^{2} q^{2 j}\left[\left(1+q^{2}\right) f_{j, n}-f_{j-1, n}-q^{2} f_{j+1, n}\right] A_{\nu,(n)}=0 .
$$

Impondo que $A_{\nu,(n)}$ satisfaz a equação de Proca, ou seja temos também a seguinte relação proveniente daquela imposição para o campo $A_{\nu,(n)}$

$$
\partial^{2} A_{\nu, n}=-m_{n}^{2} A_{\nu, n}
$$

substituímos (2.67) e usando a definição (2.62) em (2.66) segue que obtemos a mesma equação que (2.61)

$$
\left[q+q^{-1}-q^{-1}\left(x_{n} q^{-j}\right)^{2}\right] f_{j, n}-q f_{j+1, n}-q^{-1} f_{j-1, n}=0,
$$

junto com as condições de contorno

$$
f_{0, n}=f_{-1, n}, \quad f_{N, n}=f_{N+1, n},
$$

com a condição de normalização 


$$
\frac{1}{g^{2}} \sum_{j=0}^{N} f_{j, n}^{2}=1
$$

vamos nós concentrar agora no modo zero, $n=0, m_{0}=0$.

A equação (2.68) ficará

$$
\left[q+q^{-1}\right] f_{j, 0}-q f_{j+1,0}-q^{-1} f_{j-1,0}=0,
$$

de onde para $j=0$

$$
\left[q+q^{-1}\right] f_{0,0}-q f_{1,0}-q^{-1} f_{-1,0}=0
$$

e (2.69) conseguimos

$$
f_{0,0}=f_{1,0}
$$

fazendo iterações para os outros $j$ s temos que

$$
f_{0,0}=f_{1,0}=f_{2,0}=\ldots=f_{N, 0} .
$$

Se agora usamos a condição de normalização (2.70) obtemos que

$$
f_{j, 0}=\frac{g}{\sqrt{N+1}}
$$

Isto é, as componentes do modo zero são iguais em todos os sítios. Isto é análogo ao que acontece na teoria $\mathrm{AdS}_{5}$, onde os modos zero dos bósons estão deslocalizados.

Para os modos massivos a equação (2.68) tem solução para os modos massivos [21], definimos as seguintes variáveis 


$$
\begin{gathered}
t[j]=x_{n} q^{-j}, \\
F(t[j])=q^{j} f_{j, n},
\end{gathered}
$$

e substituindo (2.75) e (2.76) na equação de q-diferenças (2.68) obtemos

$$
\left(q+q^{-1}-q^{-1} t^{2}\right) F(t)-F\left(t q^{-1}\right)-F(t q)=0 .
$$

A equação (2.77) é um caso especial da equação de Hahn-Exton [21, 22] com soluções que são as chamadas funções de q-Bessel. As soluções mais gerais podem ser escritas como

$$
F(t)=A J_{1}\left(t ; q^{2}\right)+B Y_{1}\left(t ; q^{2}\right),
$$

onde em geral $J_{\nu}\left(t ; q^{2}\right)$ e $Y_{1}\left(t ; q^{2}\right)$ são as funções de q-Bessel é de q- Neumann ${ }^{8}$ respectivamente definidas a seguir [21]

$$
J_{\nu}(t ; q)=t^{\nu} \frac{\left(q^{\nu+1} ; q\right)_{\infty}}{(q ; q)_{\infty}} \sum_{i=0}^{\infty} \frac{(-1)^{i} q^{i(i+1) / 2}}{\left(q^{\nu+1} ; q\right)_{i}(q ; q)_{i}} t^{2 i}
$$

com os fatores $(y ; q)_{i}$ são definidos por

$$
(y ; q)_{k}= \begin{cases}1 & \text { se } k=0 \\ \prod_{n=0}^{k-1}\left(1-y q^{n}\right) & \text { se } k \geq 1\end{cases}
$$

para $y \in \mathbb{C}, \quad i \in \mathbb{Z}_{+}=\{0,1,2, \ldots\}$ e $(y ; q)_{\infty} \equiv \lim _{i \rightarrow \infty}(y ; q)_{i}$.

entretanto

$$
Y_{\nu}(t ; q)=\frac{\Gamma_{q}(\nu) \Gamma_{q}(1-\nu)}{\pi} q^{-\nu^{2} / 2}\left[\cos (\pi \nu) q^{\nu / 2} J_{\nu}(t ; q)-J_{-\nu}\left(t q^{-\nu / 2} ; q\right)\right],
$$

\footnotetext{
${ }^{8}$ no limite $\mathrm{q} \rightarrow 1^{-}$obtemos as funções ordinárias contínuas de Bessel e Neumann [21]
} 
onde a função $\Gamma_{q}(\nu)$ definida por

$$
\Gamma_{q}(\nu)=\frac{(q ; q)_{\infty}}{\left(q^{\nu} ; q\right)_{\infty}}(1-q)^{1-\nu}
$$

Usando (2.76), (2.77) e as condições de contorno (2.69) é possível encontrar os coeficientes $f_{j, n}$ a menos de uma constante $N_{n}$ que pode ser obtida da condição de normalização $(2.70)$

$$
f_{j, n}=N_{n} q^{-j}\left[Y_{0}\left(x_{n} ; q^{2}\right) J_{1}\left(x_{n} q^{-j} ; q^{2}\right)-J_{0}\left(x_{n} ; q^{2}\right) Y_{1}\left(x_{n} q^{-j} ; q^{2}\right)\right] .
$$

Adicionalmente o espectro de massas é obtido da equação

$$
J_{0}\left(x_{n} ; q^{2}\right) Y_{0}\left(q^{-(N+1)} x_{n} ; q^{2}\right)-Y_{0}\left(x_{n} ; q^{2}\right) J_{0}\left(q^{-(N+1)} ; q^{2}\right)=0 .
$$

No limite do contínuo, quando $q \rightarrow 1^{-}$, estes coeficientes (2.83) coincidem com as funções de onda(perfis 5-dimensional) dos modos excitados $(n)$ dos bósons de gauge na dimensão extra. Então com a desconstrução de uma teoria de gauge 5-dimensional se pode gerar uma teoria correspondente em 4-dimensões.

Para fazer evidente isto vamos reescalar os campos de gauge da ação (2.56) assim

$$
g_{j} A_{\mu, j} \rightarrow A_{\mu, j}
$$

nossa ação (2.54) fica

$$
S_{4}^{A}=\int d^{4} x \sum_{j=0}^{N}\left[-\frac{1}{4 g_{j}}\left(F_{\mu \nu, j} F_{j}^{\mu \nu}\right)+\frac{1}{2}\left[v_{j}\left(A_{\mu, j-1}-A_{\mu, j}\right)\right]^{2}\right],
$$

Podemos ver a equivalência de ambas teorias estabelecendo o dicionário entre a teoria de gauge 5-dimensional discretizada (2.42) com a teoria de gauge puramente quadridimensional (2.86) ver tabela 2.1. Assim mesmo identificamos o sítios zero e N 
como as branas $U V$ e $I R$ respectivamente.

\begin{tabular}{ccc} 
Teoria com 4 dimensões & & Teoria com 5 dimensões \\
\hline$v_{j}$ & $\leftrightarrow$ & $\frac{e^{-k \ell j}}{\ell}$ \\
$\frac{1}{g_{j}^{2}}$ & $\leftrightarrow$ & $\frac{\ell}{g_{5 j}^{2}}$
\end{tabular}

Tabela 2.1: Dicionário entre a teoria de gauge desconstruída 4-dimensional e a teoria de gauge com uma dimensão extra curva discretizada 5 dimensional.

Sabemos que as teorias $\mathrm{AdS}_{5}$ resolvem o problema da hierarquia de gauge para $k L \approx 37[14,16]$

A teoria desconstruída no limite para a teoria contínua $\left(\mathrm{AdS}_{5}\right)$ é obtida quando $N \rightarrow \infty$ e $\ell \rightarrow 0$ com $N \ell \rightarrow L$, onde $L$ é o tamanho da dimensão extra e $\ell$ é o espaçamento da rede.

Então temos que

$$
k N \ell \sim 37 .
$$

Vamos ver que acontece com $v_{1}$ em relação com $v_{N}$, no limite do contínuo

$v_{1}=\frac{e^{-k \ell}}{\ell}$ e $v_{N}=\frac{e^{-k \ell N}}{\ell}$ então, convenientemente formamos o cociente

$$
\frac{v_{1}}{v_{N}}=\frac{1}{e^{-37}}
$$

temos que $v_{1} \gg v_{N}$ que se corresponde a expressão encontrada ao considerar o campo de Higgs localizado perto da brana $\mathrm{TeV} v \equiv e^{-k \pi R} v_{0}$, onde mencionamos que $v$ e $v_{0}$ eram da ordem das escalas de Planck e TeV respectivamente.

Então a enorme hierarquia de escalas pode ser gerada pelas teorias puramente $4 D$. Chamaremos a essas teorias $4 D$ Teorias Quiver de Hierarquia Completa $(T Q H C)$. 


\subsection{Desconstrução de uma Teoria que inclui Férmions}

Nesta seção vamos incluir os férmions na teoria. Considerando o mesmo cenário da dimensão extra curva que a seção anterior, a ação fermiônica 5-dimensional pode-se escrever na forma seguinte

$$
S_{5}^{f}=\int d^{4} x \int_{0}^{L} d y\left\{e^{-3 k y} \bar{\Psi} i \gamma_{\mu} \partial^{\mu} \Psi+e^{-4 k y} m_{\Psi} \bar{\Psi} \Psi-e^{-4 k y} \bar{\Psi} \gamma_{5} \overleftrightarrow{\partial_{5}} \Psi\right\}
$$

onde a massa do férmion de Dirac tem dada em unidades da curvatura dada por $m_{\Psi} \equiv$ $c k$ e $\overleftrightarrow{\partial_{5}} \equiv \frac{1}{2}\left(\overrightarrow{\partial_{5}}-\overleftarrow{\partial_{5}}\right)$

A ação (2.88) pode ser re-escrita como

$$
\begin{aligned}
S_{5}^{f}=\int d^{4} x \int_{0}^{L} d y & \left\{e^{-3 k y / 2} \bar{\Psi}\left(i \gamma_{\mu} \partial^{\mu}+e^{-k y} m_{\Psi}\right) e^{-3 k y / 2} \Psi\right. \\
& -\frac{e^{-k y}}{2}\left[e^{-3 k y / 2} \bar{\Psi} \gamma_{5} \partial_{5}\left(e^{-3 k y / 2} \Psi\right)\right. \\
& \left.\left.-\partial_{5}\left(e^{-3 k y / 2} \bar{\Psi}\right) \gamma_{5}\left(e^{-3 k y / 2} \Psi\right)\right]\right\},
\end{aligned}
$$

agora absorvemos o fator $e^{-3 k y / 2}$ no campo do férmion ${ }^{9}$ fazendo $e^{-3 k y / 2} \Psi \rightarrow \Psi$. A ação (2.89) pode ser discretizada, assim como para o caso dos bósons de gauge.

\footnotetext{
${ }^{9}$ Notar que o campo 5 -dimensional do férmion $\Psi$ tem dimensão de massa 2
} 
Obetemos,

$$
\begin{aligned}
S_{5}^{f}=\int d^{4} x \sum_{j=0}^{N} \ell & \left\{\bar{\Psi}_{j}\left(i \gamma_{\mu} \partial^{\mu}+e^{-k \ell j} m_{\Psi}\right) \Psi_{j}\right. \\
& \left.-\frac{e^{-k \ell j}}{2 \ell}\left(\bar{\Psi}_{j} \gamma_{5} \Psi_{j+1}-\bar{\Psi}_{j+1} \gamma_{5} \Psi_{j}\right)\right\} .
\end{aligned}
$$

Redefinindo o campo do férmion $\ell^{1 / 2} \Psi_{j} \rightarrow \psi_{j}$ para que ele esteja canonicamente normalizado, assim mesmo usando a projeção $\psi_{L, j ; R, j} \equiv \frac{1}{2}\left(1 \mp \gamma_{5}\right) \psi_{j}$ a $(2.90)$ é simplificada como

$$
\begin{aligned}
S_{5}^{f}=\int d^{4} x \sum_{j=0}^{N} & \left\{\bar{\psi}_{L, j} i \partial \psi_{L, j}+\bar{\psi}_{R, j} i \partial \psi_{R, j}\right. \\
& +e^{-k \ell j} m_{\Psi} \bar{\psi}_{j}\left(\bar{\psi}_{R, j} \psi_{L, j}+\bar{\psi}_{L, j} \psi_{R, j}\right) \\
& \left.+\frac{e^{-k \ell j}}{2 \ell}\left(\bar{\psi}_{R, j} \psi_{L, j+1}-\bar{\psi}_{L, j} \psi_{R, j+1}+\text { h.c. }\right)\right\} .
\end{aligned}
$$

A ação discretizada (2.91) tem o problema de dobrar o espectro de massas dos férmions [23], isto é o chamado problema de duplicação dos férmions [24], este problema pode ser resolvido usando o termo de Wilson

$$
S_{\text {Wilson }}=\eta \ell \int d^{4} x \int_{0}^{L} d y \sqrt{g} \bar{\Psi}\left(\partial_{5}\right)^{2} \Psi
$$

onde $\eta$ é um coeficiente arbitrário, notar que este termo vai para zero no limite do contínuo $(\ell \rightarrow 0)$. A discretização da ação de Wilson é

$$
S_{\text {Wilson }}=\eta \int d^{4} x \sum_{j=0}^{N} \frac{e^{-k \ell j}}{\ell}\left\{\bar{\psi}_{L, j} \psi_{R, j+1}+\bar{\psi}_{R, j+1} \psi_{L, j+1}-2 \bar{\psi}_{L, j} \psi_{R, j}+\text { h.c. }\right\} \text {. }
$$

$$
\text { Somando (2.91) e (2.93) obtemos }
$$




$$
\begin{aligned}
S_{5}^{f}+S_{\text {Wilson }}=\int d^{4} x \sum_{j=0}^{N} & \left\{\bar{\psi}_{L, j} i \not \partial \psi_{L, j}+\bar{\psi}_{R, j} i \not \partial \psi_{R, j}+e^{-k \ell j}\left(m_{\Psi}-\frac{2 \eta}{\ell}\right) \bar{\psi}_{j} \psi_{j}\right. \\
+ & {\left[\left(\eta-\frac{1}{2}\right) \frac{e^{-k \ell j}}{\ell} \bar{\psi}_{L, j} \psi_{R, j+1}\right.} \\
+ & \left.\left.\left(\eta+\frac{1}{2}\right) \frac{e^{-k \ell j}}{\ell} \bar{\psi}_{R, j} \psi_{L, j+1}+\text { h.c. }\right]\right\}
\end{aligned}
$$

Em (2.94) a escolha de $\eta= \pm 1 / 2$ vai resultar na eliminação de um dos modos zero. Se nós escolhermos $\eta=1 / 2$ que fixa a direção de salto (hopping direction), nós obteremos

$$
\begin{aligned}
S_{5}^{f}+S_{\text {Wilson }}=\int d^{4} x \sum_{j=0}^{N}\left\{\bar{\psi}_{L, j} i \not \partial \psi_{L, j}+\bar{\psi}_{R, j} i \not \partial \psi_{R, j}+\frac{e^{-k \ell j}}{\ell}(c k \ell-1) \bar{\psi}_{j} \psi_{j}\right. \\
\left.+\frac{e^{-k \ell j}}{\ell}\left(\bar{\psi}_{R, j} \psi_{L, j+1}+\text { h.c. }\right)\right\} .
\end{aligned}
$$

A seguir vamos escrever a teoria fermiônica quadridimensional ou $T Q H C$ que inclui os férmions. A ação neste caso é dada por

$$
\begin{aligned}
S_{4}=\int d^{4} x \sum_{j=0}^{N} & \left\{-\frac{1}{2} \operatorname{Tr}\left(F_{\mu \nu}^{\prime j} F^{\prime j \mu \nu}\right)+\operatorname{Tr}\left[\left(D_{\mu} \Phi_{j}\right)^{\dagger}\left(D^{\mu} \Phi_{j}\right)\right]\right. \\
& +\bar{\psi}_{L, j} i \not \partial \psi_{L, j}+\bar{\psi}_{R, j} i \not \partial \psi_{R, j}+\lambda \operatorname{Tr}\left(\bar{\psi}_{R, j-1} \Phi_{j} \psi_{L, j}+\text { h.c. }\right) \\
& \left.+\operatorname{Tr}\left(\mu_{j} \bar{\psi}_{L, j} \psi_{R, j}+\text { h.c. }\right)-V(\Phi)\right\},
\end{aligned}
$$

onde $F_{\mu \nu}^{\prime j}, D_{\mu} \Phi_{j}$ são dados por (2.45) e (2.46) respectivamente. A ação (2.96) é representada através do diagrama de quiver que inclui os férmions Figura(2.5).

Os círculos representam os grupos de gauge $S U(n)_{j}$, posteriormente os sítios zero e $\mathrm{N}$ serão identificados como as branas $U V$ e $I R$ respectivamente, as flechas verticais 


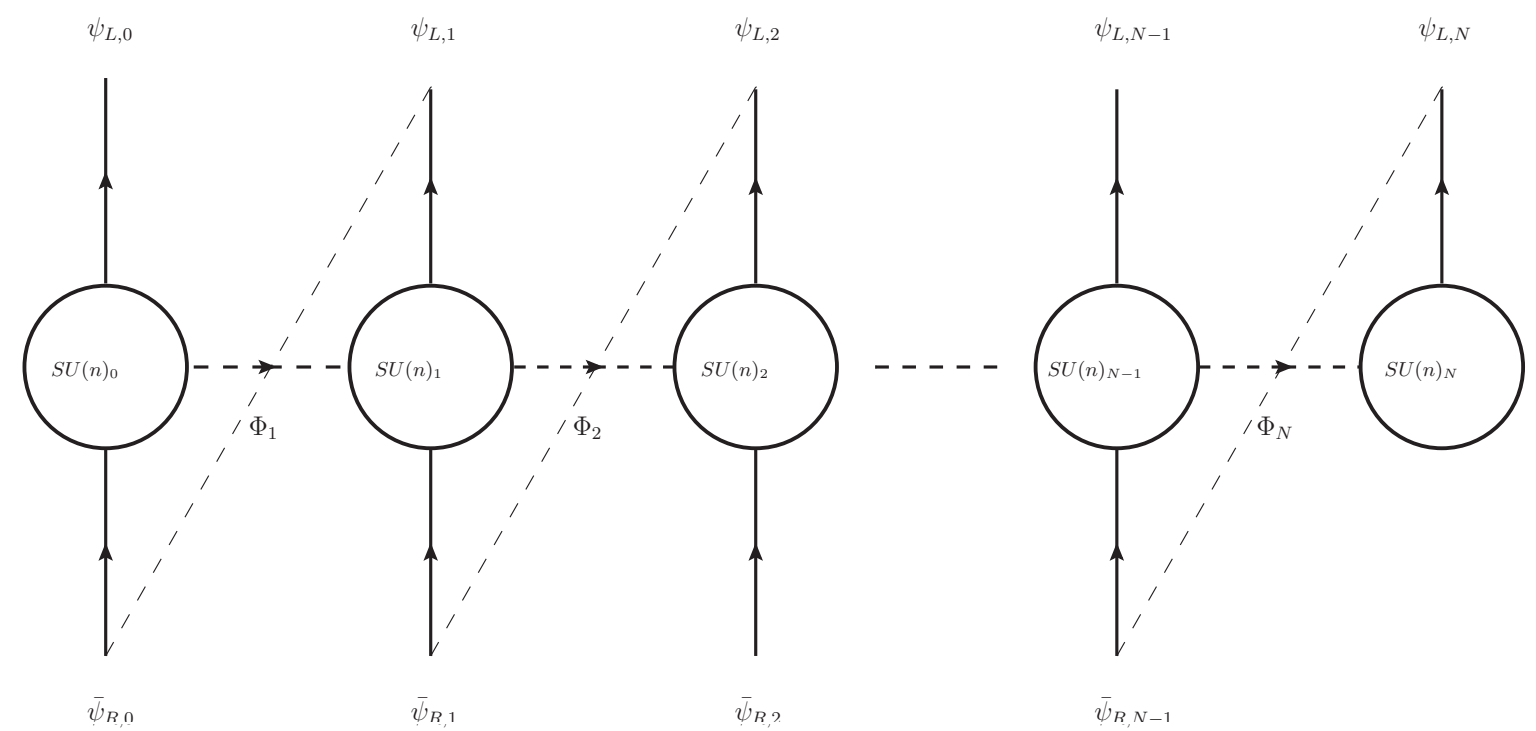

Figura 2.5: Diagrama de quiver para uma cadeia linear com círculos representando os grupos de gauge não abeliano $S U(n)_{j}$ ou abeliano $U(n)_{j}$ para cada sítio $j=0, \ldots, N$ incluindo férmions quirais e $\left(\Phi_{j}\right)$ são os chamados campos de ligação.

representam os férmions, assim uma seta que sai do círculo no sítio $j$ significa que o férmion quiral $\psi_{L, j}$ transforma-se sob a representação fundamental de $S U(n)_{j}$ pelo contrário uma seta entrando no círculo no sítio $j$ significa que o férmion quiral $\psi_{R, j}$ transforma-se sob a representação anti-fundamental de $S U(n)_{j}$. Os campos de ligação $\left(\Phi_{j}\right)$ transformam-se como,$(n, \bar{n})$ sob $S U(n)_{j-1} \times S U(n)_{j}$. Uma linha pontilhada entre os sítios $j-1$ e $j$ indica o acoplamento de Yukawa do campo de conexão $\Phi_{j}$ com os férmions quirais $\bar{\psi}_{R, j-1}$ e $\psi_{L, j}$.

As condições de contorno são tais que se o campo $\psi_{R, N}$ está ausente o modo zero vai ter de quiralidade esquerda. Para obter um modo zero de mão-direita em um diagrama quiver com a mesma direção de salto (hopping direction) temos que fazer $\psi_{L, 0}=0$.

A seguir vamos identificar o termo de massa dos férmions quirais da ação (2.96) lembrando que os valores esperados do vácuo (VEV) dos campos de ligação foram fixados na seção anterior $\left\langle\Phi_{j}\right\rangle=v_{j} \mathbf{1}_{m} / \sqrt{2}$. A lagrangiana fermiônica é 


$$
\begin{aligned}
S_{4}^{f}=\int d^{4} x \sum_{j=0}^{N} & \left\{\bar{\psi}_{L, j} i \not \partial \psi_{L, j}+\bar{\psi}_{R, j} i \not \partial \psi_{R, j}+\frac{\lambda v_{j}}{\sqrt{2}}\left(\bar{\psi}_{R, j-1} \psi_{L, j}+\text { h.c. }\right)\right. \\
& \left.+\left(\mu_{j} \bar{\psi}_{L, j} \psi_{R, j}+\text { h.c. }\right)\right\},
\end{aligned}
$$

sendo a lagrangiana de massa

$$
\mathcal{L}_{\text {massa }}=\sum_{j=0}^{N}\left[\frac{\lambda v_{j}}{\sqrt{2}}\left(\bar{\psi}_{R, j-1} \psi_{L, j}+\text { h.c. }\right)+\left(\mu_{j} \bar{\psi}_{L, j} \psi_{R, j}+\text { h.c. }\right)\right] .
$$

Identificando a base $\Psi_{L}=\left(\psi_{L, 0}, \psi_{L, 1}, \ldots, \psi_{L, N}\right)$, obtemos a matriz de massas ao quadrado na base $\Psi_{L}$ como

$M M^{T}=\left(\begin{array}{ccccccc}\mu_{0}^{2} & \frac{\lambda}{\sqrt{2}} \mu_{0} v_{1} & 0 & 0 & \cdots & 0 & 0 \\ \frac{\lambda}{\sqrt{2}} \mu_{0} v_{1} & \left(\frac{\lambda}{\sqrt{2}}\right)^{2} v_{1}^{2}+\mu_{1}^{2} & \frac{\lambda}{\sqrt{2}} \mu_{1} v_{2} & 0 & \cdots & 0 & 0 \\ 0 & \frac{\lambda}{\sqrt{2}} \mu_{1} v_{2} & \left(\frac{\lambda}{\sqrt{2}}\right)^{2} v_{2}^{2}+\mu_{2}^{2} & \frac{\lambda}{\sqrt{2}} \mu_{2} v_{3} & \cdots & 0 & 0 \\ \vdots & \vdots & \vdots & \vdots & \cdots & \vdots & \vdots \\ 0 & 0 & 0 & 0 & \cdots & \left(\frac{\lambda}{\sqrt{2}}\right)^{2} v_{N-1}^{2}+\mu_{N-1}^{2} & \frac{\lambda}{\sqrt{2}} \mu_{N-1} v_{N} \\ 0 & 0 & 0 & 0 & \cdots & \frac{\lambda}{\sqrt{2}} \mu_{N-1} v_{N} & \mu_{N}^{2}\end{array}\right)$

Assim como para o caso dos bósons de gauge, esta matriz pode ser diagonalizada formalmente fazendo a seguinte mudança de base

$$
\psi_{L, j}=\sum_{n=0}^{N} h_{j, n}^{L} \psi_{L,(n)}
$$

Similarmente, se considerarmos a base $\Psi_{R}=\left(\psi_{R, 0}, \psi_{R, 1}, \ldots, \psi_{R, N}\right)$, a mesma matriz pode também ser diagonalizada fazendo outra mudança de base 


$$
\psi_{R, j}=\sum_{n=0}^{N} h_{j, n}^{R} \psi_{R,(n)},
$$

onde $\psi_{L,(n)}$ e $\psi_{R,(n)}$ são os autoestados de massa por isso satisfazem a equação de Dirac

$$
\begin{aligned}
& i \not \partial \psi_{L,(n)}-m_{n} \psi_{R,(n)}=0, \\
& i \not \partial \psi_{R,(n)}-m_{n} \psi_{L,(n)}=0 .
\end{aligned}
$$

Por outro lado as equações de movimento podem ser obtidas de (2.97)

$$
\begin{aligned}
\operatorname{para} \bar{\psi}_{R, j}: & i \not \partial \psi_{R, j}+\frac{\lambda}{\sqrt{2}} v_{j} \psi_{L, j+1}+\mu_{j} \psi_{L, j}=0, e \\
\operatorname{para} \bar{\psi}_{L, j}: & i \not \partial \psi_{L, j}+\frac{\lambda}{\sqrt{2}} v_{j-1} \psi_{R, j-1}+\mu_{j} \psi_{R, j}=0,
\end{aligned}
$$

e usando (2.99), (2.100), (2.103) e (2.104) obtemos

$$
\begin{array}{r}
m_{n} h_{j, n}^{R}+\mu_{j} h_{j, n}^{L}+\frac{\lambda}{\sqrt{2}} v_{j} h_{j+1, n}^{L}=0, \\
m_{n} h_{j, n}^{L}+\mu_{j} h_{j, n}^{R}+\frac{\lambda}{\sqrt{2}} v_{j-1} h_{j-1, n}^{R}=0,
\end{array}
$$

onde as equações (2.105) e (2.106) estão acopladas. Depois de desacoplar elas obtemos 


$$
\begin{gathered}
\left(\mu_{j}^{2}+\frac{\lambda^{2}}{2} v_{j-1}^{2}-m_{n}^{2}\right) h_{j, n}^{L}+\frac{\lambda}{\sqrt{2}} \mu_{j} v_{j} h_{j+1, n}^{L}+\frac{\lambda}{\sqrt{2}} \mu_{j-1} v_{j-1} h_{j-1, n}^{L}=0 \\
\left(\mu_{j}^{2}+\frac{\lambda^{2}}{2} v_{j}^{2}-m_{n}^{2}\right) h_{j, n}^{R}+\frac{\lambda}{\sqrt{2}} \mu_{j+1} v_{j} h_{j+1, n}^{R}+\frac{\lambda}{\sqrt{2}} \mu_{j} v_{j-1} h_{j-1, n}^{R}=0
\end{gathered}
$$

Com a finalidade que o modelo quadridimensional desconstruído que inclui férmions seja equivalente a uma teoria fermiônica com uma dimensão extra curva no limite do contínuo, precisamos que além do obtido na tabela 2.1 seja satisfeito que

$$
\mu_{j}=-g v q^{c+j-1 / 2}, \quad \lambda=\sqrt{2} g, \quad \text { para } q \rightarrow 1^{-} .
$$

Agora usando (2.55), (2.62), (2.109) nas equações (2.107) e (2.108) obtemos

$$
\begin{aligned}
& {\left[q^{-\left(c+\frac{1}{2}\right)}+q^{\left(c+\frac{1}{2}\right)}-q^{-\left(c+\frac{1}{2}\right)}\left(x_{n} q^{-j}\right)^{2}\right] h_{j, n}^{L}-q h_{j+1, n}^{L}-q^{-1} h_{j-1, n}^{L}=0,} \\
& {\left[q^{-\left(c-\frac{1}{2}\right)}+q^{\left(c-\frac{1}{2}\right)}-q^{-\left(c-\frac{1}{2}\right)}\left(x_{n} q^{-j}\right)^{2}\right] h_{j, n}^{R}-q h_{j+1, n}^{R}-q^{-1} h_{j-1, n}^{R}=0 .}
\end{aligned}
$$

As equações (2.110) e (2.111) tem soluções ${ }^{10}$ que são as funções de q-Bessel e as funções de q-Neumann [22].

$$
\begin{aligned}
& h_{j, n}^{L}=N_{n}^{L} q^{-j}\left[J_{\left|c+\frac{1}{2}\right|}\left(x_{n} q^{-j} ; q^{2}\right)+b_{\left|c+\frac{1}{2}\right|}\left(x_{n} ; q^{2}\right) Y_{\left|c+\frac{1}{2}\right|}\left(x_{n} q^{-j} ; q^{2}\right)\right], \\
& h_{j, n}^{R}=N_{n}^{R} q^{-j}\left[J_{\left|c-\frac{1}{2}\right|}\left(x_{n} q^{-j} ; q^{2}\right)+b_{\left|c-\frac{1}{2}\right|}\left(x_{n} ; q^{2}\right) Y_{\left|c-\frac{1}{2}\right|}\left(x_{n} q^{-j} ; q^{2}\right)\right],
\end{aligned}
$$

\footnotetext{
${ }^{10}$ Semelhante à seção anterior para o caso dos bósons de gauge
} 
onde $N_{n}^{L}$ e $N_{n}^{R}$ são fatores de normalização. No limite do contínuo, é dizer $q \rightarrow 1^{-}$ as soluções (2.112) e (2.113) coincidem com as funções de onda 5-dimensional para os férmions na dimensão extra no cenário em consideração.

Para obtermos um modo zero quiral podemos impor como condição de contorno $h_{N, n}^{R}=0\left(\psi_{R, N}=0\right)$ para obter um modo zero de mão-esquerda, ou podemos impor $h_{0, n}^{L}=0\left(\psi_{L, 0}=0\right)$ para obter um modo zero de mão-direita.

Para o caso de um modo zero de mão-esquerda, usamos (2.105)

$$
\mu_{j} h_{j, 0}^{L}+\frac{\lambda}{\sqrt{2}} v_{j} h_{j+1,0}^{L}=0,
$$

que é equivalente a

$$
\frac{h_{j+1,0}^{L}}{h_{j, 0}^{L}}=q^{c_{L}-1 / 2} .
$$

Como $0<q<1$, para $c_{L}>1 / 2$ o modo zero de mão-esquerda estará "localizado" do lado esquerdo do diagrama Figura(2.5), próximo ao sítio zero, e para $c_{L}<1 / 2$ estará a direita do diagrama, próximo ao sítio $N$. Então podemos associar os sítios zero e $N$ com as escalas UV e IR respectivamente.

Alternativamente se considerarmos um modo zero de mão-direita, usamos $(2.106)$

$$
\mu_{j} h_{j, 0}^{R}+\frac{\lambda}{\sqrt{2}} v_{j-1} h_{j-1,0}^{R}=0
$$

que é equivalente a

$$
\frac{h_{j, 0}^{R}}{h_{j-1,0}^{R}}=q^{-\left(c_{R}+1 / 2\right)},
$$


consistentemente temos que para $c_{R}>-1 / 2$ o modo zero de quiralidade direita está no sítio $N$ (IR), por outro lado para $c_{R}<-1 / 2$ o modo zero está mais perto do sítio zero (UV).

Em ambos casos que a função de onda do férmion já seja de quiralidade direita ou esquerda, simplesmente quer dizer por exemplo, se o férmion está mais perto do sítio $\mathrm{N}$ associado com a escala (IR), este tem mais acople com o Higgs,

Analogamente se o férmion está localizado mais perto do sítio zero associado com a escala UV ele tem menor acople com o higgs [25].

As teorias $4 D$ terão características similares a $\mathrm{AdS}_{5}$, mas desse ponto de vista podemos dizer que têm diferente fenomenologia, em aspectos importantes da teoria. Mencionamos que as teorias $\mathrm{AdS}_{5}$ são um caso particular das $T Q H C$ no limite do contínuo. 


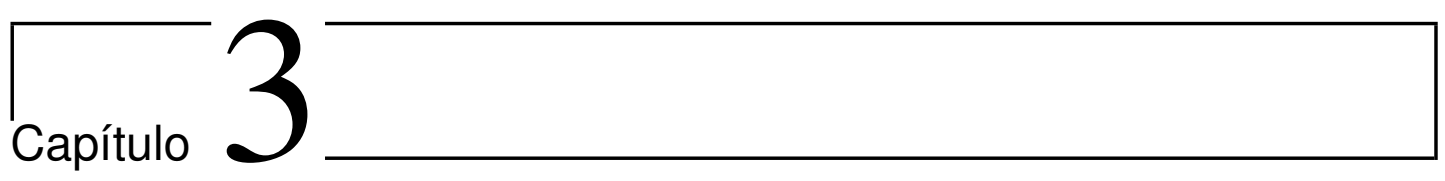

\section{Estabilização da Dimensão Extra em}

$\mathrm{AdS}_{5}$

Temos visto que considerar teorias com uma dimensão extra curva em $\mathrm{AdS}_{5}$, faz possível resolver o problema da hierarquia de gauge e da hierarquia da massa dos férmions. Já no capítulo anterior, ao implementar a desconstrução em $\mathrm{AdS}_{5}$, obtivemos uma teoria $4 D$ com todas as vantagens de $\mathrm{AdS}_{5}$ mais sem precisar de dimensões extras. No caso de teorias $\mathrm{AdS}_{5}$ é necessário assumir que existe um mecanismo que estabiliza a dimensão extra ( através da determinação dinâmica do raio da compactificação ). Isto garante que as teorias $\mathrm{AdS}_{5}$ sejam gravitacionalmente estáveis, e faz possível gerar a grande hierarquia entre as escalas UV e IR. Com o objetivo de entender a necessidade de introduzir um mecanismo de estabilização da dimensão extra, consideramos a métrica 5-dimensional, segundo

$$
d s^{2}=e^{\frac{-2 k|y| T(x)}{r_{c}}} g_{\mu \nu}(x) d x^{\mu} d x^{\nu}-\frac{T^{2}(x)}{r_{c}^{2}} d y^{2}
$$

onde $g_{\mu \nu}(x)$ é a métrica 4-dimensional, $T(x)$ é uma flutuação independente de 
$y$ conhecido como campo módulo ${ }^{1}$. Essa métrica vai ser a métrica RS no caso em que $g_{\mu \nu}$ seja plana (métrica de Minkowski) e que o VEV de $T(x)$ seja $\pi r_{c}=L$.

Vamos começar pela ação Einstein-Hilbert 5-dimensional considerando só o escalar da curvatura $R_{5}{ }^{2}$ (escalar de Ricci 5D) sem a constante cosmológica,

$$
S=-2 M^{3} \int d^{4} x d y \sqrt{\operatorname{det}\left(g_{M N}\right)} R_{5},
$$

que pode ser expressada em termos do escalar da curvatura ${ }^{3} R_{4}$ e $T(x)$, segundo

$$
\begin{aligned}
S= & 2 M^{3} \int d^{4} x d y \frac{\sqrt{-g} e^{\frac{-2 k|y| T}{r_{c}}}}{r_{c}}\left\{\frac{6 k|y|}{r_{c}} \partial_{\mu} T \partial^{\mu} T\right. \\
& \left.-\frac{6 k^{2}|y|^{2} T}{r_{c}^{2}} \partial_{\mu} T \partial^{\mu} T-T R_{4}-20 k^{2} T e^{\frac{-2 k|y| T}{r_{c}}}\right\} .
\end{aligned}
$$

Fazemos a integração em $y$, e considerando $-L \leq y \leq L \operatorname{com} L=\pi r_{c}$ e obtemos

$$
\begin{aligned}
S= & -\frac{2 M^{3}}{k} \int d^{4} x \sqrt{-g}\left(1-e^{-2 k \pi T}\right) R_{4}+\frac{12 M^{3}}{k} \int d^{4} x \sqrt{-g} k^{2} \pi^{2} e^{-2 k \pi T} \partial_{\mu} T \partial^{\mu} T \\
& -20 M^{3} k \int d^{4} x \sqrt{-g}\left(1-e^{-4 k \pi T}\right) .
\end{aligned}
$$

Re-arranjando a expressão de acima temos:

$$
\begin{aligned}
S= & -\frac{2 M^{3}}{k} \int d^{4} x \sqrt{-g}\left(1-e^{-2 k \pi T}\right) R_{4} \\
& +\frac{12 M^{3}}{k} \int d^{4} x \sqrt{-g} \partial_{\mu}\left(e^{-k \pi T}\right) \partial^{\mu}\left(e^{-k \pi T}\right) \\
& -20 M^{3} k \int d^{4} x \sqrt{-g}\left(1-e^{-4 k \pi T}\right) .
\end{aligned}
$$

\footnotetext{
${ }^{1} \mathrm{Na}$ literatura $\mathrm{T}$ é o modulus field

${ }^{2} \mathrm{O}$ escalar de curvatura $R_{5}$ é mostrado no Apêndice $\mathrm{B}$

${ }^{3}$ Os cálculos são mostrados no Apêndice B.
} 
Definimos $\varphi=f \exp (-k \pi T) \operatorname{com} f=\sqrt{24 M^{3} / k}$, e então (3.5) fica dada por

$$
\begin{aligned}
S= & -\frac{2 M^{3}}{k} \int d^{4} x \sqrt{-g}\left(1-(\varphi / f)^{2}\right) R_{4}+\frac{1}{2} \int d^{4} x \sqrt{-g} \partial_{\mu} \varphi \partial^{\mu} \varphi \\
& -20 M^{3} k \int d^{4} x \sqrt{-g}\left(1-(\varphi / f)^{4}\right) .
\end{aligned}
$$

Portanto temos um escalar quadridimensional sem massa [27]. A equação anterior não inclui uma dinâmica que faça estabilizar $\varphi$, ou seja o potencial que depende só do campo $\varphi$ é nulo, e não se pode obter um correspondente VEV para $T$ a não ser que seja nulo, mas isso seria quando $r_{c}=0$.

Vimos no capítulo anterior que se o valor de $k L \approx 36$, é possível gerar a escala TeV a partir da escala de Planck. Então, o problema da hierarquia pode ser resolvida se achamos um mecanismo que faz possível estabilizar o raio da dimensão extra. Na seguinte seção vamos ver a proposta de estabilização dada por Goldberger-Wise. Desejaríamos que existisse um mecanismo similar nas TQHC em $4 D$.

\subsection{Mecanismo de Goldberger-Wise}

Com o objetivo de estabilizar o raio da dimensão extra, Walter Goldberger e Mark Wise $[26,27]$ propuseram o mecanismo $G W$, que consiste em gerar um potencial efetivo para estabilizar a quantidade $L=\pi r_{c}$. Vamos considerar um campo escalar 5-dimensional na bulk de $\mathrm{AdS}_{5}$

$$
S_{\text {bulk }}=\frac{1}{2} \int d^{4} x \int_{-L}^{L} d y \sqrt{\operatorname{det}\left(g_{M N}\right)}\left(g^{A B} \partial_{A} \Phi \partial_{B} \Phi-m^{2} \Phi^{2}\right)
$$


onde $M, N, A, B=\mu, 5$, com termos de interação nas branas de Planck e TeV dadas pelas ações $S_{h}$ e $S_{v}$ respectivamente

$$
\begin{aligned}
& S_{h}=-\int d^{4} x \sqrt{-g_{h}} \lambda_{h}\left(\Phi^{2}-v_{h}^{2}\right)^{2}, \\
& S_{v}=-\int d^{4} x \sqrt{-g_{v}} \lambda_{v}\left(\Phi^{2}-v_{v}^{2}\right)^{2},
\end{aligned}
$$

onde $g_{h, v}=\left.\operatorname{det}\left(g_{M, N}\right)\right|_{y=0, L}$, também é simples notar que $\Phi, v_{v, h}$ têm dimensão de massa $3 / 2, \lambda_{v, h}$ tem dimensão de massa -2 e $m$ tem dimensão de massa 1.

A ação de $\Phi$ é dada por

$$
S_{G W}=S_{b u l k}+S_{h}+S_{v}
$$

e então a equação de movimento para o campo $\Phi$ é

$$
\begin{aligned}
0= & \partial_{\mu}\left(e^{-2 k|y|} \partial^{\mu} \Phi\right)-\partial_{y}\left(e^{-4 k|y|} \partial_{y} \Phi\right)+m^{2} e^{-4 k|y|} \Phi \\
& +4 e^{-4 k|y|} \lambda_{v} \Phi\left(\Phi^{2}-v_{v}^{2}\right) \delta(y-L)+4 e^{-4 k|y|} \lambda_{h} \Phi\left(\Phi^{2}-v_{h}^{2}\right) \delta(y),
\end{aligned}
$$

onde temos imposto que

$$
\left.e^{-4 k|y|}\left(\partial_{y} \Phi\right) \delta \Phi\right|_{y=-L} ^{L}=0
$$


Em geral o VEV do $\Phi$ depende de $y$, a equação (3.11) fica simplificada a

$$
\begin{aligned}
0= & -\partial_{y}\left(e^{-4 k|y|} \partial_{y}\langle\Phi\rangle\right)+m^{2} e^{-4 k|y|}\langle\Phi\rangle \\
& +4 e^{-4 k|y|} \lambda_{v}\langle\Phi\rangle\left(\langle\Phi\rangle^{2}-v_{v}^{2}\right) \delta(y-L)+4 e^{-4 k|y|} \lambda_{h}\langle\Phi\rangle\left(\langle\Phi\rangle^{2}-v_{h}^{2}\right) \delta(y),
\end{aligned}
$$

é uma equação diferencial (3.13) de segundo ordem em y cuja solução para $0 \leqslant$ $y \leqslant L$ é

$$
\langle\Phi\rangle=e^{2 k|y|}\left(A e^{\nu k|y|}+B e^{-\nu k|y|}\right)
$$

com $\nu=\sqrt{4+m^{2} / k^{2}}, A$ e $B$ são constantes a determinar.

Substituindo esta solução na ação(3.10), e depois de fazer a redução dimensional, obtemos o valor negativo de um potencial efetivo quadridimensional que depende de $L$,

$$
\begin{aligned}
S=\frac{1}{2} \int d^{4} x \int_{-L}^{L} d y & {\left[-A^{2}\left(8 k^{2}+4 k^{2} \sqrt{4+\frac{m^{2}}{k^{2}}}+2 m^{2}\right) e^{2 k|y| \nu}\right.} \\
& \left.-B^{2}\left(8 k^{2}-4 k^{2} \sqrt{4+\frac{m^{2}}{k^{2}}}+2 m^{2}\right) e^{-2 k|y| \nu}\right] .
\end{aligned}
$$

Portanto, o potencial é dado por

$$
\begin{aligned}
V= & k(\nu+2) A^{2}\left(e^{2 \nu k L}-1\right)+k(\nu-2) B^{2}\left(1-e^{-2 \nu k L}\right) \\
& +\lambda_{v} e^{-4 k L}\left(\Phi(L)^{2}-v_{v}^{2}\right)^{2}+\lambda_{h}\left(\Phi(0)^{2}-v_{h}^{2}\right)^{2} .
\end{aligned}
$$

Agora se considerarmos por simplicidade que $\lambda_{h}$ e $\lambda_{v}$ sejam parâmetros muitos grandes, teremos aproximadamente 


$$
\Phi(0)=v_{h}, \quad \Phi(L)=v_{v}
$$

Com (3.16) podemos achar os valores de $A$ e $B$ em (3.14),

$$
\begin{aligned}
A & =\frac{v_{h} e^{-2 \nu k L}-v_{v} e^{-(2+\nu) k L}}{e^{-2 \nu k L}-1}, \\
B & =\frac{v_{h}-v_{v} e^{-(2+\nu) k L}}{e^{-2 \nu k L}-1}
\end{aligned}
$$

Simplificando uma vez que as potências de $e^{-k L}$ são desprezíveis obtemos,

$$
\begin{aligned}
& A=v_{v} e^{-(2+\nu) k L}-v_{h} e^{-2 \nu k L}, \\
& B=v_{h}\left(1+e^{-2 \nu k L}\right)-v_{v} e^{-(2+\nu) k L} .
\end{aligned}
$$

Vamos supor também que $m / k \ll 1$, e portanto temos que $\nu=2+\epsilon$, onde $\epsilon \simeq m^{2} / 4 k^{2}$.

Agora ao considerar (3.20), (3.21), (3.17) e ignorando termos proporcionais a $\epsilon$ o potencial de (3.16) fica

$$
V=4 k e^{-4 k L}\left(v_{v}-v_{h} e^{-\epsilon k L}\right)^{2} .
$$

O potencial achado só depende do parâmetro livre que neste caso é $L=\pi r_{c}$. O mínimo de $V$ é obtido quando se satisfaz

$$
e^{\epsilon k L}=\frac{v_{h}}{v_{v}}
$$


o que é equivalente a ter

$$
k L=\frac{1}{\epsilon} \ln \left[\frac{v_{h}}{v_{v}}\right]
$$

de onde obtemos que

$$
k r_{c}=\left(\frac{4}{\pi}\right) \frac{k^{2}}{m^{2}} \ln \left[\frac{v_{h}}{v_{v}}\right] .
$$

Se considerarmos $\frac{v_{h}}{v_{v}}=1.5$ e $\frac{m}{k}=0.2$ obtemos $k r_{c} \simeq 12$, esta escolha não constitui um ajuste fino extremo.

Por outro lado, da seção anterior temos que o campo do radion $\varphi$ pode ser identificado no potencial (3.22) com a suposição que $T(x)=r_{c}+\delta T$, onde $\delta T \rightarrow 0$ para coincidir com a métrica RS. Então o potencial pode se re-escrever como

$$
V=\frac{k^{3}}{144 M^{6}} \varphi^{4}\left(v_{v}-v_{h}\left(\frac{\varphi}{f}\right)^{\epsilon}\right)^{2}
$$

O procedimento para minimizar $V$ é achar a primeira derivada do $\frac{\partial V}{\partial \varphi}=0$ e resolver para $\varphi$. Então obtemos

$$
\frac{\partial V}{\partial \varphi}=\frac{k^{3} \epsilon \varphi^{4}\left(\frac{\varphi}{f}\right)^{-1+\epsilon} v_{h}\left(-\left(\frac{\varphi}{f}\right)^{\epsilon} v_{h}+v_{v}\right)}{72 f M^{6}}+\frac{k^{3} \varphi^{3}\left(-\left(\frac{\varphi}{f}\right)^{\epsilon} v_{h}+v_{v}\right)^{2}}{36 M^{6}}
$$

de onde depois de resolver temos

$$
\frac{\langle\varphi\rangle}{f}=\left(\frac{v_{v}}{v_{h}}\right)^{1 / \epsilon}
$$

Agora massa para o campo $\varphi$ pode ser obtida a partir da segunda derivada do potencial 


$$
\begin{aligned}
\frac{\partial^{2} V}{\partial \varphi^{2}}= & \frac{k^{3} \epsilon^{2} \phi^{4}\left(\frac{\phi}{f}\right)^{-2+2 \epsilon} v_{h}^{2}}{72 f^{2} M^{6}}-\frac{k^{3}(-1+\epsilon) \epsilon \phi^{4}\left(\frac{\phi}{f}\right)^{-2+\epsilon} v_{h}\left(-\left(\frac{\phi}{f}\right)^{\epsilon} v_{h}+v_{v}\right)}{72 f^{2} M^{6}} \\
& -\frac{k^{3} \epsilon \phi^{3}\left(\frac{\phi}{f}\right)^{-1+\epsilon} v_{h}\left(-\left(\frac{\phi}{f}\right)^{\epsilon} v_{h}+v_{v}\right)}{9 f M^{6}}+\frac{k^{3} \phi^{2}\left(-\left(\frac{\phi}{f}\right)^{\epsilon} v_{h}+v_{v}\right)^{2}}{12 M^{6}} .
\end{aligned}
$$

Avaliando a segunda derivada obtida no VEV obtido em (3.27), obtemos uma massa para o campo $\varphi$ dada por

$$
m_{\varphi}^{2}=\left.\frac{\partial^{2} V}{\partial \varphi^{2}}\right|_{\varphi=\langle\varphi\rangle}=\frac{k^{2} v_{v}^{2}}{3 M^{3}} \epsilon^{2} e^{-2 k r_{c} \phi}
$$

Devemos notar que a massa do radion é ajustada hacia a brana $\mathrm{TeV}$ por meio do fator $e^{-k r_{c} \phi}$, só que também tem uma supressão devido ao fator $\epsilon$, então esta partícula tem massa menor do que a escala $\mathrm{TeV}$, e portanto é mais ligeira do que os outros modos de Kaluza-Klein.

O radion é o pseudo-bóson de Nambu-Goldstone da quebra da simetria conforme, que fixa a brana $I R$ já que uma vez que o mecanismo de $G W$ é introduzido, a ação efetiva obtida em (3.6) vai resultar numa massa para o campo do radion $\varphi$ o que faz quebrar a invariância de escala. Então o radion é o dílaton da quebra da simetria de escala ${ }^{4}$.

No capítulo seguinte vamos estudar as teorias $4 D T Q H C$ com o objetivo de saber se é possível ter um dílaton leve nessas teorias.

\footnotetext{
${ }^{4}$ Ver simetria de escala na Introdução.
} 
$\Gamma_{\text {Capítulo }} 4$

\section{O Dílaton em Teorias Quiver}

\subsection{Radion}

Temos mencionado no capítulo anterior que no contexto de teorias $\mathrm{AdS}_{5}$, a excitação do raio implica a existência de um escalar chamado de radion [28]. Uma vez que é imposto o mecanismo de estabilização da dimensão extra, isto garante que as teorias $\mathrm{AdS}_{5}$ sejam gravitacionalmente estáveis.

Vamos considerar agora teorias desconstruídas com a finalidade de mostrar que é possível a existência de um dílaton leve. No capítulo anterior ele foi o autêntico radion das Teorias $\mathrm{AdS}_{5}$. Vamos considerar uma teoria de gauge $4 D$ de simetria Abeliana ${ }^{1}$ com diagrama de quiver mostrada na Figura (4.1).

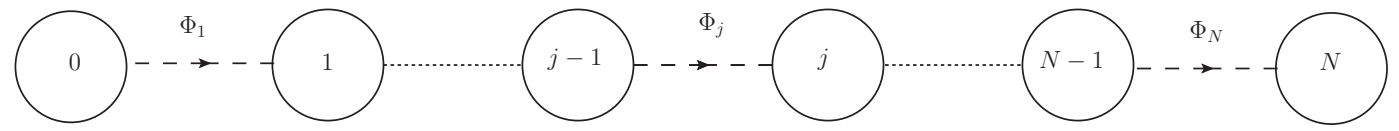

Figura 4.1: Diagrama de quiver para uma Teoria $4 D$ que inclui bósons de gauge.

Escrevemos a seguir a teoria $4 D$, que inclui só bósons de gauge com $N+1$ sítios e os campos de conexão $\Phi_{j}$ dada por

\footnotetext{
${ }^{1} \mathrm{O}$ pode-se optar pelo caso não Abeliano também.
} 


$$
S_{4}^{A}=\int d^{4} x\left\{-\frac{1}{4 g^{2}} \sum_{j=0}^{N}\left(F_{\mu \nu}^{j} F^{j \mu \nu}\right)+\sum_{j=1}^{N}\left[\left|\mathcal{D}_{\mu} \Phi_{j}\right|^{2}-V\left(\left|\Phi_{j}\right|^{2}\right)\right]\right\}
$$

onde

$$
\mathcal{D}_{\mu} \Phi_{j}=\partial_{\mu} \Phi_{j}+i A_{\mu, j-1} \Phi_{j}-i \Phi_{j} A_{\mu, j}
$$

Vamos parametrizamos o campo de conexão $\phi_{j}$ de acordo com o modelo $\sigma$ não linear temos

$$
\Phi_{j}=\frac{\left[v_{j}+h_{j}(x)\right]}{\sqrt{2}} e^{i \frac{G_{j}}{v_{j}}}
$$

Fazendo a consideração que os campos $h_{j}$ adquirem massas suficientemente grandes então a parametrização fica segundo

$$
\Phi_{j}=\frac{v_{j}}{\sqrt{2}} e^{i \frac{G_{j}}{v_{j}}}
$$

Podemos escrever o termo cinético para o campo $\Phi_{j}$, é dizer

$$
\begin{aligned}
\left|\mathcal{D}_{\mu} \Phi_{j}\right|^{2}=\mathcal{D}_{\mu}^{\dagger} \Phi_{j} \mathcal{D}_{\mu} \Phi_{j}= & \frac{1}{2} \partial_{\mu} G_{j} \partial^{\mu} G_{j}+\frac{v_{j}^{2}}{2}\left(A_{\mu, j}-A_{\mu, j-1}\right)^{2} \\
& +v_{j}\left(A_{\mu, j-1}-A_{\mu, j}\right) \partial^{\mu} G_{j}
\end{aligned}
$$

No gauge unitário, os bósons de Nambu-Goldstone são eliminados.

Ele é

$$
A_{\mu, j-1}-A_{\mu, j} \rightarrow A_{\mu, j-1}-A_{\mu, j}-\frac{1}{v_{j}} \partial_{\mu} G_{j}
$$


Então no gauge de acima o termo cinético (4.5) dos campos de ligação vai como

$$
\mathcal{D}_{\mu}^{\dagger} \Phi_{j} \mathcal{D}_{\mu} \Phi_{j}=\frac{v_{j}^{2}}{2}\left(A_{\mu, j}-A_{\mu, j-1}\right)^{2}
$$

Substituindo (4.7) em (4.1) , e considerando que o potencial é desprezível $V\left(\left|\Phi_{j}\right|^{2}\right)$ obtemos

$$
S_{4}^{A}=\int d^{4} x\left\{-\frac{1}{4 g^{2}} \sum_{j=0}^{N}\left(F_{\mu \nu}^{j} F^{j \mu \nu}\right)+\sum_{j=1}^{N}\left[\frac{v_{j}^{2}}{2}\left(A_{\mu, j}-A_{\mu, j-1}\right)^{2}\right]\right\} .
$$

A ação (4.8) não é invariante sob a transformação de escala, devido aos termos misturados de $A_{\mu, j} \operatorname{com} A_{\mu, j-1}$. As transformações de escala ${ }^{2}$ são tais que

$$
\alpha: x_{\mu} \rightarrow e^{-\alpha} x_{\mu}
$$

o campo bosônico vai se transformar, segundo

$$
\alpha: A_{\mu, j} \rightarrow e^{\alpha} A_{\mu, j}
$$

Mas podemos considerar que a simetria de escala é realizada, mas é espontaneamente quebrada [2]. Nesse caso a ação (4.8) vai ser invariante ao introduzir um campo $\sigma_{j}$ definido segundo

$$
v_{j} e^{\frac{\sigma_{j}}{v_{j}}}=\chi_{j}
$$

\footnotetext{
${ }^{2}$ Semelhante à simetria de escala mencionada na introdução
} 
Se o campo $\sigma_{j}$ se transforma de acordo com

$$
\sigma_{j} \rightarrow v_{j} \alpha+\sigma_{j}
$$

então que a ação (4.8) será invariante de escala. Podemos multiplicar com potências de $e^{\frac{\sigma_{j}}{v_{j}}}$ de acordo com [2] nas partes da ação que quebram a simetria de escala, então obtemos

$$
S_{4}^{A}=\int d^{4} x\left\{-\frac{1}{4 g^{2}} \sum_{j=0}^{N}\left(F_{\mu \nu}^{j} F^{j \mu \nu}\right)+\sum_{j=1}^{N}\left[\frac{v_{j}^{2}}{2} e^{\frac{2 \sigma_{j}}{v_{j}}}\left(A_{\mu, j}-A_{\mu, j-1}\right)^{2}\right]\right\} .
$$

A ação é invariante de escala só se (4.12) é satisfeita. Até aqui temos estudado uma teoria puramente $4 D$.

Por outro lado vamos estudar a desconstrução de uma teoria $5 D$, considerando a formulação para a métrica de [29] nas teorias $\mathrm{AdS}_{5}$ que inclui o radion explicitamente.

A métrica que contém o campo do radion é dada por

$$
d s^{2}=e^{-2(A+F)} \eta_{\mu \nu} d x^{\mu} d x^{\nu}-(1+2 F)^{2} d y^{2}
$$

onde $A(y)=k y, R=1 / k \mathrm{e}$

$$
\mathbf{F}=\frac{r(x)}{\Lambda_{r}} e^{2 k(y-L)}
$$

é a flutuação correspondente ao efeito do radion. A relação (4.15) é obtida impondo que a métrica (4.14) resolve as equações de einstein, e com a condição que $r(x)$ é um campo escalar $4 D$ canonicamente normalizado. 
Vamos escrever uma Teoria de gauge $5 D$ no caso abeliano ${ }^{3}$

$$
\begin{aligned}
S_{5}^{A} & =\int d^{4} x d y \sqrt{g}\left[-\frac{1}{4 g_{5}^{2}} F_{M N}^{2}\right] \\
& =\int d^{4} x d y \sqrt{g}\left[-\frac{1}{4 g_{5}^{2}} e^{4(k y+\mathbf{F})} F_{\mu \nu} F^{\mu \nu}+\frac{1}{2 g_{5}^{2}} \frac{1}{(1+2 \mathbf{F})^{2}} e^{2(k y+\mathbf{F})}\left(\partial_{5} A_{\mu}-\partial_{\mu} A_{5}\right)^{2}\right],
\end{aligned}
$$

neste caso $\sqrt{g}=e^{-4(A+\mathbf{F})}(1+2 \mathbf{F})$ então que pode ser simplifica a

$$
\begin{aligned}
S_{5}^{A}=\int d^{4} x d y & \frac{1}{g_{5}^{2}}\left[-\frac{1}{4}(1+2 \mathbf{F}) F_{\mu \nu} F^{\mu \nu}\right. \\
+ & \left.\frac{1}{2} \frac{e^{-2(k y+\mathbf{F})}}{(1+2 \mathbf{F})}\left(\partial_{5} A_{\mu}-\partial_{\mu} A_{5}\right)^{2}\right],
\end{aligned}
$$

com $g_{5}$ sendo o acoplamento de gauge $5 D$. Vamos a discretizar essa ação para tentar identificar a presença do dílaton na $T Q H C 4 D$, dado que o radion está presente na teoria contínua.

A ação discretizada é:

$$
\begin{aligned}
S_{5}^{A}=\frac{\ell}{g_{5}^{2}} \int d^{4} x \sum_{j=0}^{N} & {\left[-\frac{1}{4 g_{5}^{2}}\left(1+2 \mathbf{F}_{\mathbf{j}}\right)\left(F_{\mu \nu}^{j} F^{j \mu \nu}\right)\right.} \\
+ & \left.\frac{1}{2} \frac{e^{-2\left(k \ell j+\mathbf{F}_{\mathbf{j}}\right)}}{\left(1+2 \mathbf{F}_{\mathbf{j}}\right)}\left(\partial_{\mu} A_{5}^{j}-\frac{A_{\mu}^{j}-A_{\mu}^{j-1}}{\ell}\right)^{2}\right] .
\end{aligned}
$$

onde $\ell$ é o espaçamento entre os pontos na dimensão extra, uma vez feita a discretização e $\mathbf{F}_{\mathbf{j}}$ é a discretização da flutuação dada por

\footnotetext{
${ }^{3} \mathrm{O}$ caso não Abeliano é semelhante
} 


$$
\mathbf{F}_{\mathbf{j}}=\frac{r(x)}{\Lambda_{r}} e^{2 k \ell(j-N)}
$$

É possível por meio de fixação de gauge escolher $A_{5} \rightarrow 0$. Em primeira ordem em $\mathbf{F}_{\mathbf{j}}$ temos que

$$
\begin{aligned}
S_{5}^{A}=\frac{\ell}{g_{5}^{2}} \int d^{4} x \sum_{j=0}^{N} & {\left[-\frac{1}{4 g_{5}^{2}}\left(1+2 \mathbf{F}_{\mathbf{j}}\right)\left(F_{\mu \nu}^{j} F^{j \mu \nu}\right)\right.} \\
+ & \left.\frac{1}{2}\left(1-4 \mathbf{F}_{\mathbf{j}}\right)\left(\frac{A_{\mu}^{j}-A_{\mu}^{j-1}}{\ell}\right)^{2}\right] .
\end{aligned}
$$

Voltando à expressão (4.13) onde introduzimos os campos $\sigma_{j}$, tínhamos considerado que para cada campo correspondente a $j$ na ação (4.8), sua expressão virava invariante de escala multiplicando por uma potência adequada de $e^{\frac{\sigma_{j}}{v_{j}}}$; com um correspondente $j$.

Podemos estabelecer a equivalência da teoria de gauge $4 D$ (4.13) desconstruída e a teoria de gauge $5 D$ discretizada (4.20) sem o radion, estabelecendo o dicionário entre ambas teorias ver tabela (4.1):

\begin{tabular}{ccc} 
Teoria $4 D$ desconstruída & & Teoria $5 D$ discretizada \\
\hline$v_{j}$ & $\leftrightarrow$ & $\frac{e^{-k \ell j}}{g \ell} \equiv v e^{-k \ell j}$ \\
$\frac{1}{g^{2}}$ & $\leftrightarrow$ & $\frac{\ell}{g_{5}^{2}}$ \\
\hline
\end{tabular}

Tabela 4.1: Dicionário entre a teoria de gauge desconstruída $4 D$ e a teoria de gauge com uma dimensão extra curva discretizada $5 D$ sem o radion.

Agora estabelecemos o dicionário entre as teorias (4.13) e (4.20) com as observações mencionadas incluindo o radion. Vamos considerar de (4.13) até a primeira ordem em $\sigma_{j}$ o dicionário que inclui o radion e $\sigma_{j}$ é mostrado na seguinte tabela 4.2 . 


\begin{tabular}{ccc} 
Teoria $4 D$ que contém $\sigma_{j}$ & & Teoria $5 D$ discretizada com o radion \\
\hline$\sigma_{j}$ & $\leftrightarrow$ & $-2 \frac{v_{j}}{\Lambda_{r}} r(x) e^{2 k \ell(j-N)}$ \\
\hline
\end{tabular}

Tabela 4.2: Dicionário que mostra a equivalência entre $\sigma_{j}$ que faz com que a teoria desconstruída $4 D$ seja invariante de escala e o campo do radion que vem da teoria $5 D$ discretizada.

O fator $e^{k \ell(j-N)}$ é importante só para $j \approx N$. Além disso se $\sigma_{j}$ fosse só para $j=N$ então de acordo com [2] $\sigma_{N}$ é o autentico bóson de Nambu Goldstone segundo o procedimento de Coleman. Então podemos interpretar que o radion é o dílaton da quebra da simetria de escala localizado segundo (4.15) perto da brana IR. Até aqui establecimos a existencia de um dílaton, tal como no caso $\mathrm{AdS}_{5}$. Mas agora temos que ver se é possível dar uma massa para ele de tal forma que ela seja relativamente leve. Faremos isso a seguir.

\subsection{Estabilização entre as escalas UV e IR}

Temos visto que as $T Q H C$ desconstruídas no limite do contínuo, é dizer quando o número de sítios vai para o infinito, resultam em $\mathrm{AdS}_{5}$ junto com o dicionário entre ambas teorias. Nesta seção vamos achar a massa para o radion a partir da discretização de $G W$ do capítulo anterior, equação (3.10), que fez possível estabilizar a longitude da dimensão extra.

Tínhamos considerado um escalar se propagando na bulk com interação nas branas UV e IR. Vamos nos concentrar a partir da obtenção do potencial efetivo antes de fazer a integração em $y$. O potencial efetivo é valor negativo dentro da integral quadridimensional dado por ( ver (3.15)) 


$$
\begin{aligned}
& V=\frac{1}{2} \int_{-L}^{L} d y {\left[A^{2}\left(8 k^{2}+4 k^{2} \sqrt{4+\frac{m^{2}}{k^{2}}}+2 m^{2}\right) e^{2 k|y| \nu}\right.} \\
&\left.B^{2}\left(8 k^{2}-4 k^{2} \sqrt{4+\frac{m^{2}}{k^{2}}}+2 m^{2}\right) e^{-2 k|y| \nu}\right],
\end{aligned}
$$

que pode ser escrito como

$$
\begin{aligned}
V=\int_{0}^{L} d y & {\left[A^{2}\left(2 k^{2}\left(4+\frac{2 m^{2}}{k^{2}}\right)+4 k^{2} \sqrt{4+\frac{m^{2}}{k^{2}}}\right) e^{2 k|y| \nu}\right.} \\
& \left.+B^{2}\left(2 k^{2}\left(4+\frac{2 m^{2}}{k^{2}}\right)-4 k^{2} \sqrt{4+\frac{m^{2}}{k^{2}}}\right) e^{-2 k|y| \nu}\right]
\end{aligned}
$$

Considerando também que $\nu=\sqrt{4+\frac{m^{2}}{k^{2}}}$. A seguir fazemos a discretização em $y$, então $y \rightarrow \ell j$, sendo que $\ell N=L$, então (4.22) fica segundo

$$
V=\sum_{j=0}^{N} \ell\left[A^{2} 2 k^{2} \nu(\nu+2) e^{2 k \ell j \nu}+B^{2} 2 k^{2} \nu(\nu-2) e^{-2 k \ell j \nu}\right]
$$

agora usamos a aproximação $\frac{m}{k} \ll 1 \Longrightarrow \nu=2+\epsilon, \epsilon=\frac{m^{2}}{4 k^{2}}$. Podemos desprezar termos proporcionais a $\epsilon^{2}$ para obter

$$
V=\sum_{j=0}^{N} \ell\left[A^{2} 2 k^{2}(8+6 \epsilon) e^{2 k \ell j(2+\epsilon)}+B^{2} 2 k^{2}(2 \epsilon) e^{-2 k \ell j(2+\epsilon)}\right]
$$

Usando as expressões (3.20), (3.21) para A e B, em termos de $\epsilon$, e considerando 
só até termos proporcionais a $\epsilon$ obtemos

$$
V=\sum_{j=0}^{N} \ell\left\{e^{-8 k L-4 \epsilon k L}\left(v_{v}-v_{h}\right)^{2} 2 k^{2}(8+6 \epsilon) e^{4 k \ell j+2 k \ell j \epsilon}\right\},
$$

onde a soma esta em $j$. É possível obter a soma ${ }^{4}$ :

$$
V=\ell e^{-8 k L-4 \epsilon k L}\left(v_{v}-v_{h}\right)^{2} 16 k^{2}\left[\frac{1-e^{(4 k \ell+2 k \ell \epsilon)(N+1)}}{1-e^{4 k \ell+2 k \ell \epsilon}}\right]
$$

onde justamente vamos usar que $L=\ell N$.

\section{Definimos}

$$
\langle\varphi\rangle=f e^{-k L}
$$

Vamos expressar $V$ em termos de $\langle\varphi\rangle$,

$$
V=\ell\left[v_{v}-v_{h}\left(\frac{\langle\varphi\rangle}{f}\right)^{\epsilon}\right]^{2} 16 k^{2}\left[\frac{\left(\frac{\langle\varphi\rangle}{f}\right)^{8+2 \epsilon}-\left(\frac{\langle\varphi\rangle}{f}\right)^{4} e^{k \ell(4+2 \epsilon)}}{1-e^{k \ell(4+2 \epsilon)}}\right]
$$

Notar que no potencial (4.28) $\langle\varphi\rangle$ depende de $L$ que é o parâmetro livre. A seguir vamos minimizar o potencial $V$ por isso calculamos a primeira derivada do $V$ respeito do $\langle\varphi\rangle$, onde obtemos

\footnotetext{
${ }^{4}$ É uma serie geométrica em potências de $e^{4 k \ell+2 k \ell \epsilon}$
} 


$$
\begin{aligned}
\frac{\partial V}{\partial\langle\varphi\rangle}= & -\frac{32 \ell k^{2} \epsilon\left(\frac{\langle\varphi\rangle}{f}\right)^{\epsilon-1}\left(-e^{4 \ell k+2 \ell k \epsilon}\left(\frac{\langle\varphi\rangle}{f}\right)^{4}+\left(\frac{\langle\varphi\rangle}{f}\right)^{8+2 \epsilon}\right) v_{h}\left(-\left(\frac{\langle\varphi\rangle}{f}\right)^{\epsilon} v_{h}+v_{v}\right)}{\left(1-e^{4 a k+2 a k \epsilon}\right) f} \\
& +\frac{16 \ell k^{2}\left(-4 e^{4 \ell k+2 \ell k \epsilon}\left(\frac{\langle\varphi\rangle}{f}\right)^{3}+(8+2 \epsilon)\left(\frac{\langle\varphi\rangle}{f}\right)^{7+2 \epsilon}\right)\left(-\left(\frac{\langle\varphi\rangle}{f}\right)^{\epsilon} v_{h}+v_{v}\right)^{2}}{f\left(1-e^{4 \ell k+2 a k \epsilon}\right)} .
\end{aligned}
$$

Resolvendo $\frac{\partial V}{\partial\langle\varphi\rangle}=0$, e o potencial $V$ é mínimo quando se satisfaz

$$
\left(\frac{\langle\varphi\rangle}{f}\right)^{\epsilon}=\frac{v_{v}}{v_{h}},
$$

que é o mesmo resultado obtido no capítulo anterior (3.26). Agora vamos obter uma massa efetiva para o qual achamos a segunda derivada de $V$ respeito de $\partial\langle\varphi\rangle$

$$
\begin{aligned}
\frac{\partial^{2} V}{\partial\langle\varphi\rangle^{2}}= & \frac{32 \ell k^{2} \epsilon^{2}\left(\frac{\phi}{f}\right)^{-2+2 \epsilon}\left(-\frac{e^{4 \ell k+2 \ell \ell \epsilon} \phi^{4}}{f^{4}}+\left(\frac{\phi}{f}\right)^{8+2 \epsilon}\right) v_{h}^{2}}{\left(1-e^{4 \ell k+2 \ell k \epsilon}\right) f^{2}} \\
& -\frac{64 \ell k^{2} \epsilon\left(\frac{\phi}{f}\right)^{-1+\epsilon}\left(-\frac{4 e^{4 \ell k+2 \ell \ell \epsilon} \phi^{3}}{f^{4}}+\frac{(8+2 \epsilon)\left(\frac{\phi}{f}\right)^{7+2 \epsilon}}{f}\right) v_{h}\left(-\left(\frac{\phi}{f}\right)^{\epsilon} v_{h}+v_{v}\right)}{\left(1-e^{4 \ell k+2 \ell k \epsilon}\right) f} \\
& -\frac{32 \ell k^{2}(-1+\epsilon) \epsilon\left(\frac{\phi}{f}\right)^{-2+\epsilon}\left(-\frac{e^{4 \ell k+2 \ell k \epsilon} \phi^{4}}{f^{4}}+\left(\frac{\phi}{f}\right)^{8+2 \epsilon}\right) v_{h}\left(-\left(\frac{\phi}{f}\right)^{\epsilon} v_{h}+v_{v}\right)}{\left(1-e^{4 \ell k+2 \ell k \epsilon}\right) f^{2}} \\
& +\frac{16 \ell k^{2}\left(-\frac{12 e^{4 \ell k+2 \ell k \epsilon} \phi^{2}}{f^{4}}+\frac{(7+2 \epsilon)(8+2 \epsilon)\left(\frac{\phi}{f}\right)^{6+2 \epsilon}}{f^{2}}\right)\left(-\left(\frac{\phi}{f}\right)^{\epsilon} v_{h}+v_{v}\right)^{2}}{1-e^{4 \ell k+2 \ell k \epsilon}} .
\end{aligned}
$$


Avaliamos no valor obtido em (4.30) para obter a massa para $\varphi$ dada por

$$
m_{\varphi}^{2}=\left.\frac{\partial^{2} V}{\partial \varphi^{2}}\right|_{\varphi=f\left(\frac{v_{v}}{v_{h}}\right)^{1 / \epsilon}}=\frac{32 \ell v_{v}^{2} k^{2} \epsilon^{2}}{f^{2}\left(1-e^{4 k \ell+2 k \ell \epsilon}\right)}\left[-e^{-2 k L} e^{4 k \ell+2 k \ell \epsilon}+e^{-6 k L} e^{-2 k L \epsilon}\right]
$$

substituindo o valor de $f=\sqrt{\frac{24 M^{3}}{k}}$, teremos imediatamente

$$
m_{\varphi}^{2}=\frac{4 k \ell v_{v}^{2} k^{2} \epsilon^{2}}{3 M^{3}\left(1-e^{4 k \ell+2 k \ell \epsilon}\right)}\left[-e^{-2 k L} e^{4 k \ell+2 k \ell \epsilon}+e^{-6 k L} e^{-2 k L \epsilon}\right]
$$

analogamente ao mecanismo de GW. Neste caso estamos trabalhando na teoria puramente quadridimensional.

Então temos obtido a massa do radion na teoria $4 D$, vemos que depende do parâmetro do espaçamento de rede $\ell$, além disso tem uma supressão devido ao parâmetro muito pequeno $\epsilon$, o fator exponencial nos diz que o dílaton está localizado perto do sítio onde a escala é menor (IR).

Recuperamos o contínuo tomando $N \rightarrow \infty$ o que é o mesmo quando $\ell \rightarrow o$

$$
\lim _{N \rightarrow \infty} m_{\varphi}^{2}=\frac{2 k^{2} v_{v}^{2} \epsilon^{2}}{3 M^{3}(2+\epsilon)} e^{-2 k L(3+\epsilon)}\left[-1+e^{2 k L(2+\epsilon)}\right]
$$

Junto com o resultado do capítulo anterior que $k L \approx 36$, e que $\epsilon=\frac{m^{2}}{4 k^{2}}$, $m / k \ll 1$, obtemos

$$
\lim _{N \rightarrow \infty} m_{\varphi}^{2}=\frac{k^{2} v_{v}^{2} \epsilon^{2}}{3 M^{3}} e^{-2 k L}
$$

Então no limite do contínuo recuperamos o valor da massa obtida segundo o mecanismo de $G W$. Esse procedimento mostra que, a principio, as TQHC podem conter um dílaton leve (em relação as massas dos modos de excitados do diagrama de quiver ).

É interessante estudar outras formas de dar massas aos dílatons nas TQHC. 
Dado que essas teorias tem uma grande hierarquia de escalas, elas devem apresentar um comportamento efetivamente quase-conforme, mesmo para $N$ finito. É de esperasse que exista um dílaton associado com esse comportamento.

Mas o mecanismo de quebra explícita responsável pelo potencial do dílaton pode ser bem diferente do apresentado aqui. Uma possibilidade é que o potencial é radiativamente gerado a baixas energias. Essa possibilidade será estudada no futuro. 


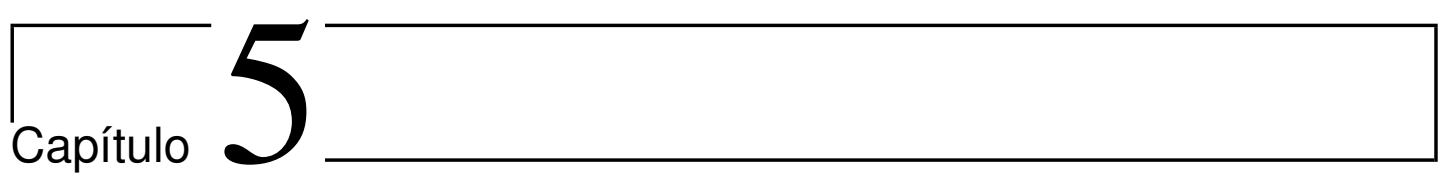

\section{Conclusão}

O Modelo Padrão $(M P)$ continua sendo uma teoria extremadamente bem sucedida, pois explica os dados experimentais obtidos no regime de energias exploradas até hoje. Porém, o MP torna-se instável no regime de energias onde os efeitos da gravidade são importantes. Este modelo apresenta outros problemas além dos mencionados na Introdução, entre eles, o problema da hierarquia de gauge e o problema da hierarquia das massas dos férmions, o qual nos motiva às procuras de nova física além do MP. Por isso, estudamos algumas teorias onde é possível explicar dinamicamente essas hierarquias. Nesse sentido estudamos teorias com uma dimensão extra curva, onde a compactificação é feita no obifold $S_{1} / Z_{2}$, utilizando a métrica de Randall-Sundrum. Este tipo de teorias, conhecidas como Warped Extra Dimensions, pertencem às teorias do tipo $\mathrm{AdS}_{5}$. O resultado imediato foi explicar a grande diferença entre as escalas de energias de Planck e TeV. Também vimos que ao permitir aos férmions a liberdade de se propagarem na bulk, obtemos que os modos zero estão localizados nas branas (UV, IR). Isto é, quando identificamos o parâmetro $c$ nas funções de onda 5-dimensionais, observamos que alguns férmions têm maior acoplamento com o Higgs do que outros, o 
que gera dinamicamente a hierarquia das massas dos férmions. Não obstante, há um problema de ter que trabalhar com teorias 5-dimensionais, estas teorias são não renormalizáveis.

Nesta dissertação observamos que as Teorias Quiver de Hierarquia Completa $(T Q H C)$, que são puramente teorias 4-dimensionais $(4 D)$, possuem as vantagens das teorias $\mathrm{AdS}_{5}$, já que elas geram grandes hierarquias, o que é aproveitado para resolver os problemas de hierarquia do Modelo Padrão. Além disso temos observado que as $T Q H C$ no limite de fazer o número de sítios tender ao infinito obtemos as teorias $\mathrm{AdS}_{5}$.

Apresentamos no Capítulo 3 que ao trabalhar em Teorias $\mathrm{AdS}_{5}$, a dimensão extra possui um raio de compactificação que torna essas teorias gravitacionalmente estáveis. Com o objetivo de estabilizar a longitude da dimensão extra introduzimos o mecanismo Goldberger-Wise $(G W)$, o qual permitiu identificar a massa de um escalar, conhecido como radion, cuja massa é essencialmente menor do que os modos de excitação de Kaluza Klein das outras partículas. Por outro lado encontramos que o radion tem localização perto da brana IR.

Também estudamos alguns aspetos das $T Q H C$ relacionados com a quebra da simetria de escala. Vimos que é possível ter um dílaton leve nessas teorias $4 D$. Por meio de um potencial semelhante ao mecanismo $G W$.

Como uma perspetiva futura, esperamos estudar outras formas de gerar um potencial para o dílaton. Específicamente, gerar o potencial para o dílaton radiativamente, a baixas energias. Além disso fica a questão se a massa do dílaton permanecerá sendo logo de ter feito as correções radiativas. 
Apêndices 



\section{APÊNDICE A}

Neste apêndice mostramos os símbolos de Christoffel não nulos para a métrica de Randall-Sundrum (2.3). Para a obtenção da conexão de spin do capítulo dois.

Os símbolos de Christoffel são dados por

as letras latinas são usadas para os índices 5-dimensionais $(0,1,2,3,5)$ de onde

$$
\begin{gathered}
\Gamma_{11}^{5}=k e^{-2 k y}, \Gamma_{15}^{1}=\Gamma_{51}^{1}=-k, \\
\Gamma_{22}^{5}=k e^{-2 k y}, \Gamma_{25}^{2}=\Gamma_{52}^{2}=-k, \\
\Gamma_{33}^{5}=k e^{-2 k y}, \Gamma_{35}^{3}=\Gamma_{53}^{3}=-k, \\
\Gamma_{00}^{5}=-k e^{-2 k y}, \Gamma_{05}^{0}=\Gamma_{50}^{0}=-k .
\end{gathered}
$$




\section{APÊNDICE B}

Neste apêndice vamos mostrar que a partir da ação de Hilbert-Einstein 5-dimensional com métrica dada por (3.1) obtemos uma ação efetiva puramente quadridimensional, vamos começar mostrando o cálculo da curvatura quadridimensional com a métrica dada por $g \mu \nu(x)$,considerando a notação para as derivadas da métrica quadridimensional com a convenção para índices mencionado no capítulo 2 ,

$$
\partial_{\rho} g_{\mu \nu} \equiv g_{\mu \nu x^{\rho}},
$$


por tanto $R_{4}$ é dado por

$$
\begin{aligned}
& R_{4}=\frac{g_{22 x^{3}} g_{33} x^{3}}{2 g_{22} g_{33}{ }^{2}}+\frac{g_{11 x^{3}} g_{33 x^{3}}}{2 g_{11} g_{33}{ }^{2}}+\frac{g_{00 x^{3}} g_{33 x^{3}}}{2 g_{00} g_{33}{ }^{2}}-\frac{g_{33 x^{2} x^{2}}}{g_{22} g_{33}} \\
& +\frac{\left(g_{33 x^{2}}\right)^{2}}{2 g_{22} g_{33^{2}}{ }^{2}}+\frac{g_{22 x^{2}} g_{33 x^{2}}}{2 g_{22}{ }^{2} g_{33}}-\frac{g_{11 x^{2}} g_{33} x^{2}}{2 g_{11} g_{22} g_{33}}-\frac{g_{00 x^{2}} g_{33} x^{2}}{2 g_{00} g_{22} g_{33}} \\
& -\frac{g_{33 x^{1} x^{1}}}{g_{11} g_{33}}+\frac{\left(g_{33 x^{1}}\right)^{2}}{2 g_{11} g_{33^{2}}}-\frac{g_{22 x^{1}} g_{33 x^{1}}}{2 g_{11} g_{22} g_{33}}+\frac{g_{11 x^{1}} g_{33 x^{1}}}{2 g_{11^{2}} g_{33}} \\
& -\frac{g_{00 x^{1}} g_{33} x^{1}}{2 g_{00} g_{11} g_{33}}-\frac{g_{33 x^{0} x^{0}}}{g_{00} g_{33}}+\frac{\left(g_{33 x^{0}}\right)^{2}}{2 g_{00} g_{33}{ }^{2}}-\frac{g_{22 x^{0}} g_{33} x^{0}}{2 g_{00} g_{22} g_{33}} \\
& -\frac{g_{11 x^{0}} g_{33 x^{0}}}{2 g_{00} g_{11} g_{33}}+\frac{g_{00 x^{0}} g_{33 x^{0}}}{2 g_{00}{ }^{2} g_{33}}-\frac{g_{22 x^{3} x^{3}}}{g_{22} g_{33}}+\frac{\left(g_{22 x^{3}}\right)^{2}}{2 g_{22^{2} g_{33}}} \\
& -\frac{g_{11 x^{3}} g_{22 x^{3}}}{2 g_{11} g_{22} g_{33}}-\frac{g_{00 x^{3}} g_{22 x^{3}}}{2 g_{00} g_{22} g_{33}}-\frac{g_{11 x^{3} x^{3}}}{g_{11} g_{33}}+\frac{\left(g_{11} x^{3}\right)^{2}}{2 g_{11}{ }^{2} g_{33}} \\
& -\frac{g_{00 x^{3}} g_{11 x^{3}}}{2 g_{00} g_{11} g_{33}}-\frac{g_{00 x^{3} x^{3}}}{g_{00} g_{33}}+\frac{\left(g_{00 x^{3}}\right)^{2}}{2 g_{00} g_{33}}+\frac{g_{11 x^{2}} g_{22 x^{2}}}{2 g_{11} g_{22}{ }^{2}} \\
& +\frac{g_{00 x^{2}} g_{22 x^{2}}}{2 g_{00} g_{22^{2}}}-\frac{g_{22 x^{1} x^{1}}}{g_{11} g_{22}}+\frac{\left(g_{22 x^{1}}\right)^{2}}{2 g_{11} g_{22}{ }^{2}}+\frac{g_{11 x^{1}} g_{22 x^{1}}}{2 g_{11^{2}} g_{22}} \\
& -\frac{g_{00 x^{1}} g_{22 x^{1}}}{2 g_{00} g_{11} g_{22}}-\frac{g_{22 x^{0} x^{0}}}{g_{00} g_{22}}+\frac{\left(g_{22 x^{0}}\right)^{2}}{2 g_{00} g_{22^{2}}}-\frac{g_{11 x^{0}} g_{22 x^{0}}}{2 g_{00} g_{11} g_{22}} \\
& +\frac{g_{00 x^{0}} g_{22 x^{0}}}{2 g_{00}{ }^{2} g_{22}}-\frac{g_{11 x^{2} x^{2}}}{g_{11} g_{22}}+\frac{\left(g_{11 x^{2}}\right)^{2}}{2 g_{11}^{2} g_{22}}-\frac{g_{00 x^{2}} g_{11} x^{2}}{2 g_{00} g_{11} g_{22}} \\
& -\frac{g_{00 x^{2} x^{2}}}{g_{00} g_{22}}+\frac{\left(g_{00 x^{2}}\right)^{2}}{2 g_{00}{ }^{2} g_{22}}+\frac{g_{00 x^{1}} g_{11 x^{1}}}{2 g_{00} g_{11}{ }^{2}}-\frac{g_{11 x^{0} x^{0}}}{g_{00} g_{11}} \\
& +\frac{\left(g_{11 x^{0}}\right)^{2}}{2 g_{00} g_{11}^{2}}+\frac{g_{00 x^{0}} g_{11 x^{0}}}{2 g_{00}{ }^{2} g_{11}}-\frac{g_{00 x^{1} x^{1}}}{g_{00} g_{11}}+\frac{\left(g_{00 x^{1}}\right)^{2}}{2 g_{00}{ }^{2} g_{11}} \text {, }
\end{aligned}
$$

agora se mostramos o escalar de Ricci 5-dimensional calculado usando a métrica (3.1) 


$$
\begin{aligned}
& R_{5}=-\frac{2 e^{\frac{2 k|y| T}{r_{c}}} T_{x^{3} x^{3}}}{g_{33} T}+\frac{6 k|y| e^{\frac{2 k|y| T}{r_{c}}} T_{x^{3}} x^{3}}{g_{33} r_{c}}+\frac{4 k|y| e^{\frac{2 k|y| T}{r_{c}}}\left(T_{x^{3}}\right)^{2}}{g_{33} r_{c} T} \\
& -\frac{6 k^{2}|y|^{2} e^{\frac{2 k|y| T}{r_{c}}}\left(T_{x^{3}}\right)^{2}}{g_{33} r_{c}^{2}}+\frac{g_{33 x^{3}} e^{\frac{2 k|y| T}{r_{c}}} T_{x^{3}}}{g_{33^{2}} T}-\frac{g_{22 x^{3}} e^{\frac{2 k|y| T}{r_{c}}} T_{x^{3}}}{g_{22} g_{33} T} \\
& -\frac{g_{11 x^{3}} e^{\frac{2 k|y| T}{r_{c}}} T_{x^{3}}}{g_{11} g_{33} T}-\frac{g_{00 x^{3}} e^{\frac{2 k|y| T}{r_{c}}} T_{x^{3}}}{g_{00} g_{33} T}-\frac{3 g_{33 x^{3}} k|y| e^{\frac{2 k|y| T}{r_{c}}} T_{x^{3}}}{g_{33^{2}} r_{c}} \\
& +\frac{3 g_{22 x^{3}} k|y| e^{\frac{2 k|y| T}{r_{c}}} T_{x^{3}}}{g_{22} g_{33} r_{c}}+\frac{3 g_{11 x^{3}} k|y| e^{\frac{2 k|y| T}{r_{c}}} T_{x^{3}}}{g_{11} g_{33} r_{c}}+\frac{3 g_{00 x^{3}} k|y| e^{\frac{2 k|y| T}{r_{c}}} T_{x^{3}}}{g_{00} g_{33} r_{c}} \\
& -\frac{2 e^{\frac{2 k|y| T}{r_{c}}} T_{x^{2} x^{2}}}{g_{22} T}+\frac{6 k|y| e^{\frac{2 k|y| T}{r_{c}}} T_{x^{2} x^{2}}}{g_{22} r_{c}}+\frac{4 k|y| e^{\frac{2 k|y| T}{r_{c}}}\left(T_{x^{2}}\right)^{2}}{g_{22} r T} \\
& -\frac{6 k^{2}|y|^{2} e^{\frac{2 k|y| T}{r_{c}}}\left(T_{x^{2}}\right)^{2}}{g_{22} r_{c}^{2}}-\frac{g_{33 x^{2}} e^{\frac{2 k|y| T}{r_{c}}} T_{x^{2}}}{g_{22} g_{33} T}+\frac{g_{22 x^{2}} e^{\frac{2 k|y| T}{r_{c}}} T_{x^{2}}}{g_{22^{2}}{ }^{2}} \\
& -\frac{g_{11 x^{2}} e^{\frac{2 k|y| T}{r_{c}}} T_{x^{2}}}{g_{11} g_{22} T}-\frac{g_{00 x^{2}} e^{\frac{2 k|y| T}{r_{c}}} T_{x^{2}}}{g_{00} g_{22} T}+\frac{3 g_{33 x^{2}} k|y| e^{\frac{2 k|y| T}{r_{c}}} T_{x^{2}}}{g_{22} g_{33} r_{c}} \\
& -\frac{3 g_{22 x^{2}} k|y| e^{\frac{2 k|y| T}{r_{c}}} T_{x^{2}}}{g_{22^{2}} r_{c}}+\frac{3 g_{11 x^{2}} k|y| e^{\frac{2 k|y| T}{r_{c}}} T_{x^{2}}}{g_{11} g_{22} r_{c}}+\frac{3 g_{00 x^{2}} k|y| e^{\frac{2 k|y| T}{r_{c}}} T_{x^{2}}}{g_{00} g_{22} r_{c}} \\
& -\frac{2 e^{\frac{2 k|y| T}{r_{c}}} T_{x^{1} x^{1}}}{g_{11} T}+\frac{6 k|y| e^{\frac{2 k|y| T}{r_{c}}} T_{x^{1} x^{1}}}{g_{11} r_{c}}+\frac{4 k|y| e^{\frac{2 k|y| T}{r_{c}}}\left(T_{x^{1}}\right)^{2}}{g_{11} r_{c} T} \\
& -\frac{6 k^{2}|y|^{2} e^{\frac{2 k|y| T}{r_{c}}}\left(T_{x^{1}}\right)^{2}}{g_{11} r_{c}^{2}}-\frac{g_{33 x^{1}} e^{\frac{2 k|y| T}{r_{c}}} T_{x^{1}}}{g_{11} g_{33} T}-\frac{g_{22 x^{1}} e^{\frac{2 k|y| T}{r_{c}}} T_{x^{1}}}{g_{11} g_{22} T} \\
& +\frac{g_{11 x^{1}} e^{\frac{2 k|y| T}{r_{c}}} T_{x^{1}}}{g_{11^{2}} T}-\frac{g_{00 x^{1}} e^{\frac{2 k|y| T}{r_{c}}} T_{x^{1}}}{g_{00} g_{11} T}+\frac{3 g_{33_{x^{1}}} k|y| e^{\frac{2 k|y| T}{r_{c}}} T_{x^{1}}}{g_{11} g_{33} r_{c}} \\
& +\frac{3 g_{22_{x^{1}}} k|y| e^{\frac{2 k|y| T}{r_{c}}} T_{x^{1}}}{g_{11} g_{22} r_{c}}-\frac{3 g_{11 x^{1}} k|y| e^{\frac{2 k|y| T}{r_{c}}} T_{x^{1}}}{g_{11^{2}} r_{c}}+\frac{3 g_{00 x^{1}} k|y| e^{\frac{2 k|y| T}{r_{c}}} T_{x^{1}}}{g_{00} g_{11} r_{c}} \\
& -\frac{2 e^{\frac{2 k|y| T}{r_{c}}} T_{x^{0} x^{0}}}{g_{00} T}+\frac{6 k|y| e^{\frac{2 k|y| T}{r_{c}}} T_{x^{0} x^{0}}}{g_{00} r_{c}}+\frac{4 k|y| e^{\frac{2 k|y| T}{r_{c}}}\left(T_{x^{0}}\right)^{2}}{g_{00} r_{c} T} \\
& -\frac{6 k^{2}|y|^{2} e^{\frac{2 k|y| T}{r_{c}}}\left(T_{x^{0}}\right)^{2}}{g_{00} r_{c}^{2}}-\frac{g_{33 x^{0}} e^{\frac{2 k|y| T}{r_{c}}} T_{x^{0}}}{g_{00} g_{33} T}-\frac{g_{22 x^{0}} e^{\frac{2 k|y| T}{r_{c}}} T_{x^{0}}}{g_{00} g_{22} T} \\
& -\frac{g_{11 x^{0}} e^{\frac{2 k|y| T}{r_{c}}} T_{x^{0}}}{g_{00} g_{11} T}+\frac{g_{00 x^{0}} e^{\frac{2 k|y| T}{r}} T_{x^{0}}}{g_{00^{2}} T}+\frac{3 g_{33 x^{0}} k|y| e^{\frac{2 k|y| T}{r_{c}}} T_{x^{0}}}{g_{00} g_{33} r_{c}} \\
& +\frac{3 g_{22_{x^{0}}} k|y| e^{\frac{2 k|y| T}{r_{c}}} T_{x^{0}}}{g_{00} g_{22} r_{c}}+\frac{3 g_{11 x^{0}} k|y| e^{\frac{2 k|y| T}{r_{c}}} T_{x^{0}}}{g_{00} g_{11} r_{c}}-\frac{3 g_{00 x^{0}} k|y| e^{\frac{2 k|y| T}{r_{c}}} T_{x^{0}}}{g_{00}{ }^{2} r_{c}}
\end{aligned}
$$




$$
\begin{aligned}
& +\frac{g_{22 x^{3}} g_{33 x^{3}} e^{\frac{2 k|y| T}{r_{c}}}}{2 g_{22} g_{33}{ }^{2}}+\frac{g_{11 x^{3}} g_{33 x^{3}} e^{\frac{2 k|y| T}{r_{c}}}}{2 g_{11} g_{33}{ }^{2}}+\frac{g_{00 x^{3}} g_{33 x^{3}} e^{\frac{2 k|y| T}{r_{c}}}}{2 g_{00} g_{33}{ }^{2}}
\end{aligned}
$$

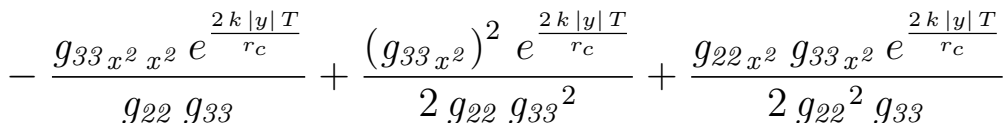

$$
\begin{aligned}
& -\frac{g_{11 x^{2}} g_{33 x^{2}} e^{\frac{2 k|y| T}{r_{c}}}}{2 g_{11} g_{22} g_{33}}-\frac{g_{00 x^{2}} g_{33 x^{2}} e^{\frac{2 k|y| T}{r_{c}}}}{2 g_{00} g_{22} g_{33}}-\frac{g_{33 x^{1} x^{1}} e^{\frac{2 k|y| T}{r_{c}}}}{g_{11} g_{33}} \\
& +\frac{\left(g_{33 x^{1}}\right)^{2} e^{\frac{2 k|y| T}{r_{c}}}}{2 g_{11} g_{33^{2}}}-\frac{g_{22 x^{1}} g_{33 x^{1}} e^{\frac{2 k|y| T}{r_{c}}}}{2 g_{11} g_{22} g_{33}}+\frac{g_{1 x_{x^{1}}} g_{33 x^{1}} e^{\frac{2 k|y| T}{r_{c}}}}{2 g_{11^{2}} g_{33}} \\
& -\frac{g_{00 x^{1}} g_{33 x^{1}} e^{\frac{2 k|y| T}{r_{c}}}}{2 g_{00} g_{11} g_{33}}-\frac{g_{33 x^{0} x^{0}} e^{\frac{2 k|y| T}{r_{c}}}}{g_{00} g_{33}}+\frac{\left(g_{33 x^{0}}\right)^{2} e^{\frac{2 k|y| T}{r_{c}}}}{2 g_{00} g_{33}{ }^{2}} \\
& -\frac{g_{22 x^{0}} g_{33 x^{0}} e^{\frac{2 k|y| T}{r_{c}}}}{2 g_{00} g_{22} g_{33}}-\frac{g_{11 x^{0}} g_{33 x^{0}} e^{\frac{2 k|y| T}{r_{c}}}}{2 g_{00} g_{11} g_{33}}+\frac{g_{00 x^{0}} g_{33 x^{0}} e^{\frac{2 k|y| T}{r_{c}}}}{2 g_{00}{ }^{2} g_{33}} \\
& -\frac{g_{22 x^{3} x^{3}} e^{\frac{2 k|y| T}{r_{c}}}}{g_{22} g_{33}}+\frac{\left(g_{22 x^{3}}\right)^{2} e^{\frac{2 k|y| T}{r_{c}}}}{2 g_{22}{ }^{2} g_{33}}-\frac{g_{11 x^{3}} g_{22 x^{3}} e^{\frac{2 k|y| T}{r_{c}}}}{2 g_{11} g_{22} g_{33}}
\end{aligned}
$$

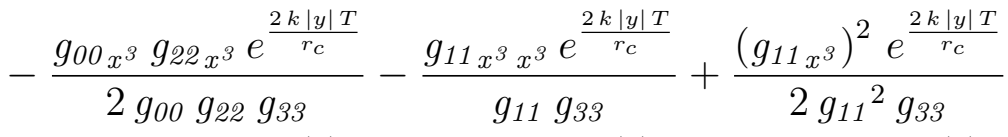

$$
\begin{aligned}
& -\frac{g_{00 x^{3}} g_{11 x^{3}} e^{\frac{2 k|y| T}{r_{c}}}}{2 g_{00} g_{11} g_{33}}-\frac{g_{00 x^{3} x^{3}} e^{\frac{2 k|y| T}{r_{c}}}}{g_{00} g_{33}}+\frac{\left(g_{00 x^{3}}\right)^{2} e^{\frac{2 k|y| T}{r_{c}}}}{2 g_{00}{ }^{2} g_{33}}
\end{aligned}
$$

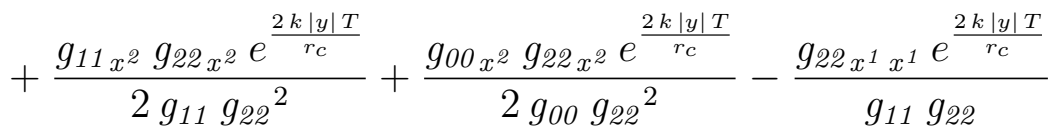

$$
\begin{aligned}
& +\frac{\left(g_{22 x^{1}}\right)^{2} e^{\frac{2 k|y| T}{r_{c}}}}{2 g_{11} g_{22^{2}}{ }^{2}}+\frac{g_{11 x^{1}} g_{22 x^{1}} e^{\frac{2 k|y| T}{r_{c}}}}{2 g_{11^{2} g_{22}}}-\frac{g_{00 x^{1}} g_{22 x^{1}} e^{\frac{2 k|y| T}{r_{c}}}}{2 g_{00} g_{11} g_{22}} \\
& -\frac{g_{22 x^{0} x^{0}} e^{\frac{2 k|y| T}{r_{c}}}}{g_{00} g_{22}}+\frac{\left(g_{22 x^{0}}\right)^{2} e^{\frac{2 k|y| T}{r_{c}}}}{2 g_{00} g_{22}{ }^{2}}-\frac{g_{11 x^{0}} g_{22 x^{0}} e^{\frac{2 k|y| T}{r_{c}}}}{2 g_{00} g_{11} g_{22}}
\end{aligned}
$$

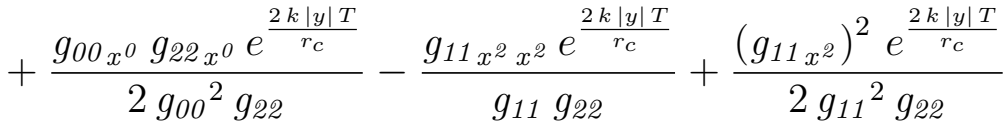

$$
\begin{aligned}
& -\frac{g_{00 x^{2}} g_{1 x^{2}} e^{\frac{2 k|y| T}{r_{c}}}}{2 g_{00} g_{11} g_{22}}-\frac{g_{00 x^{2} x^{2}} e^{\frac{2 k|y| T}{r_{c}}}}{g_{00} g_{22}}+\frac{\left(g_{00 x^{2}}\right)^{2} e^{\frac{2 k|y| T}{r_{c}}}}{2 g_{00^{2}} g_{22}} \\
& +\frac{g_{00 x^{1}} g_{11 x^{1}} e^{\frac{2 k|y| T}{r_{c}}}}{2 g_{00} g_{11}^{2}}-\frac{g_{11 x^{0} x^{0}} e^{\frac{2 k|y| T}{r_{c}}}}{g_{00} g_{11}}+\frac{\left(g_{11 x^{0}}\right)^{2} e^{\frac{2 k|y| T}{r_{c}}}}{2 g_{00} g_{11}{ }^{2}} \\
& +\frac{g_{00 x^{0}} g_{11 x^{0}} e^{\frac{2 k|y| T}{r_{c}}}}{2 g_{00^{2}} g_{11}}-\frac{g_{00_{x^{1}} x^{1}} e^{\frac{2 k|y| T}{r_{c}}}}{g_{00} g_{11}}+\frac{\left(g_{00 x^{1}}\right)^{2} e^{\frac{2 k|y| T}{r_{c}}}}{2 g_{00^{2}} g_{11}}+20 k^{2},
\end{aligned}
$$


depois de identificar em (4) termos cuja soma é proporcional a $R_{4}$, então $R_{5}$ pode-se escrever na forma seguinte

$$
\begin{aligned}
& R_{5}=\frac{2 e^{\frac{2 k|y| T}{r_{c}}} T_{x^{3} x^{3}}}{g_{33} T}+\frac{6 k|y| e^{\frac{2 k|y| T}{r_{c}}} T_{x^{3}} x^{3}}{g_{33} r_{c}}+\frac{4 k|y| e^{\frac{2 k|y| T}{r_{c}}}\left(T_{x^{3}}\right)^{2}}{g_{33} r_{c} T} \\
& -\frac{6 k^{2}|y|^{2} e^{\frac{2 k|y| T}{r_{c}}}\left(T_{x^{3}}\right)^{2}}{g_{33} r_{c}^{2}}+\frac{g_{33} x^{3} e^{\frac{2 k|y| T}{r_{c}}} T_{x^{3}}}{g_{33^{2}} T}-\frac{g_{22 x^{3}} e^{\frac{2 k|y| T}{r_{c}}} T_{x^{3}}}{g_{22} g_{33} T} \\
& -\frac{g_{11 x^{3}} e^{\frac{2 k|y| T}{r_{c}}} T_{x^{3}}}{g_{11} g_{33} T}-\frac{g_{00 x^{3}} e^{\frac{2 k|y| T}{r_{c}}} T_{x^{3}}}{g_{00} g_{33} T}-\frac{3 g_{33 x^{3}} k|y| e^{\frac{2 k|y| T}{r_{c}}} T_{x^{3}}}{g_{33^{2}} r_{c}} \\
& +\frac{3 g_{22 x^{3}} k|y| e^{\frac{2 k|y| T}{r_{c}}} T_{x^{3}}}{g_{22} g_{33} r_{c}}+\frac{3 g_{11 x^{3}} k|y| e^{\frac{2 k|y| T}{r_{c}}} T_{x^{3}}}{g_{11} g_{33} r_{c}}+\frac{3 g_{00 x^{3}} k|y| e^{\frac{2 k|y| T}{r_{c}}} T_{x^{3}}}{g_{00} g_{33} r_{c}} \\
& -\frac{2 e^{\frac{2 k|y| T}{r_{c}}} T_{x^{2} x^{2}}}{g_{22} T}+\frac{6 k|y| e^{\frac{2 k|y| T}{r_{c}}} T_{x^{2} x^{2}}}{g_{22} r_{c}}+\frac{4 k|y| e^{\frac{2 k|y| T}{r_{c}}}\left(T_{x^{2}}\right)^{2}}{g_{22} r T} \\
& -\frac{6 k^{2}|y|^{2} e^{\frac{2 k|y| T}{r_{c}}}\left(T_{x^{2}}\right)^{2}}{g_{22} r_{c}^{2}}-\frac{g_{33 x^{2}} e^{\frac{2 k|y| T}{r_{c}}} T_{x^{2}}}{g_{22} g_{33} T}+\frac{g_{22 x^{2}} e^{\frac{2 k|y| T}{r_{c}}} T_{x^{2}}}{g_{22^{2}}{ }^{2}} \\
& -\frac{g_{11 x^{2}} e^{\frac{2 k|y| T}{r_{c}}} T_{x^{2}}}{g_{11} g_{22} T}-\frac{g_{00 x^{2}} e^{\frac{2 k|y| T}{r_{c}}} T_{x^{2}}}{g_{00} g_{22} T}+\frac{3 g_{33 x^{2}} k|y| e^{\frac{2 k|y| T}{r_{c}}} T_{x^{2}}}{g_{22} g_{33} r_{c}} \\
& -\frac{3 g_{22 x^{2}} k|y| e^{\frac{2 k|y| T}{r_{c}}} T_{x^{2}}}{g_{22^{2}} r_{c}}+\frac{3 g_{11 x^{2}} k|y| e^{\frac{2 k|y| T}{r_{c}}} T_{x^{2}}}{g_{11} g_{22} r_{c}}+\frac{3 g_{00 x^{2}} k|y| e^{\frac{2 k|y| T}{r_{c}}} T_{x^{2}}}{g_{00} g_{22} r_{c}} \\
& -\frac{2 e^{\frac{2 k|y| T}{r_{c}}} T_{x^{1} x^{1}}}{g_{11} T}+\frac{6 k|y| e^{\frac{2 k|y| T}{r_{c}}} T_{x^{1} x^{1}}}{g_{11} r_{c}}+\frac{4 k|y| e^{\frac{2 k|y| T}{r_{c}}}\left(T_{x^{1}}\right)^{2}}{g_{11} r_{c} T} \\
& -\frac{6 k^{2}|y|^{2} e^{\frac{2 k|y| T}{r_{c}}}\left(T_{x^{1}}\right)^{2}}{g_{11} r_{c}^{2}}-\frac{g_{33 x^{1}} e^{\frac{2 k|y| T}{r_{c}}} T_{x^{1}}}{g_{11} g_{33} T}-\frac{g_{22 x^{1}} e^{\frac{2 k|y| T}{r_{c}}} T_{x^{1}}}{g_{11} g_{22} T} \\
& +\frac{g_{11 x^{1}} e^{\frac{2 k|y| T}{r_{c}}} T_{x^{1}}}{g_{11^{2} T} T}-\frac{g_{00 x^{1}} e^{\frac{2 k|y| T}{r_{c}}} T_{x^{1}}}{g_{00} g_{11} T}+\frac{3 g_{33 x^{1}} k|y| e^{\frac{2 k|y| T}{r_{c}}} T_{x^{1}}}{g_{11} g_{33} r_{c}} \\
& +\frac{3 g_{22 x^{1}} k|y| e^{\frac{2 k|y| T}{r_{c}}} T_{x^{1}}}{g_{11} g_{22} r_{c}}-\frac{3 g_{11 x^{1}} k|y| e^{\frac{2 k|y| T}{r_{c}}} T_{x^{1}}}{g_{11}{ }^{2} r_{c}}+\frac{3 g_{00 x^{1}} k|y| e^{\frac{2 k|y| T}{r_{c}}} T_{x^{1}}}{g_{00} g_{11} r_{c}} \\
& -\frac{2 e^{\frac{2 k|y| T}{r_{c}}} T_{x^{0} x^{0}}}{g_{00} T}+\frac{6 k|y| e^{\frac{2 k|y| T}{r_{c}}} T_{x^{0} x^{0}}}{g_{00} r_{c}}+\frac{4 k|y| e^{\frac{2 k|y| T}{r_{c}}}\left(T_{x^{0}}\right)^{2}}{g_{00} r_{c} T} \\
& -\frac{6 k^{2}|y|^{2} e^{\frac{2 k|y| T}{r_{c}}}\left(T_{x^{0}}\right)^{2}}{g_{00} r_{c}^{2}}-\frac{g_{33 x^{0}} e^{\frac{2 k|y| T}{r_{c}}} T_{x^{0}}}{g_{00} g_{33} T}-\frac{g_{22 x^{0}} e^{\frac{2 k|y| T}{r_{c}}} T_{x^{0}}}{g_{00} g_{22} T}
\end{aligned}
$$




$$
\begin{aligned}
& -\frac{g_{11 x^{0}} e^{\frac{2 k|y| T}{r_{c}}} T_{x^{0}}}{g_{00} g_{11} T}+\frac{g_{00 x^{0}} e^{\frac{2 k|y| T}{r}} T_{x^{0}}}{g_{00^{2} T} T}+\frac{3 g_{33 x^{0}} k|y| e^{\frac{2 k|y| T}{r_{c}}} T_{x^{0}}}{g_{00} g_{33} r_{c}} \\
& +\frac{3 g_{22 x^{0}} k|y| e^{\frac{2 k|y| T}{r_{c}}} T_{x^{0}}}{g_{00} g_{22} r_{c}}+\frac{3 g_{11 x^{0}} k|y| e^{\frac{2 k|y| T}{r_{c}}} T_{x^{0}}}{g_{00} g_{11} r_{c}}-\frac{3 g_{00 x^{0}} k|y| e^{\frac{2 k|y| T}{r_{c}}} T_{x^{0}}}{g_{00^{2}} r_{c}} \\
& +R_{4} e^{\frac{2 k|y| T}{r_{c}}}+20 k^{2}
\end{aligned}
$$

agora a ação (3.2) junto com $\operatorname{det}\left(g_{M N}\right)=\frac{\sqrt{-g} T}{r_{c}} e^{\frac{-4 k|y| T}{r_{c}}}$ fica

$$
\begin{aligned}
& S=-2 M^{3} \int \frac{\sqrt{-g} T}{r_{c}} e^{\frac{-4 k|y| T}{r_{c}}} d^{4} x d y\left\{-\frac{2 e^{\frac{2 k|y| T}{r_{c}}} T_{x^{3} x^{3}}}{g_{33} T}\right. \\
& +\frac{6 k|y| e^{\frac{2 k|y| T}{r_{c}}} T_{x^{3}} x^{3}}{g_{33} r_{c}}+\frac{4 k|y| e^{\frac{2 k|y| T}{r_{c}}}\left(T_{x^{3}}\right)^{2}}{g_{33} r_{c} T}-\frac{6 k^{2}|y|^{2} e^{\frac{2 k|y| T}{r_{c}}}\left(T_{x^{3}}\right)^{2}}{g_{33} r_{c}^{2}} \\
& +\frac{g_{33 x^{3}} e^{\frac{2 k|y| T}{r_{c}}} T_{x^{3}}}{g_{33^{2}} T}-\frac{g_{22 x^{3}} e^{\frac{2 k|y| T}{r_{c}}} T_{x^{3}}}{g_{22} g_{33} T}-\frac{g_{11 x^{3}} e^{\frac{2 k|y| T}{r_{c}}} T_{x^{3}}}{g_{11} g_{33} T} \\
& -\frac{g_{00 x^{3}} e^{\frac{2 k|y| T}{r_{c}}} T_{x^{3}}}{g_{00} g_{33} T}-\frac{3 g_{33} x^{3} k|y| e^{\frac{2 k|y| T}{r_{c}}} T_{x^{3}}}{g_{33^{2}} r_{c}}+\frac{3 g_{22} x_{x^{3}} k|y| e^{\frac{2 k|y| T}{r_{c}}} T_{x^{3}}}{g_{22} g_{33} r_{c}} \\
& +\frac{3 g_{11 x^{3}} k|y| e^{\frac{2 k|y| T}{r_{c}}} T_{x^{3}}}{g_{11} g_{33} r_{c}}+\frac{3 g_{00 x^{3}} k|y| e^{\frac{2 k|y| T}{r_{c}}} T_{x^{3}}}{g_{00} g_{33} r_{c}}-\frac{2 e^{\frac{2 k|y| T}{r_{c}}} T_{x^{2} x^{2}}}{g_{22} T} \\
& +\frac{6 k|y| e^{\frac{2 k|y| T}{r_{c}}} T_{x^{2} x^{2}}}{g_{22} r_{c}}+\frac{4 k|y| e^{\frac{2 k|y| T}{r_{c}}}\left(T_{x^{2}}\right)^{2}}{g_{22} r T}-\frac{6 k^{2}|y|^{2} e^{\frac{2 k|y| T}{r_{c}}}\left(T_{x^{2}}\right)^{2}}{g_{22} r_{c}^{2}} \\
& -\frac{g_{33 x^{2}} e^{\frac{2 k|y| T}{r_{c}}} T_{x^{2}}}{g_{22} g_{33} T}+\frac{g_{22 x^{2}} e^{\frac{2 k|y| T}{r_{c}}} T_{x^{2}}}{g_{22^{2}}{ }^{2}}-\frac{g_{11 x^{2}} e^{\frac{2 k|y| T}{r_{c}}} T_{x^{2}}}{g_{11} g_{22} T} \\
& -\frac{g_{00 x^{2}} e^{\frac{2 k|y| T}{r_{c}}} T_{x^{2}}}{g_{00} g_{22} T}+\frac{3 g_{33} x^{2} k|y| e^{\frac{2 k|y| T}{r_{c}}} T_{x^{2}}}{g_{22} g_{33} r_{c}}-\frac{3 g_{22 x^{2}} k|y| e^{\frac{2 k|y| T}{r_{c}}} T_{x^{2}}}{g_{22}{ }^{2} r_{c}} \\
& +\frac{3 g_{11 x^{2}} k|y| e^{\frac{2 k|y| T}{r_{c}}} T_{x^{2}}}{g_{11} g_{22} r_{c}}+\frac{3 g_{00 x^{2}} k|y| e^{\frac{2 k|y| T}{r_{c}}} T_{x^{2}}}{g_{00} g_{22} r_{c}}-\frac{2 e^{\frac{2 k|y| T}{r_{c}}} T_{x^{1} x^{1}}}{g_{11} T} \\
& +\frac{6 k|y| e^{\frac{2 k|y| T}{r_{c}}} T_{x^{1} x^{1}}}{g_{11} r_{c}}+\frac{4 k|y| e^{\frac{2 k|y| T}{r_{c}}}\left(T_{x^{1}}\right)^{2}}{g_{11} r_{c} T}-\frac{6 k^{2}|y|^{2} e^{\frac{2 k|y| T}{r_{c}}}\left(T_{x^{1}}\right)^{2}}{g_{11} r_{c}^{2}} \\
& -\frac{g_{33 x^{1}} e^{\frac{2 k|y| T}{r_{c}}} T_{x^{1}}}{g_{11} g_{33} T}-\frac{g_{22 x^{1}} e^{\frac{2 k|y| T}{r_{c}}} T_{x^{1}}}{g_{11} g_{22} T}+\frac{g_{11 x^{1}} e^{\frac{2 k|y| T}{r_{c}}} T_{x^{1}}}{g_{11^{2}} T}
\end{aligned}
$$




$$
\begin{aligned}
& -\frac{g_{00 x_{x^{1}}} e^{\frac{2 k|y| T}{r_{c}}} T_{x^{1}}}{g_{00} g_{11} T}+\frac{3 g_{33 x^{1}} k|y| e^{\frac{2 k|y| T}{r_{c}}} T_{x^{1}}}{g_{11} g_{33} r_{c}}+\frac{3 g_{22 x^{1}} k|y| e^{\frac{2 k|y| T}{r_{c}}} T_{x^{1}}}{g_{11} g_{22} r_{c}} \\
& -\frac{3 g_{11 x^{1}} k|y| e^{\frac{2 k|y| T}{r_{c}}} T_{x^{1}}}{g_{11}{ }^{2} r_{c}}+\frac{3 g_{00 x^{1}} k|y| e^{\frac{2 k|y| T}{r_{c}}} T_{x^{1}}}{g_{00} g_{11} r_{c}}-\frac{2 e^{\frac{2 k|y| T}{r_{c}}} T_{x^{0} x^{0}}}{g_{00} T} \\
& +\frac{6 k|y| e^{\frac{2 k|y| T}{r_{c}}} T_{x^{0} x^{0}}}{g_{00} r_{c}}+\frac{4 k|y| e^{\frac{2 k|y| T}{r_{c}}}\left(T_{x^{0}}\right)^{2}}{g_{00} r_{c} T}-\frac{6 k^{2}|y|^{2} e^{\frac{2 k|y| T}{r_{c}}}\left(T_{x^{0}}\right)^{2}}{g_{00} r_{c}^{2}} \\
& -\frac{g_{33 x^{0}} e^{\frac{2 k|y| T}{r_{c}}} T_{x^{0}}}{g_{00} g_{33} T}-\frac{g_{22 x^{0}} e^{\frac{2 k|y| T}{r_{c}}} T_{x^{0}}}{g_{00} g_{22} T}-\frac{g_{11 x^{0}} e^{\frac{2 k|y| T}{r_{c}}} T_{x^{0}}}{g_{00} g_{11} T} \\
& +\frac{g_{00 x^{0}} e^{\frac{2 k|y| T}{r}} T_{x^{0}}}{g_{00}{ }^{2} T}+\frac{3 g_{33 x^{0}} k|y| e^{\frac{2 k|y| T}{r_{c}}} T_{x^{0}}}{g_{00} g_{33} r_{c}}+\frac{3 g_{22 x^{0}} k|y| e^{\frac{2 k|y| T}{r_{c}}} T_{x^{0}}}{g_{00} g_{22} r_{c}} \\
& \left.+\frac{3 g_{11 x^{0}{ }^{0}} k|y| e^{\frac{2 k|y| T}{r_{c}}} T_{x^{0}}}{g_{00} g_{11} r_{c}}-\frac{3 g_{00 x^{0}} k|y| e^{\frac{2 k|y| T}{r_{c}}} T_{x^{0}}}{g_{00^{2}} r_{c}}+R_{4} e^{\frac{2 k|y| T}{r_{c}}}+20 k^{2}\right\} \text {, }
\end{aligned}
$$

podemos re-escrever os dois primeiros termos dentro da integral de acima como

$$
\begin{aligned}
-\frac{2 \sqrt{-g} e^{\frac{-2 k|y| T}{r_{c}}} T_{x^{3} x^{3}}}{g_{33} r_{c}}= & \partial_{3}\left(-\frac{2 \sqrt{-g} T e^{\frac{-2 k|y| T}{r_{c}}} T_{x^{3}}}{g_{33} r_{c}}\right) \\
& +\frac{\sqrt{-g} T e^{\frac{-4 k|y| T}{r_{c}}}}{r_{c}}\left\{\frac{g_{00 x^{3}} e^{\frac{2 k|y| T}{r_{c}}} T_{x^{3}}}{g_{00} g_{33} T}-\frac{g_{33} x^{3} e^{\frac{2 k|y| T}{r_{c}}} T_{x^{3}}}{g_{33^{2}} T}\right. \\
& +\frac{g_{22 x^{3}} e^{\frac{2 k|y| T}{r_{c}}} T_{x^{3}}}{g_{22} g_{33} T}+\frac{g_{11 x^{3}} e^{\frac{2 k|y| T}{r_{c}}} T_{x^{3}}}{g_{11} g_{33} T} \\
& \left.-\frac{4 k|y| e^{\frac{2 k|y| T}{r_{c}}}\left(T_{x^{3}}\right)^{2}}{g_{33} r_{c} T}\right\}
\end{aligned}
$$


e

$$
\begin{aligned}
\frac{6 \sqrt{-g} T k|y| e^{\frac{-2 k|y| T}{r_{c}}} T_{x^{3} x^{3}}}{g_{33} r_{c}^{2}}= & \partial_{3}\left(\frac{6 \sqrt{-g} T k|y| e^{\frac{-2 k|y| T}{r_{c}}} T_{x^{3}}}{g_{33} r_{c}^{2}}\right) \\
& +\frac{\sqrt{-g} T e^{\frac{-4 k|y| T}{r_{c}}}}{r_{c}}\left\{\frac{12 k^{2}|y|^{2} e^{\frac{2 k|y| T}{r_{c}}}\left(T_{x^{3}}\right)^{2}}{g_{33} r_{c}^{2}}\right. \\
& -\frac{6 k|y| e^{\frac{2 k|y| T}{r_{c}}}\left(T_{x^{3}}\right)^{2}}{g_{33} r_{c}}+\frac{3 g_{33 x^{3}} k|y| e^{\frac{2 k|y| T}{r_{c}}} T_{x^{3}}}{g_{33^{2}} r_{c}} \\
& -\frac{3 g_{22 x^{3}} k|y| e^{\frac{2 k|y| T}{r_{c}}} T_{x^{3}}}{g_{22} g_{33} r_{c}}-\frac{3 g_{11 x^{3}} k|y| e^{\frac{2 k|y| T}{r_{c}}} T_{x^{3}}}{g_{11} g_{33} r_{c}} \\
& \left.-\frac{3 g_{00 x^{3}} k|y| e^{\frac{2 k|y| T}{r_{c}}} T_{x^{3}}}{g_{00} g_{33} r_{c}}\right\}
\end{aligned}
$$

respectivamente.

Analogamente fazemos o mesmo procedimento de integrar por partes aos termos décimo terceiro, décimo quarto, vigésimo quinto, vigésimo sexto, trigésimo sétimo e trigésimo oitavo, depois de substituir as integrais por partes dos termos mencionados, os termos de superfície se anulam na integração quadridimensional e os termos restantes cancelam diversos termos na ação (6), porém com os termos que não cancelam a ação mencionada fica da seguinte forma simplificada

$$
\begin{aligned}
S= & 2 M^{3} \int d^{4} x d y \frac{\sqrt{-g} e^{\frac{-2 k|y| T}{r_{c}}}}{r_{c}}\left\{\frac{6 k|y|}{r_{c}} \partial_{\mu} T \partial^{\mu} T\right. \\
& \left.-\frac{6 k^{2}|y|^{2} T}{r_{c}^{2}} \partial_{\mu} T \partial^{\mu} T-T R_{4}-20 k^{2} T e^{\frac{-2 k|y| T}{r_{c}}}\right\},
\end{aligned}
$$




\section{Referências Bibliográficas}

[1] J. F. Donoghue, E. Golowich and B. Holstein, Dynamics of the Standard Model, D. Reidel Publishing Company, Dordrecht-Hollanf/Ed. Cambridge University Press, 1994

[2] Sidney Coleman, Aspects of Symmetry : Selected Erice Lectures, D. Reidel Publishing Company, Dordrecht-Hollanf/Boston-U.S.A., 2th edition, 1975

[3] M. E. Peskin and D. V. Schroeder, Introduction to Quantum Field Theory, Ed. Addison-Wesley,1995

[4] W. M. Yao and et al., Review of particle physics, Journal of Physics G , 33 (2006).

[5] G. Altarelli, The standard model of particle physics , Encyclopedia of Mathematical Physics, (2005).

[6] LEP, Electroweak Working Group (LEP EWWG)., http://lepewwg.web.cern.ch/LEPEWWG/.

[7] Tevatron, Electroweak Working Group (TEV-EWWG). http://tevewwg.fnal.gov/. 
[8] G. A. Burdman, Theories with extra dimensions. The 15th International Conference on Hadron Collider Physics, 2004, http://arxiv.org/abs/hep$\mathrm{ph} / 0409322 \mathrm{v} 1$.

[9] G. A. Burdman, New Solutions to the Hierarchy Problem. http://arxiv.org/abs/hep-ph/0703194.

[10] Steven Weinberg, The cosmological constant problem, Rev. Mod. Phys. 61, $1-23(1989)$

[11] R. D. Peccei and Helen R. Quinn, Constraints imposed by CP conservation in the presence of pseudoparticles, Physical Review D, 16:1791-1797, 1977.

[12] Th. Kaluza, Sitzungober. Preuss.Akad.Wiss.Berlin, p.966, 1921.

[13] O. Klein, Quantum theory and five dimensional theory of relativity. Zeitschrift für Physik a Hadrons and Nuclei, 37, 12, p. 895-906, 1926.

[14] Lisa Randall e Raman Sundrum. Large Mass Hierarchy from a Small Extra Dimension. Physical Review Letters, 83, 3370-3373, 1999.

[15] A. Pérez-Lorenzana, An introduction to extra dimensions. Journal of Physics: Conference Series, 18, 2005

[16] Tony Gherghetta and Alex Pomarol, Bulk fields and supersymmetry in a slice of AdS , Nuclear Physics B, 586:141-162, 2000.

[17] Gustavo Burdman, Theories with Extra Dimensions, The 15th International Topical Conference on Hadron Collider Physics, East Lansing, Michigan, June 14-18 2004. 
[18] Gustavo Burdman, New Solutions to the Hierarchy Problem, Brazilian Journal of Physics, vol. 37, 2A, June, 2007

[19] N. F. de Sá, Desconstrução Dimensional e Violação de Sabor. Dissertação de Mestrado, Universidade de são Paulo (2011)

[20] Y. Bai, G. Burdman, e C. T. Hill. Topological Interactions in Warped Extra Dimensions. Journal of High Energy Physcis, 2(49), 2010.

[21] J. de Blas, A. Falkowski, M. Pérez-Victoria, e S. Pokorski. Tools for deconstructing gauge theories in $A d S_{5}$., Journal of High Energy Physics, 8(61), 2006.

[22] R. F. Swarttouw. Deconstruction and gauge theories in $A d S_{5}$ Doutorado, Universidade de Tecnologia Delft, 1992.

[23] Christopher T. Hill, Adam K. Leibovich Deconstructing 5D QED Phisical Review D 66, 016006, 2002.

[24] H. J. Rothe. Lattice Gauge Theories. World Scientific, 2005.

[25] G. Burdman, N. Fonseca e L. de Lima, em preparação

[26] Walter D. Goldberger and Mark B. Wise. Modulus Stabilization with Bulk Fields. Physical Review Letters 83, $4922-4925$ (1999).

[27] Walter D. Goldberger and Mark B. Wise. Phenomenology of Stabilized Modulus. Physics Letters B 475 (2000). 275-279.

[28] G. Burdman, Victor M. P. Cano, A Dilaton in Full-hierarchy Quiver Theories, em preparação 
[29] Csaba Csáki, Jay Hubisz, and Seung J. Lee. Radion phenomenology in realistic warped space models. PHYSICAL REVIEW D 76, 125015 (2007). 